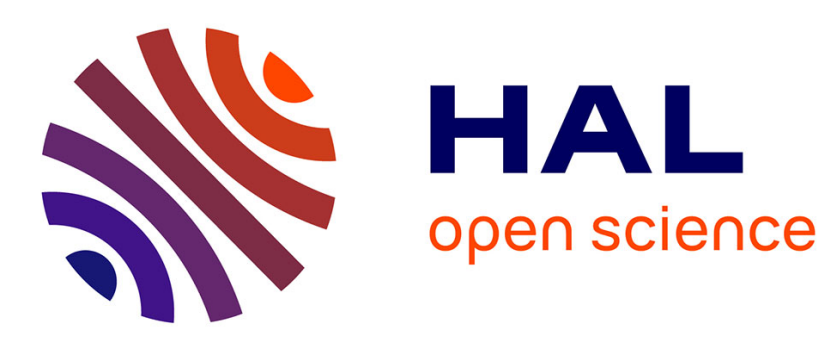

\title{
Nonconvex model of material growth. Mathematical theory
}

Jean-François Ganghoffer, Pavel Igorevich Plotnikov, Jan Sokolowski

\section{To cite this version:}

Jean-François Ganghoffer, Pavel Igorevich Plotnikov, Jan Sokolowski. Nonconvex model of material growth. Mathematical theory. 2015. hal-01203772

\section{HAL Id: hal-01203772 \\ https://hal.science/hal-01203772}

Preprint submitted on 1 Oct 2015

HAL is a multi-disciplinary open access archive for the deposit and dissemination of scientific research documents, whether they are published or not. The documents may come from teaching and research institutions in France or abroad, or from public or private research centers.
L'archive ouverte pluridisciplinaire HAL, est destinée au dépôt et à la diffusion de documents scientifiques de niveau recherche, publiés ou non, émanant des établissements d'enseignement et de recherche français ou étrangers, des laboratoires publics ou privés. 
Noname manuscript No.

(will be inserted by the editor)

J.F. Ganghoffer \& P.I. Plotnikov \& J.

Sokolowski

\title{
Nonconvex Model of Material Growth. Mathematical Theory
}

\begin{abstract}
The model of volumetric material growth is introduced in the framework of finite elasticity. The new results obtained for the model are presented with complete proofs. The state variables include the deformations, temperature and the growth factor matrix function. The existence of global in time solutions for the quasistatic deformations boundary value problem coupled with the energy balance and the evolution of the growth factor is shown. The mathematical results can be applied to a wide class of growth models in mechanics and biology.
\end{abstract}

Key words: Volumetric growth, thermoelasticity, evolutionary problem, nonconvexity.

AMS: 76B15; 47J15; 35S15; 76B07

J.F. Ganghoffer

LEMTA, Nancy Université, 2, Avenue de la Forêt de Haye, BP 160, TSA 60604, 54518 Vandoeuvre Cedex, France

Tel.: +33-3-83595724

Fax: +33-3-83595551

E-mail: Jean-Francois.Ganghoffer@univ-lorraine.fr

P.I. Plotnikov

Novosibirsk State University, Pirogova 2, 630090 Novosibirsk, Russia

Tel.: +7-383-3333123

Fax: +7-383-3331612

E-mail: plotnikov@hydro.nsc.ru

J. Sokolowski

Institut Elie Cartan Nancy, UMR7502 (Université Lorraine, CNRS, INRIA), Laboratoire de Mathématiques, Université de Lorraine, B.P.239, 54506 Vandoeuvre-lèsNancy Cedex, France

Tel.: +33-3-83 684580

Fax: +33-3-83 684534

E-mail: Jan.Sokolowski@univ-lorraine.fr 


\section{Introduction}

Growth (resp. atrophy) refers to physical processes in which the material of a solid body increases (resp. decreases) its size by addition (resp. removal) of mass. The advantages and drawbacks of the existing growth models are discussed in the recent papers [16] and [18]. A first class of such models are kinematic models describing the evolution of the material growth towards a homeostatic state, rely on the kinematic decomposition of the transformation gradient into a generally incompatible mapping and an elastic mapping; they were historically introduced in [22] and developed in [26, 23, 1,27]. Approaches analogous to elastoplasticity were then developed in a rational framework basing on the second principle of thermodynamics for open systems, in order to identify the evolution laws of growth $[7,17,19,20]$. It is important to note the prominent role of Eshelby stress in relation to the material driving forces for growth $[5,7,13,14]$, The mathematical aspects of volumetric growth models are poorly investigated. The local existence and uniqueness results were established in $[11,12]$.

Mechanical background. Thermoelastic material. In this paragraph we briefly discuss basic facts from finite elasticity theory. Throughout, we shall assume that $\Omega \subset \mathbb{R}^{3}$ is a bounded reference domain with boundary $\partial \Omega$ of class $C^{\infty}$ in the space of variable $x$. The state of an elastic material is characterized by a deformation field $\mathbf{u}=\left(u_{1}, u_{2}, u_{3}\right): \Omega \times[0, T] \rightarrow \mathbb{R}^{3}$ and the Kelvin temperature $\theta: \Omega \times[0, T] \rightarrow \mathbb{R}^{+}$. The elastic distorsion tensor $D \mathbf{u}$ is the Jacobi matrix of the mapping $\mathbf{u}$ with entries

$$
(D \mathbf{u})_{i j}(x, t)=\partial_{x_{j}} u_{i}(x, t), \quad(x, t) \in \Omega \times[0, T] .
$$

The second derivative (Hessian) of the displacement field is the third order tensor $D^{2} \mathbf{u}$ with entries

$$
\left(D^{2} \mathbf{u}\right)_{i j k}(x, t)=\partial_{x_{j}} \partial_{x_{k}} u_{i}(x, t), \quad(x, t) \in \Omega \times[0, T] .
$$

We will assume that the material is hyperelastic and its properties are described by a specific free energy density $\Psi\left(D^{2} \mathbf{u}, D \mathbf{u}, \theta\right)$. In this case the entropy $v\left(D^{2} \mathbf{u}, D \mathbf{u}, \theta\right)$ and internal energy $e\left(D^{2} \mathbf{u}, D \mathbf{u}, \theta\right)$ are defined by

$$
v=-\partial_{\theta} \Psi\left(D^{2} \mathbf{u}, D \mathbf{u}, \theta\right), \quad e=\Psi\left(D^{2} \mathbf{u}, D \mathbf{u}, \theta\right)-\theta \partial_{\theta} \Psi\left(D^{2} \mathbf{u}, D \mathbf{u}, \theta\right)
$$

i.e., $e=\Psi+v \theta$. It is important to note that the density of the Helmholtz free energy is a concave function of the temperature. In many applications the dependence of the free energy on $D^{2} \mathbf{u}$ is quadratic. In particular, for the $3 \mathrm{D}$ Falk model which is widely accepted in the theory of solid-solid phase transitions (see $[10,21,25]$ ), the the free energy density is

$$
\Psi=\frac{\varepsilon}{2}|\Delta \mathbf{u}|^{2}+\theta W(D \mathbf{u})+c_{1} \theta-c_{2} \theta \log \theta .
$$

Notice that in this case the free energy density has a weak singularity at $\theta=0$. A further simplification is the linearization of problem with respect to temperature near some average value $\theta_{c}$. Without loss of generality we 
can assume that $\theta_{c}=1$. This means that $\theta=1+\vartheta$ and all terms of order $O\left(\vartheta^{3}\right)$ are neglected. Thus we get the following expression for the free energy density:

$$
\Psi=\frac{\varepsilon}{2}|\Delta \mathbf{u}|^{2}+(1+\vartheta) W(D \mathbf{u})-\frac{1}{2} \vartheta^{2} .
$$

Here we take for simplicity $c_{1}=c_{2}=1$. If the free energy is in the form (1.2), then we have the following expression for the entropy $v$, the internal energy $e$, the second order Piola-Kirchhoff stress tensor $\mathbf{T}_{f}$, and the third order Piola-Kirchhoff hyperstress tensor $\mathbf{T}_{s}$ :

$$
\begin{array}{r}
v=\vartheta-W(\mathbf{D} u), \quad e=\frac{\varepsilon}{2}|\Delta \mathbf{u}|^{2}+W(D \mathbf{u})+\frac{1}{2} \vartheta^{2}, \\
\mathbf{T}_{f}=(1+\vartheta) W^{\prime}(D \mathbf{u}), \quad\left(T_{s}\right)_{i j k}=\varepsilon \Delta u_{i} \delta_{j k} .
\end{array}
$$

Here the notation $W^{\prime}(\boldsymbol{\xi})$ stands for the matrix with entries

$$
\left(W^{\prime}(\boldsymbol{\xi})\right)_{i j}=\partial_{\xi_{i j}} W(\boldsymbol{\xi})
$$

$\delta_{j k}$ is the Kronecker delta. The problem consists in finding a displacement field $\mathbf{u}$ and a temperature $\vartheta$ satisfying the momentum balance equation and the energy balance equation

$$
\frac{\partial e}{\partial t}+\operatorname{div} \mathbf{q}=\mathbf{T}_{f}: \frac{\partial D \mathbf{u}}{\partial t}+\mathbf{T}_{s}: \frac{\partial D^{2} \mathbf{u}}{\partial t}+g
$$

Here $g$ is an exterior heat source. In view of the Fourier law we can take $\mathbf{q}=-\nabla \vartheta$. The energy balance equation can be rewritten in the equivalent form

$$
\partial_{t} v=-\theta^{-1} \operatorname{div} \mathbf{q}+\theta^{-1} g .
$$

Linearization of this equation near the equilibrium temperature $\theta=1$ leads to the equation

$$
\partial_{t} v=\Delta \vartheta+g
$$

Mechanical backgrounds. Growing material. The main axiom of the theory of volumetric growth for elastic materials is the hypothesis that the distorsion tensor admits the representation

$$
D \mathbf{u}=\mathbf{F}_{e} \mathbf{F}_{g}
$$

where the growth factor (implant) $\mathbf{F}_{g}$ is responsible for the material growth and $\mathbf{F}_{e}$ corresponds to the elastic deformations. In the important particular case of isotropic growth the growth factor has the representation $\mathbf{F}_{g}=w \mathbf{I}$ where $w(x, t)$ is a scalar. Notice that the multiplicative decomposition of the distorsion tensor is often used in finite deformation continuum mechanics as a basis for treating mechanical effects including plasticity, biological growth, material morphogenesis, and dislocations [6].

The second assumption of volumetric growth theory is that the Helmholtz free energy $\Psi_{g}$ of the growing material has the form

$$
\begin{aligned}
\Psi_{g}\left(D^{2} \mathbf{u}, D \mathbf{u}, \vartheta, \mathbf{F}_{g}\right):=\operatorname{det} \mathbf{F}_{g} \Psi\left(\mathbf{Q}_{e}, D \mathbf{u} \mathbf{F}_{g}^{-1}, \vartheta\right) \\
\\
\quad \equiv \operatorname{det} \mathbf{F}_{g} \Psi\left(\mathbf{Q}_{e}, \mathbf{F}_{e}, \vartheta\right)
\end{aligned}
$$


Here $\Psi$ is the basic Helmholtz free energy of the original thermoelastic material, and $\mathbf{Q}_{e}$ is the third order tensor with components

$$
\left(\mathbf{Q}_{e}\right)_{i j k}=\partial_{x_{\alpha}} \partial_{x_{\beta}} u_{i}\left(\mathbf{F}_{g}^{-1}\right)_{\alpha j}\left(\mathbf{F}_{g}^{-1}\right)_{\beta k}
$$

In the isotropic case with $\mathbf{F}_{g}=w \mathbf{I}$ we have $\mathbf{Q}_{e}=w^{-2} D^{2} \mathbf{u}$. The entropy of the growing material is defined by the relation

$$
v_{g}=-\operatorname{det} \mathbf{F}_{g} \frac{\partial}{\partial \vartheta} \Psi\left(\mathbf{Q}_{e}, D \mathbf{u} \mathbf{F}_{g}^{-1}, \vartheta\right)
$$

As shown in $[5,7]$, the Clausius-Duhem inequality and the principle of independence of motions imply the following expressions for the stress tensors:

$$
\begin{gathered}
\left(\mathbf{T}_{f}\right)_{i j}=\operatorname{det} \mathbf{F}_{g} \frac{\partial}{\partial(D \mathbf{u})_{i j}} \Psi\left(\mathbf{Q}_{e}, D \mathbf{u} \mathbf{F}_{g}^{-1}, \vartheta\right), \\
\left(\mathbf{T}_{s}\right)_{i j k}=\operatorname{det} \mathbf{F}_{g} \frac{\partial}{\partial\left(D^{2} \mathbf{u}\right)_{i j k}} \Psi\left(\mathbf{Q}_{e}, D \mathbf{u} \mathbf{F}_{g}^{-1}, \vartheta\right) .
\end{gathered}
$$

Moreover, the Clausius-Duhem inequality yields the relation

$$
\mathbf{b}:\left(\partial_{t} \mathbf{F}_{g} \mathbf{F}_{g}^{-1}\right) \leq 0,
$$

where $\mathbf{b}$ is the material Eshelby tensor (cf. [8]) given by

$$
\mathbf{b}=\Psi_{g} \mathbf{I}-\mathbf{F}_{e}^{\top} \frac{\partial \Psi_{g}}{\partial \mathbf{F}_{e}}-2\left\{\frac{\partial \Psi_{g}}{\partial \mathbf{Q}_{e}}: \mathbf{Q}_{e}\right\}^{\top}
$$

In particular, for the isotropic growth we have the following expressions for the Helmholtz free energy density and the entropy:

$$
\begin{aligned}
\Psi_{g}\left(D^{2} \mathbf{u}, D \mathbf{u}, \vartheta, w\right) & =\frac{\varepsilon}{2 w}|\Delta \mathbf{u}|^{2}+(1+\vartheta) w^{3} W\left(w^{-1} D \mathbf{u}\right)-\frac{w^{3} \vartheta^{2}}{2} \\
v_{g} & =w^{3} v, \text { where } v=\vartheta-W\left(w^{-1} D \mathbf{u}\right) .
\end{aligned}
$$

In this case the stress tensors are defined by the relations

$$
\mathbf{T}_{f}=(1+\vartheta) w^{2} W^{\prime}\left(w^{-1} D \mathbf{u}\right), \quad\left(T_{s}\right)_{i j k}=\varepsilon w^{-1} \Delta u_{i} \delta_{j k} .
$$

The total Helmholtz free energy is given by

$$
\boldsymbol{\Psi}_{g}(\mathbf{u}, \vartheta, w)=\int_{\Omega} \Psi_{g}\left(D^{2} \mathbf{u}, D \mathbf{u}, \vartheta, w\right) d x
$$

The system of the governing equations includes the momentum balance equation and the entropy balance equation. Since the characteristic time of growth of biological materials is about days or weeks, we can neglect the inertial forces and take the momentum balance equation in the quasi static form

$$
\operatorname{div}\left(\mathbf{T}_{f}-\operatorname{div} \mathbf{T}_{s}\right)+\mathbf{f}=0,
$$


where $\mathbf{f}$ is a given exterior force. For simplicity we discard the exterior heat source and take the linearized entropy balance equation in the form

$$
\partial_{t} v_{g}=\Delta \vartheta
$$

In order to obtain a closed system of equations for the displacement field $\mathbf{u}$, the temperature $\vartheta$, and the growth factor $\mathbf{F}_{g}$, we need an extra equation for $\mathbf{F}_{g}$. We assume that the evolution of the growth factor is described by a nonconservative model. This model is based on the assumption that $\partial_{t} \mathbf{F}_{g}$ is a function of the deformation gradient, the temperature and the growth factor. The specification of such a function is the most important question of the theory. As noted in $[5,7]$, the Clausius-Duhem inequality (1.11) and the covariance principle lead to the following evolution equation for the implant $\mathbf{F}_{g}$ :

$$
\partial_{t} \mathbf{F}_{g} \mathbf{F}_{g}^{-1}=-c_{0}^{+} \operatorname{tr} \mathbf{b} \mathbf{I}-c_{1}^{+} \mathbf{b},
$$

where $c_{k}^{+}=c_{k}^{+}\left(I_{i}, \vartheta\right)$ are nonnegative functions of the temperature and the invariants $I_{i}$ of the Eshelby tensor. Notice that the right hand side of (1.17) is the only tensor function satisfying (1.11). We restrict our considerations to the case of the isotropic growth with $\mathbf{F}_{g}=w \mathbf{I}$. In this case we have

$$
3 w=\operatorname{tr} \mathbf{F}_{g}=I_{1}, I_{2}=3 w^{2}, I_{3}=w^{3}, c_{1}^{+}=0 .
$$

Hence we can rewrite equation (1.17) in the form

$$
3 \partial_{t} w=-w c_{0}^{+}(\vartheta, \operatorname{tr} \mathbf{b}) \operatorname{tr} \mathbf{b} .
$$

It follows that the simplest version of the evolution equation for the growth factor is the ordinary differential equation

$$
\partial_{t} w=-w H(\operatorname{tr} \mathbf{b})
$$

where $H: \mathbb{R} \rightarrow \mathbb{R}$ is a smooth function such that $H(s) s \geq 0$.

Problem formulation. Results Combining momentum equation (1.13c)-(1.15) and entropy balance equation (1.16) with the evolution equation (1.19) we arrive at the following system of differential equations which describe the isotropic volumetric growth of the thermoelastic material:

$$
\begin{gathered}
\varepsilon \Delta\left(w^{-1} \Delta \mathbf{u}\right)-\operatorname{div}\left(w^{2}(1+\vartheta) W^{\prime}\left(w^{-1} D \mathbf{u}\right)\right)=\mathbf{f} \text { in } \Omega \times(0, T) \\
\partial_{t}\left(w^{3} v\right)=\Delta \vartheta \text { in } \Omega \times(0, T), \\
\partial_{t} w=-w H(\varphi) \text { in } \Omega \times(0, T) .
\end{gathered}
$$

Here the temperature $\vartheta$ is given by

$$
\vartheta=v+W\left(w^{-1} D \mathbf{u}\right) .
$$

Formulae (1.12) and (1.13a) imply the following expression for the trace of the Eshelby tensor $\varphi:=\operatorname{tr} \mathbf{b}$ :

$$
\begin{array}{r}
\varphi=-\frac{\varepsilon}{2 w}|\Delta \mathbf{u}|^{2}+(1+\vartheta) w^{3}\left(3 W\left(w^{-1} D \mathbf{u}\right)-\right. \\
\left.W^{\prime}\left(w^{-1} D \mathbf{u}\right):\left(w^{-1} D \mathbf{u}\right)\right)-\frac{3 w^{3}}{2} \vartheta^{2} .
\end{array}
$$


Equations (1.20a)-(1.20c) should be supplemented with boundary and initial conditions. We take them in the form

$$
\begin{gathered}
\mathbf{u}=\mathbf{h}, \quad \Delta \mathbf{u}=0, \quad \frac{\partial \vartheta}{\partial n}+\vartheta=0 \quad \text { on } \partial \Omega \times(0, T), \\
\left.v\right|_{t=0}=v_{0},\left.\quad w\right|_{t=0}=w_{0} \text { in } \Omega,
\end{gathered}
$$

where $n$ is the outward normal to $\partial \Omega$. The boundary condition for $\vartheta$ is the standard radiation condition. The boundary condition for the displacement means that the growing material is surrounded by the duct membrane whose shape is defined by the function $\mathbf{h}$. The equations and the boundary and initial conditions (1.20) form a closed boundary value problem for the deformation field $\mathbf{u}$, entropy $v$, temperature $\vartheta$, and the growth factor $w$. Further we assume that the stored potential energy $W$, the function $H$, and the boundary data satisfy the following conditions.

H.1 The function $W$ is in $C^{\infty}\left(\mathbb{R}^{9}\right)$ and there is $\kappa \in[2,3)$ such that

$$
0 \leq W(\boldsymbol{\xi}) \leq c(1+|\boldsymbol{\xi}|)^{\kappa},\left|W^{\prime}(\boldsymbol{\xi})\right| \leq c(1+|\boldsymbol{\xi}|)^{\kappa-1},\left|W^{\prime \prime}(\boldsymbol{\xi})\right| \leq c(1+|\boldsymbol{\xi}|)^{\kappa-2} .
$$

H.2 The function $H \in C^{\infty}(\mathbb{R})$ satisfies the conditions

$$
H^{\prime}(s) \geq 0, \quad H(0)=0, \quad\left|H^{\prime}(\varphi)\right| \leq c, \quad\left|H^{\prime}(\varphi)\right| \leq c(1+|\varphi|)^{-1} .
$$

H.3 For simplicity we assume that $\partial_{t} \mathbf{h}=0, \partial_{t} \mathbf{f}=0$. We also assume that the given data satisfy the conditions

$$
\begin{gathered}
v_{0} \in W^{1,2}(\Omega), \quad \mathbf{f} \in L^{\infty}(\Omega), \quad \mathbf{h} \in C^{4}(\Omega), \\
w_{0} \in W^{1,2}(\Omega), \quad 0<c^{-1}<w_{0}<c<\infty .
\end{gathered}
$$

We are looking for a weak solution to problem (1.20), which is defined as follows.

Definition 1.1 (Weak formulation) Denote by $\mathcal{W}^{2, p}, 1<p<\infty$, the Banach space which consists of all functions $\mathbf{u} \in W_{0}^{1, p}(\Omega)$ such that

$$
\|\mathbf{u}\|_{\mathcal{W}^{2, p}}=\left(\int_{\Omega}|\Delta \mathbf{u}|^{p} d x\right)^{1 / p}<\infty, \quad \mathbf{u}=0 \quad \text { on } \partial \Omega .
$$

The space $\mathcal{W}^{2, p}$ is topologically and algebraically isomorphic to the space $W^{2, p}(\Omega) \cap W_{0}^{1, p}(\Omega)$. A tuple of functions $(\mathbf{u}, v, w, \varphi)$ is said to be a weak solution to problem (1.20) if

(i) For a.e. $t \in(0, T)$ the function $\mathbf{u}(t)-\mathbf{h}$ belongs to the class $\mathcal{W}^{2,2} \cap$ $W^{2,6}(\Omega)$,

$$
\begin{array}{r}
v, w, \vartheta \in L^{2}\left(0, T ; W^{1,2}(\Omega)\right), \quad w^{ \pm 1} \in L^{\infty}(\Omega \times(0, T)) \\
v, \vartheta \in L^{\infty}\left(0, T ; L^{2}(\Omega)\right) .
\end{array}
$$

(ii) The function $w$ satisfies equation (1.20c) and initial condition (1.20g). The temperature $\vartheta$ and the trace of the Eshelby tensor $\varphi$ are connected with the growth factor $w$ and the entropy $v$ by the relation $(1.20 \mathrm{~d})$. 
(iii) The integral identity

$$
\begin{aligned}
\int_{0}^{T} \int_{\Omega}\left(w^{3} v \partial_{t} \varsigma-\nabla \vartheta \cdot \nabla \varsigma\right) & d x d t \\
& -\int_{\partial \Omega} \vartheta \varsigma d s+\int_{\Omega} w_{0}^{3} v_{0} \varsigma(x, 0) d x=0
\end{aligned}
$$

holds for all $\varsigma \in C^{\infty}(\Omega \times(0, T))$ vanishing on $\Omega \times\{t=T\}$.

(iv) The integral identity

$$
\begin{aligned}
\int_{\Omega}\left(\frac{\varepsilon}{w} \Delta \mathbf{u}(t)\right. & \cdot \Delta \boldsymbol{\xi} \\
& \left.+(1+\vartheta(t)) W^{\prime}\left(w^{-1}(t) D \mathbf{u}(t)\right): D \boldsymbol{\xi}-f \cdot \boldsymbol{\xi}\right) d x=0
\end{aligned}
$$

holds for all $\boldsymbol{\xi} \in C^{2}(\Omega)$ vanishing on $\partial \Omega$ and for almost all $t \in(0, T)$.

Definition 1.1 does not determine a solution to problem (1.20) in a unique way. Notice that there is a disparity between the unknown functions in equations (1.20). These equations involve time derivatives of the entropy $v$ and of the growth factor $w$, i.e., $v$ and $w$ are evolutionary variables. The displacement vector field satisfies static equation (1.20a). The properties of solutions to this equations are completely determined by the stored elastic energy density $W$. In nonlinear elasticity, $W$ is polyconvex but it is not convex, see [3] for discussion. Moreover, if the free energy density is in the form (1.2), then it is not convex and it is not bounded from below even if $W$ is convex. It follows that in the general case the momentum balance equations represented by the integral identity (1.26) has multiple solutions. Hence, for given $v$ and $w$ there are many temperatures $\vartheta$ and traces $\varphi$ satisfying relations (1.20d)$(1.20 \mathrm{e})$, and the number of these quantities depends on the time variable. In order to diminish this arbitrariness, it is necessary to supplement equations and boundary conditions (1.20) with additional selection rules. We intend to prove that such rules can be formulated as follows. Introduce the functions

$$
\Theta(D \mathbf{u}, v, w)=v+W\left(w^{-1} D \mathbf{u}\right), \quad V(D \mathbf{u}, \vartheta, w)=\vartheta-W\left(w^{-1} D \mathbf{u}\right) .
$$

Denote by $E=\Psi_{g}+V \vartheta w^{3}$ the density of the internal energy as a function of the temperature and growth factor, and denote by $\mathcal{E}$ the density of the internal energy as a function of the entropy and growth factor. Calculations show that

$$
\begin{aligned}
& E=\frac{\varepsilon}{2 w}|\Delta \mathbf{u}|^{2}+w^{3} W\left(w^{-1} D \mathbf{u}\right)+w^{3} \frac{\vartheta^{2}}{2} \\
& \mathcal{E}=\frac{\varepsilon}{2 w}|\Delta \mathbf{u}|^{2}+w^{3} W\left(w^{-1} D \mathbf{u}\right)+\frac{1}{2} w^{3} \Theta(D \mathbf{u}, v, w)^{2} .
\end{aligned}
$$

We denote by $\mathbf{E}$ the total internal energy as a function of the displacements, temperature, and growth factor, and denote by $\mathcal{E}$ the total internal energy 
as a function of the displacements, entropy, and growth factor, i.e.,

$\mathbf{E}(\mathbf{u}, \vartheta, w)=\int_{\Omega} E\left(D^{2} \mathbf{u}, D \mathbf{u}, \vartheta, w\right) d x, \mathcal{E}(\mathbf{u}, v, w)=\int_{\Omega} \mathcal{E}\left(D^{2} \mathbf{u}, D \mathbf{u}, v, w\right) d x$.

Definition 1.2 (Work and marginal function) Introduce the functional

$$
\mathcal{H}(\mathbf{u}, v, w)=\mathcal{E}(\mathbf{u}, v, w)-\int_{\Omega} \mathbf{f} \cdot \mathbf{u} d x .
$$

We define the marginal function $\mathbf{M}$ of the functional $\mathcal{H}$ by the relation

$$
\mathbf{M}(v, w)=\inf _{\mathbf{u}-\mathbf{h} \in \mathcal{W}^{2,2}} \mathcal{H}(\mathbf{u}, v, w, f) .
$$

Notice that $\mathbf{M}(v, w)$ is well defined if $v \in L^{2}(\Omega), \mathbf{f} \in L^{\infty}(\Omega)$, and $w^{ \pm 1} \in$ $L^{\infty}(\Omega)$.

Definition 1.3 (Selection principle 1) Suppose a weak solution to problem (1.20) satisfies all conditions of Definition 1.1. We say that the displacement field $\mathbf{u}$ satisfies the first selection principle if $\mathcal{H}(\mathbf{u}(t), v(t), w(t))=$ $\mathbf{M}(v(t), w(t))$ for a.e. $t \in(0, T)$. In other words the displacement field $\mathbf{u}(t)$ is a minimizer of the functional $\mathcal{H}(\cdot, v(t), w(t))$.

Denote by $\Pi$ the total dissipation rate

$$
\Pi(\vartheta, \varphi)=\int_{\Omega}\left(|\nabla \vartheta|^{2}+H(\varphi) \varphi\right) d x+\int_{\partial \Omega} \vartheta^{2} d s
$$

It is convenient to represent $\Pi$ as the sum of two forms

$$
\begin{gathered}
\Pi=\Pi_{0}(\vartheta, \vartheta)+\Pi_{1}(H(\varphi), \varphi) \\
\Pi_{0}(\vartheta, v)=\int_{\Omega} \nabla \vartheta \nabla v d x+\int_{\partial \Omega} \vartheta v d s, \quad \Pi_{1}(\varphi, \psi)=\int_{\Omega} \psi \varphi d x
\end{gathered}
$$

Definition 1.4 (Admissible set) For given $v \in W^{1,2}(\Omega)$ and strictly positive $w \in L^{\infty}(\Omega)$, denote by $\mathcal{P}(v, w)$ the set of all couples $(\vartheta, \varphi)$ with the following property: There is $\mathbf{u} \in \mathcal{W}^{2,2}+\mathbf{h}$ such that

$$
\vartheta=v+W\left(w^{-1} D \mathbf{u}\right), \varphi=\varphi\left(D^{2} \mathbf{u}, D \mathbf{u}, \vartheta, w\right), \quad \mathcal{H}(\mathbf{u}, v, w)=\mathbf{M}(v, w) .
$$

Definition 1.5 (Selection principle 2) Suppose a weak solution to problem (1.20) satisfies all conditions of Definition 1.1. We say that the functions $\vartheta$ and $\varphi$ satisfy the second selection principle if the inequality

$$
\Pi(\vartheta(t), \varphi(t)) \leq \Pi_{0}(\tilde{\vartheta}, \vartheta(t))+\Pi_{1}(H(\varphi(t)), \tilde{\varphi}) .
$$

holds for every $(\tilde{\vartheta}, \tilde{\varphi}) \in \mathcal{P}(v(t), w(t))$ and for a.e. $t \in(0, T)$.

The following theorem is the main result of this paper.

Theorem 1.1 Under the above assumptions, problem (1.20) has a weak solution which meets all requirements of Definition 1.1. For a. e.t and $v=v(t)$, $w=w(t)$, the functions $\mathbf{u}(t), \vartheta(t)$, and $\varphi(t)$ satisfy the selection principles given by Definitions 1.3 and 1.5 . 
Mathematical background. The proof of Theorem 1.1 is based on compactness and monotonicity arguments. There are three aspects of our method which deserve brief mention. The first is the implicit time discretization scheme for problem (1.20). Using this scheme we construct approximate solutions to this problem as saddle points of the "action" functional. The second aspect is the formulation of monotonicity inequalities for the sequence of the approximation solutions in terms of the marginal function. These inequalities allow us to eliminate the displacements from the further analysis and, by doing so, cope with the nonconvexity of the free energy with respect to the displacement field. The third aspect is the systematic application of the theory of sliced measures in Banach spaces to the problem of compactness of approximate solutions.

We now explain the organization of the paper. In Section 2 we employ the time discretization scheme in order to construct a sequence of approximate solutions $\mathbf{u}_{N}, v_{N}, w_{N}, \vartheta_{N}$, and $\varphi_{N}$ to problem (1.20). We deduce estimates for the approximate solutions. In particular, we show that the $\mathbf{u}_{N}$ are bounded in the space $L^{\infty}\left(0, T ; W^{2,6}(\Omega)\right)$ and that the strictly positive functions $w_{N}$ are uniformly bounded from below and above. We also prove that the sequences $\vartheta_{N}$ and $\varphi_{N}$ are bounded in the Lebesgue spaces $L^{r}\left(0, T ; L^{p}(\Omega)\right)$ and $L^{s}\left(0, T ; L^{q}(\Omega)\right)$ for all exponents satisfying inequalities $(2.20)$.

In Section 3 we investigate the compactness properties of the approximate solutions. We show that the sequences $v_{N}$, and $w_{N}$ contain subsequences, still denoted by $v_{N}$ and $w_{N}$, such that $v_{N}$ converges to some $v$ in $L^{r}\left(0, T ; L^{p}(\Omega)\right)$ and $w_{N}$ converges to some $w$ a.e. in $\Omega \times(0, T)$. Moreover, in Section 3 we show that for every $\eta>0$ there is a compact set $\mathcal{T}_{\eta} \subset(0, T)$ with meas $\left((0, T) \backslash \mathcal{T}_{\eta}\right)<$ $\eta$ such that the totality of the functions $\left(\vartheta_{N}(t), \varphi_{N}(t)\right)$ belongs to a compact set $\Sigma_{\eta} \subset L^{p}(\Omega) \times L^{q}(\Omega)$.

Sections 4 and 5 are the heart of the paper. In Section 4 we derive the monotonicity relations. We prove that for a.e. $0<t_{1}<t_{0}<T$, the approximate solutions satisfy the energy dissipation inequality

$$
\begin{aligned}
& \mathbf{M}\left(v\left(t_{0}\right), w\left(t_{0}\right)\right)-\mathbf{M}\left(v\left(t_{1}\right), w\left(t_{1}\right)\right)+\limsup _{N \rightarrow \infty}\left\{\int_{t_{1}+T / N}^{t_{0}} \Pi_{1}\left(H\left(\varphi_{N}\right), \varphi_{N}\right)\right. \\
& \left.+\frac{1}{2} \int_{t_{1}+T / N}^{t_{0}} \Pi_{0}\left(\vartheta_{N}, \vartheta_{N}\right) d s+\frac{1}{2} \int_{t_{1}+T / N}^{t_{0}-T / N} \Pi\left(\bar{\vartheta}_{N}, \bar{\vartheta}_{N}\right) d s\right\} \leq 0
\end{aligned}
$$

where the auxiliary functions $\bar{\vartheta}_{N}$ satisfy the conditions $\bar{\vartheta}_{N}-\vartheta_{N} \rightarrow 0$ in $L^{2}\left(0, T ; L^{2}(\Omega)\right)$ as $N \rightarrow \infty$. We also prove that the complementary inequality

$$
\begin{aligned}
\liminf _{t_{1} \nearrow t_{0}} \frac{1}{t_{0}-t_{1}}\left\{\mathbf{M}\left(v\left(t_{0}\right), w\left(t_{0}\right)\right)-\mathbf{M}\left(v\left(t_{1}\right), w\left(t_{1}\right)\right)\right\} \\
+\Pi_{0}\left(\vartheta, \vartheta^{*}\left(t_{0}\right)\right)+\Pi_{1}\left(\varphi, H^{*}\left(t_{0}\right)\right) \geq 0
\end{aligned}
$$

holds true for every $(\vartheta, \varphi) \in \mathcal{P}\left(v\left(t_{0}\right), w\left(t_{0}\right)\right)$. Here $\vartheta^{*}, H^{*}$ are weak limits of the sequences $\vartheta_{N}$ and $H\left(\varphi_{N}\right)$. Notice that the monotonicity relations (1.36) and (1.37) do not involve the displacement field. In Section 5 we obtain a representation for the weak limits of the sequences $\left(\vartheta_{N}, \varphi_{N}\right)$. We prove the 
existence of a measurable family of probability measures $\mu_{t}$ on the compact set $\Sigma_{\eta} \subset L^{p}(\Omega) \times L^{q}(\Omega)$ such that

$$
\lim _{N \rightarrow \infty} \int_{\mathcal{T}_{\eta}} \int_{\Omega} F\left(t, \vartheta_{N}, \varphi_{N}\right) d x d t=\int_{\mathcal{T}_{\eta}}\left\{\int_{\Sigma_{\eta}} F(t, \vartheta, \varphi) d \mu_{t}(\vartheta, \varphi)\right\} d t
$$

for every continuous function $F:[0, T] \times \Sigma_{\eta} \rightarrow \mathbb{R}$. Here $\mathcal{T}_{\eta}$ is a compact set such that meas $(0, T) \backslash \mathcal{T}_{\eta} \leq \eta$, where $\eta$ is an arbitrary positive number. Representation (1.38) has some advantages over the standard representation of weak limits via the Young measure, since $F$ in (1.38) is a general nonlinear functional. It may be an integro-differential form like $\Pi$ or a nonlinear integral operator. It is a remarkable fact that the support of $\mu_{t}$ is contained in the set $\mathcal{P}(v(t), w(t))$ given by Definition 1.4. This means that for $\mu_{t}$-almost every $(\vartheta, \varphi)$ there is a displacement field $\mathbf{u}$ such that $\mathbf{u}, \vartheta$, and $\varphi$ satisfy relations (1.34).

In Section 6 we use inequalities (1.36)-(1.37) and representation (1.38) in order to prove that $\mu_{t}$ is the Dirac measure concentrated at the point $\left(\vartheta^{*}, \varphi^{*}\right) \in L^{p}(\Omega) \times L^{q}(\Omega)$. This result yields the strong convergence of the sequences $\vartheta_{N}$ and $\varphi_{N}$. In Section 7 we prove that the limits $\vartheta^{*}, \varphi^{*}, v$ and $w$ serve as a weak solution to problem (1.20). This completes the proof of Theorem 1.1.

\section{Approximate solutions. Time discretization}

In this section we construct sequences $\vartheta_{N}, v_{N}, \mathbf{u}_{N}, w_{N}, N \geq 1$, of approximate solutions to problem (1.20) by using time discretization. For given bounded functions $w_{n-2}, w_{n-1}, v_{n-1}$, and a vector field $\mathbf{f}$, we denote by $\mathbf{S}_{n}(\vartheta, \mathbf{u})$ the integral functional

$$
\mathbf{S}_{n}(\vartheta, \mathbf{u})=\boldsymbol{\Psi}_{g}\left(\mathbf{u}, \vartheta, w_{n-1}\right)-\frac{\tau}{2} \Pi_{0}(\vartheta, \vartheta)+\int_{\Omega}\left(w_{n-2}^{3} v_{n-1} \vartheta-\mathbf{f} \cdot \mathbf{u}\right) d x,
$$

where the free energy functional $\boldsymbol{\Psi}_{g}$ is given by (1.14) and the temperature energy dissipation rate $\Pi_{0}$ is given by (1.33). We are looking for the approximate solution to problem (1.20) in the form

$$
\begin{gathered}
\vartheta_{N}(x, t)=\vartheta_{n}(x), \quad v_{N}(x, t)=v_{n}(x), \\
\mathbf{u}_{N}(x, t)=\mathbf{u}_{n}(x), \quad w_{N}(x, t)=w_{n}(x, t)
\end{gathered}
$$

for

$$
t \in((n-1) \tau, n \tau], \quad 1 \leq n \leq N, \quad \tau=T N^{-1} .
$$

Set

$$
w_{n}(x)=w_{N}(x, \tau n)
$$

The functions $\vartheta_{n}, v_{n}$, and $\mathbf{u}_{n}$ are defined by the following recurrence relations. We assume that $v_{0}, w_{0}$ are given by the initial data $(1.20 \mathrm{~g})$ and

$$
\theta_{0}=\Theta\left(v_{0}, 0, w_{0}\right), \quad w_{-1}=0 .
$$


If $\vartheta_{n-1}, v_{n-1}$, and $w_{n-1}=w_{N}(x, \tau(n-1))$ are already determined for some $n \geq 1$, we define $\vartheta_{n}$ and $\mathbf{u}_{n}$ as solutions to the variational problem

$$
S_{n}\left(\vartheta_{n}, \mathbf{u}_{n}\right)=\min _{\mathbf{u}-\mathbf{h} \in \mathcal{W}^{2,2}} \max _{\vartheta \in W_{0}^{1,2}} \mathbf{S}_{n}(\vartheta, \mathbf{u})
$$

Then we define $v_{n}$ by

$$
v_{n}=\vartheta_{n}-W\left(w_{n-1}^{-1} D \mathbf{u}_{n}\right) \equiv V\left(D \mathbf{u}_{n}, \vartheta_{n}, w_{n-1}\right) .
$$

Next, we define $w_{N}$ on the interval $[\tau(n-1), \tau n]$ as a solution to the Cauchy problem

$$
\begin{aligned}
& \partial_{t} w_{N}=-H\left(\varphi\left(D^{2} \mathbf{u}_{n}, D \mathbf{u}_{n}, \vartheta_{n}, w_{N}\right)\right) w_{N}, \tau(n-1)<t \leq \tau n \\
& w_{N}(\tau(n-1))=w_{n-1}
\end{aligned}
$$

Then we define $w_{n}$ by $(2.3)$ and repeat the process until $n=N=T / \tau$. Finally, we define the approximation $\varphi_{N}$ of the trace of the Eshelby tensor by

$$
\varphi_{N}=\varphi\left(D^{2} \mathbf{u}_{N}, D \mathbf{u}_{N}, \vartheta_{N}, w_{N}\right)
$$

where $\varphi$ is given by $(1.20 \mathrm{e})$. Notice that $\mathbf{u}_{N}, \vartheta_{N}$ and $v_{N}$ are piecewise constant functions of the time variable. In contrast, $w_{N}$ is a Lipschitz continuous function of $t$. Relations (2.4)-(2.7) form a closed system of recurrent equations for the definition of approximate solution. The next theorem asserts the existence of solution to this system. In order to formulate this result, it is convenient to introduce the auxiliary functions

$$
\bar{w}_{N}(t)=w_{n-1}, \quad \bar{\vartheta}_{N}=\bar{\vartheta}_{n} \text { for }(n-1) \tau \leq t<n \tau
$$

where $\bar{\vartheta}_{n}$ is a solution to the variational problem

$$
\mathbf{S}_{n+1}\left(\mathbf{u}_{n}, \bar{\vartheta}_{n}\right)=\max _{\vartheta \in W^{1,2}(\Omega)} \mathbf{S}_{n+1}\left(\mathbf{u}_{n}, \vartheta\right)
$$

Theorem 2.1 Let conditions (H.1)-(H.3) be satisfied and $T>0$. Then there are $\tau_{0}>0$ and a positive constant $c$ with the following properties. For every integer $N>T / \tau_{0}$, problem (2.4)-(2.6) has a solution satisfying

$$
\begin{gathered}
\sup _{t} \int_{\Omega}\left(\left|\Delta \mathbf{u}_{N}\right|^{2}+W\left(w_{N}^{-1} D \mathbf{u}_{N}\right)+\left|\vartheta_{N}\right|^{2}\right) d x \\
+\int_{0}^{T} \Pi\left(\vartheta_{N}, \varphi_{N}\right) d t \leq c, \\
0<c^{-1} \leq w_{N}(x, t) \leq c, \quad\left|\partial_{t} w_{N}(x, t)\right| \leq c \text { a.e. in } \Omega \times[0, T], \\
\left|w_{N}-\bar{w}_{N}\right| \leq c \tau, \quad \int_{0}^{T-\tau} \int_{\Omega}\left|\vartheta_{N}-\bar{\vartheta}_{N}\right|^{2} \leq c \tau .
\end{gathered}
$$


Moreover, for every $0 \leq t_{1}<t_{0}<T$, we have

$$
\begin{aligned}
\limsup _{N \rightarrow \infty} & \left\{\mathcal{H}_{N}\left(t_{1}\right)-\mathcal{H}_{N}\left(t_{0}\right)+\int_{t_{1}+\tau}^{t_{0}} \Pi_{1}\left(H\left(\varphi_{N}\right), \varphi_{N}\right)\right. \\
& \left.+\frac{1}{2} \int_{t_{1}+\tau}^{t_{0}} \Pi_{0}\left(\vartheta_{N}, \vartheta_{N}\right) d s+\frac{1}{2} \int_{t_{1}+\tau}^{t_{0}-\tau} \Pi_{0}\left(\bar{\vartheta}_{N}, \bar{\vartheta}_{N}\right) d s\right\} \leq 0 .
\end{aligned}
$$

Here

$$
\mathcal{H}_{N}(t)=\mathcal{E}\left(\mathbf{u}_{N}(t), v_{N}(t), \bar{w}_{N}(t)\right)-\int_{\Omega} \mathbf{f} \cdot \mathbf{u}_{N}(t) d x,
$$

the total internal energy $\mathcal{E}$ and the forms $\Pi_{i}$ are given by (1.33) and (1.32), and the trace of the Eshelby tensor $\varphi_{N}$ is given by (2.8).

Proof The proof is in Appendix A.

Theorem 2.1 implies that the functions $\left(w_{N}\right)^{ \pm 1}$ are uniformly bounded and the functions $\mathbf{u}_{N}, \vartheta_{N}$ satisfy the estimates

$$
\left\|\mathbf{u}_{N}\right\|_{L^{\infty}\left(0, T ; \mathcal{W}^{2,2}\right)}+\left\|\vartheta_{N}\right\|_{L^{2}\left(0, T ; W^{1,2}(\Omega)\right)}+\left\|\vartheta_{N}\right\|_{L^{\infty}\left(0, T ; L^{2}(\Omega)\right)} \leq c,
$$

where $c$ is independent of $N$. Now we use bootstrap arguments to obtain stronger estimates. In particular we estimate $\mathbf{u}_{N}$ in $L^{\infty}\left(0, T ; W^{2,6}(\Omega)\right)$ and estimate the derivatives of $w_{N}$. The corresponding result is

Theorem 2.2 Under the assumptions of Theorem 2.1,

$$
\begin{aligned}
& \sup _{t \in[0, T]}\left(\left\|\mathbf{u}_{N}(t)\right\|_{W^{2,6}(\Omega)}+\left\|D \mathbf{u}_{N}(t)\right\|_{L^{\infty}(\Omega)}\right) \leq c, \\
& \sup _{t \in[0, T]}\left\|\bar{w}_{N}(t)^{-1} \Delta \mathbf{u}_{N}(t)\right\|_{W^{1,2}(\Omega)} \leq c, \\
& \sup _{t \in[0, T]}\left\|w_{N}(t)\right\|_{W^{1,2}(\Omega)}+\left\|\partial_{t} w_{N}\right\|_{L^{2}\left(0, T ; W^{1,2}(\Omega)\right)} \leq c,
\end{aligned}
$$

where the constant $c$ is independent of $N$.

The following proposition gives estimates of the approximate solutions in Lebesgue spaces. It is a straightforward consequence of Theorem 2.2. Introduce the exponent $(r, p)$ and $(s, q)$ satisfying the relations

$$
\begin{aligned}
& 1<s<\infty, \quad 1<q<6 s /(6 s-4) \\
& r=2 s, \quad p=2 q
\end{aligned}
$$

It follows that

$$
2<r<\infty, \quad 1<p<6 r /(3 r-4) .
$$

Proposition 2.1 Under the assumptions of Theorem 2.1,

$$
\begin{aligned}
& \left\|v_{N}\right\|_{L^{\infty}\left(0, T ; L^{2}(\Omega)\right.}+\left\|v_{N}\right\|_{L^{2}\left(0, T ; W^{1,2}(\Omega)\right.} \leq c \\
& \left\|\vartheta_{N}\right\|_{L^{r}\left(0, T ; L^{p}(\Omega)\right.}+\left\|v_{N}\right\|_{L^{r}\left(0, T ; L^{p}(\Omega)\right.} \leq c \\
& \left\|\varphi_{N}\right\|_{L^{s}\left(0, T ; L^{q}(\Omega)\right)} \leq c
\end{aligned}
$$

where $c$ is independent of $N$.

The rest of this section is devoted to the proof of Theorem 2.2 and Proposition 2.1 . 
Proof of Theorem 2.2. We begin with the proof of estimate (2.17). The proof is based on the fact that the growth exponent $\varkappa \in[2,3)$ in $(1.21)$ is less than 3 . Since the case $\varkappa=2$ is trivial, we assume $\varkappa \in(2,3)$. By the definition of the approximate solution, we have

$$
\mathbf{S}_{n}\left(\mathbf{u}_{n}, \vartheta_{n}\right)=\min _{\mathbf{u}-\mathbf{h} \in \mathcal{W}^{2,2}} \mathbf{S}\left(\mathbf{u}, \vartheta_{n}\right)
$$

It follows that

$$
\lim _{\delta \rightarrow 0} \delta^{-1}\left(\mathbf{S}_{n}\left(\mathbf{u}_{n}-\delta \mathbf{q}, \vartheta_{n}\right)-\mathbf{S}_{n}\left(\mathbf{u}_{n}, \vartheta_{n}\right)\right)=0
$$

for every $\mathbf{q} \in \mathcal{W}^{2,2}$. This relation can be rewritten in the form

$$
\int_{\Omega}\left(\frac{\varepsilon}{2 w_{n-1}} \Delta \mathbf{u}_{n} \Delta \mathbf{q}+w_{n-1}^{2}\left(1+\vartheta_{n}\right) W\left(w_{n-1}^{-1} D \mathbf{u}_{n}\right): D \mathbf{q}-f \mathbf{q}\right) d x=0 .
$$

Now choose $\boldsymbol{\xi} \in L^{2}(\Omega)$ and set $\mathbf{q}=\Delta^{-1} \boldsymbol{\xi}$, where the inverse $\Delta^{-1}$ is defined as the solution to the Dirichlet problem

$$
\Delta\left(\Delta^{-1} \boldsymbol{\xi}\right)=\boldsymbol{\xi} \text { in } \Omega, \quad \Delta^{-1} \boldsymbol{\xi}=0 \text { on } \partial \Omega .
$$

Thus we get

$$
\int_{\Omega}\left(\frac{\varepsilon}{2 w_{n-1}} \Delta \mathbf{u}_{n}-\Delta^{-1} \operatorname{div}\left(w_{n-1}^{2}\left(1+\vartheta_{n}\right) W\left(w_{n-1}^{-1} D \mathbf{u}_{n}\right)\right)-f\right) \boldsymbol{\xi} d x=0,
$$

which yields the equation

$$
\frac{\varepsilon}{2 w_{n-1}} \Delta \mathbf{u}_{n}=\Delta^{-1} \operatorname{div}\left\{w_{n-1}^{2}\left(1+\vartheta_{n}\right) W\left(w_{n-1}^{-1} D \mathbf{u}_{n}\right)\right\}+\Delta^{-1} f .
$$

Since $\mathbf{u}_{n}-\mathbf{h} \in \mathcal{W}^{2,2}$, we also have

$$
\mathbf{u}_{n}=\mathbf{h} \text { on } \partial \Omega .
$$

It follows from the general theory of elliptic equations [15] that for all $\mathbf{q} \in$ $W^{k-1, p}(\Omega), k \geq 1, p \in(1, \infty)$, we have

$$
\left\|\Delta^{-1} \operatorname{div} \mathbf{q}\right\|_{W^{k, p}(\Omega)} \leq c\|\mathbf{q}\|_{W^{k-1, p}(\Omega)},
$$

where $c$ is independent of $\mathbf{q}$. Since the functions $w_{N}^{ \pm 1}$ are uniformly bounded, it follows from the growth condition (1.21) that

$$
\left|w_{n-1}^{2}\left(1+\vartheta_{n}\right) W\left(w_{n-1}^{-1} D \mathbf{u}_{n}\right)\right| \leq c\left(1+\left|\vartheta_{n}\right|\right)\left(1+\left|D \mathbf{u}_{n}\right|\right)^{\gamma},
$$

where $\gamma=\varkappa-1 \in(1,2)$. Now set $\beta_{0}=2, \alpha_{0}=6$. The energy estimate $(2.16)$ implies

$$
\left\|\Delta \mathbf{u}_{n}\right\|_{L^{\beta_{0}(\Omega)}} \leq c, \quad\left\|D \mathbf{u}_{n}\right\|_{L^{\alpha_{0}(\Omega)}} \leq c .
$$

It follows from this, estimate (2.16), and the Hölder inequality that

$$
\begin{aligned}
\|\left(1+\left|\vartheta_{n}\right|\right)\left(1+\left|D \mathbf{u}_{n}\right|\right)^{\gamma} & \|_{L^{p_{0}(\Omega)}} \\
& \leq c\left(1+\left\|\vartheta_{n}\right\|_{L^{2}(\Omega)}\right)\left(1+\left\|D \mathbf{u}_{n}\right\|_{L^{6}(\Omega)}\right)^{\gamma} \leq c,
\end{aligned}
$$


where $p_{0}^{-1}=2^{-1}+\gamma 6^{-1}<5 / 6$. Combining this estimate with $(2.26)$ we arrive at the inequality

$$
\left\|\Delta^{-1} \operatorname{div}\left\{w_{n-1}^{2}\left(1+\vartheta_{n}\right) W\left(w_{n-1}^{-1} D \mathbf{u}_{n}\right)\right\}\right\|_{W^{1, p_{0}(\Omega)}} \leq c .
$$

Since the embedding $W^{1, p_{0}}(\Omega) \hookrightarrow L^{3 p_{0} /\left(3-p_{0}\right)}(\Omega)$ is bounded we conclude from this and (2.24)-(2.25) that

$$
\left\|\Delta \mathbf{u}_{n}\right\|_{L^{\beta_{1}(\Omega)}} \leq c, \text { where } \beta_{1}=3 p_{0} /\left(3-p_{0}\right) .
$$

Since the embedding $\mathcal{W}^{2, \beta_{1}} \hookrightarrow W^{1,3 \beta_{1} /\left(3-\beta_{1}\right)}(\Omega)$ is bounded, we have

$$
\left\|D \mathbf{u}_{n}\right\|_{L^{\alpha_{1}(\Omega)}} \leq c, \text { where } \alpha_{1}=3 \beta_{1} /\left(3-\beta_{1}\right) .
$$

Applying the Hölder inequality we arrive at

$$
\begin{aligned}
\left\|\left(1+\left|\vartheta_{n}\right|\right)\left(1+\left|D \mathbf{u}_{n}\right|\right)^{\gamma}\right\|_{L^{p_{1}(\Omega)}} & \\
& \leq c\left(1+\left\|\vartheta_{n}\right\|_{L^{2}(\Omega)}\right)\left(1+\left\|D \mathbf{u}_{n}\right\|_{L^{\alpha_{1}}(\Omega)}\right)^{\gamma} \leq c,
\end{aligned}
$$

where $p_{1}^{-1}=2^{-1}+\gamma \alpha_{1}^{-1}$. Arguing as before we conclude that

$$
\left\|\Delta \mathbf{u}_{n}\right\|_{L^{\beta_{k}(\Omega)}} \leq c, \quad\left\|D \mathbf{u}_{n}\right\|_{L^{\alpha}(\Omega)} \leq c .
$$

Here the sequences $\alpha_{k}, p_{k}$, and $\beta_{k}$ are defined by the recurrent relations

$$
\alpha_{k}=3 \beta_{k-1}\left(3-\beta_{k-1}\right)^{-1}, \beta_{k}=3 p_{k-1}\left(3-p_{k-1}\right)^{-1}, \quad p_{k}^{-1}=2^{-1}+\gamma \alpha_{k-1}^{-1} .
$$

Estimates (2.29) hold provided $1 \leq \beta_{k-1}<3$ or equivalently $1 \leq p_{k}<3 / 2$. Notice that the quantities $p_{k}$ are defined by the recurrent relations $p_{k}^{-1}=$ $\gamma p_{k-1}^{-1}+1 / 2-2 \gamma / 3$, which lead to the equality

$$
p_{k}^{-1}=\gamma^{k} p_{0}^{-1}-(4 \gamma-3) \frac{\gamma^{k}-1}{6(\gamma-1)}=\frac{1}{6(\gamma-1)}\left(\gamma^{k+1}(\gamma-2)+(4 \gamma-3)\right) \text {. }
$$

Since $\gamma \in(1,2)$, the sequence $p_{k}^{-1}$ decreases and tends to $-\infty$ as $k \rightarrow \infty$. Hence there is a minimal $k$ such that $p_{k-1} \leq 3 / 2$ and $p_{k}>3 / 2$. It follows that $\beta_{k+1}>3$. In this case the embedding $\mathcal{W}^{2, \beta_{k+1}} \hookrightarrow L^{\infty}(\Omega)$ is bounded, which yields

$$
\left\|D \mathbf{u}_{n}\right\|_{L^{\infty}(\Omega)} \leq c, \quad\left\|\left(1+\left|\vartheta_{n}\right|\right)\left(1+\left|D \mathbf{u}_{n}\right|\right)^{\gamma}\right\|_{L^{2}(\Omega)} \leq c .
$$

We thus get

$$
\left\|\Delta^{-1} \operatorname{div}\left\{w_{n-1}^{2}\left(1+\vartheta_{n}\right) W\left(w_{n-1}^{-1} D \mathbf{u}_{n}\right)\right\}\right\|_{W^{1,2}(\Omega)} \leq c .
$$

From this and (2.24) we conclude that

$$
\left\|w_{n-1}^{-1} \Delta \mathbf{u}_{n}\right\|_{W^{1,2}(\Omega)} \leq c
$$

and hence

$$
\left\|\mathbf{u}_{n}\right\|_{W^{2,6}(\Omega)} \leq c
$$


It remains to note that estimates (2.17) and (2.18) obviously follow from (2.30)-(2.32).

Now our task is to estimate $w_{N}$. Recall that $w_{N}$ satisfies the ordinary differential equation and initial condition (2.7). Notice that the differentiability of $w_{N}$ with respect to $x$ follows from the differentiability of $\mathbf{u}_{n}, \vartheta_{n}$, and general results on the differentiability of solutions to ordinary differential equations with respect to parameters. It is necessary to prove (2.17). Differentiation of both sides of (2.7) with respect to $x$ gives

$$
\begin{aligned}
& \partial_{t}\left(\nabla w_{N}\right)=-H(\varphi) \nabla w_{N}-H^{\prime}(\varphi) w_{N} \nabla \varphi \text { for } \tau(n-1)<t \leq \tau n, \\
& \nabla w_{N}(\tau(n-1))=\nabla w_{n-1} .
\end{aligned}
$$

Next, formula (1.20e) for $\varphi$ implies

$$
\begin{aligned}
& \varphi\left(D^{2} \mathbf{u}_{n}, D \mathbf{u}_{n}, \vartheta_{n}, w_{N}\right)=-\varepsilon w_{n-1}^{2}\left(2 w_{N}\right)^{-1} \sigma_{n}^{2} \\
& +\left(1+\vartheta_{n}\right) w_{N}^{3}\left(3 W\left(w_{N}^{-1} D \mathbf{u}_{n}\right)-W^{\prime}\left(w_{N}^{-1} D \mathbf{u}_{n}\right):\left(w_{N}^{-1} D \mathbf{u}_{n}\right)\right)-\frac{3 w_{N}^{3}}{2} \vartheta_{n}^{2},
\end{aligned}
$$

where

$$
\sigma_{n}=w_{n-1}^{-1} \Delta \mathbf{u}_{n}=\left(\bar{w}_{N}(t)\right)^{-1} \Delta \mathbf{u}_{N}(t), \quad t \in((n-1) \tau, n \tau] .
$$

It follows that

$$
\nabla \varphi=\mathbf{M} \nabla w_{N}+\mathbf{N} \nabla w_{n-1}+\mathbf{L}
$$

where

$$
\begin{aligned}
\mathbf{M}= & w_{N}^{2}\left(1+\vartheta_{n}\right) \mathbf{R}(\boldsymbol{\xi})+\frac{\varepsilon}{2 w_{N}^{2}}\left(\Delta \mathbf{u}_{n}\right)^{2}-\frac{9}{2} w_{N}^{2} \vartheta_{n}^{2}, \quad \mathbf{N}=-\frac{\varepsilon w_{n-1}}{w_{N}} \sigma_{n} \\
\mathbf{L}= & w_{N}^{2}\left(1+\vartheta_{n}\right) \mathbf{P}(\boldsymbol{\xi}, \boldsymbol{\eta})+w_{N}^{3}\left(3 W(\boldsymbol{\xi})-W^{\prime}(\boldsymbol{\xi}): \boldsymbol{\xi}\right) \nabla \vartheta_{n} \\
& -\frac{\varepsilon w_{n-1}^{2}}{w_{N}} \sigma_{n} \nabla \sigma_{n}-3 w_{N}^{3} \vartheta_{n} \nabla \vartheta_{n} .
\end{aligned}
$$

Here the matrix valued function $\boldsymbol{\xi}$ and the vector-matrix valued function $\boldsymbol{\eta}$ are given by

$$
\boldsymbol{\xi}=w_{N}^{-1} D \mathbf{u}_{n}, \quad \boldsymbol{\eta}=\left(\boldsymbol{\eta}_{i}\right)_{1 \leq i \leq 3}, \quad \boldsymbol{\eta}_{i}=w_{N}^{-1} \partial_{x_{i}} D \mathbf{u}_{n},
$$

and the scalar function $\mathbf{R}$ and the vector function $\mathbf{P}=\left(P_{i}\right)_{1 \leq i \leq 3}$ are given by

$$
\begin{aligned}
\mathbf{R} & =9 W(\boldsymbol{\xi})-5 W^{\prime}(\boldsymbol{\xi}): \boldsymbol{\xi}+W^{\prime \prime}(\boldsymbol{\xi})[\boldsymbol{\xi}, \boldsymbol{\xi}], \\
P_{i} & =2 W^{\prime}(\xi): \boldsymbol{\eta}_{i}-W^{\prime \prime}(\boldsymbol{\xi})\left[\boldsymbol{\xi}, \boldsymbol{\eta}_{i}\right] .
\end{aligned}
$$

Since the functions $w_{N}$ and $D \mathbf{u}_{n}$ are uniformly bounded we have

$$
|\boldsymbol{\xi}| \leq c, \quad|\boldsymbol{\eta}| \leq c\left|\nabla^{2} \mathbf{u}_{n}\right|, \quad|\mathbf{R}| \leq c, \quad|\mathbf{P}| \leq c\left|\nabla^{2} \mathbf{u}_{n}\right| .
$$


It follows that

$$
\begin{aligned}
|\mathbf{M}| & \leq c\left(1+\left|\vartheta_{n}\right|^{2}+\left|\Delta \mathbf{u}_{n}\right|^{2}\right), \quad|\mathbf{N}| \leq c\left|\Delta \mathbf{u}_{n}\right|, \\
|\mathbf{L}| & \leq c\left(1+\left|\vartheta_{n}\right|\right)\left(1+\left|\nabla^{2} \mathbf{u}_{n}\right|\right)+c\left|\Delta \mathbf{u}_{n}\right|\left|\nabla \sigma_{n}\right|+c\left(1+\left|\vartheta_{n}\right|\right)\left|\nabla \vartheta_{n}\right| .
\end{aligned}
$$

On the other hand, representation (2.34) implies

$$
-\varphi\left(D^{2} \mathbf{u}_{n}, D \mathbf{u}_{n}, \vartheta_{n}, w_{N}\right) \geq c^{-1}\left(\left|\vartheta_{n}\right|^{2}+\left|\Delta \mathbf{u}_{n}\right|^{2}\right)-c,
$$

where $c>0$ is independent of $N$. From this and $\left|H^{\prime}(\varphi)\right| \leq c(1+|\varphi|)^{-1}$ we conclude that

$$
\left|H^{\prime}(\varphi)\right| \leq c\left(1+\left|\vartheta_{n}\right|^{2}+\left|\Delta \mathbf{u}_{n}\right|^{2}\right)^{-1} .
$$

Combining this with $(2.41)$ we arrive at

$$
\begin{aligned}
\left|H^{\prime}(\varphi) \mathbf{M}\right| & \leq c, \quad\left|H^{\prime}(\varphi) \mathbf{N}\right| \leq c, \\
\left|H^{\prime}(\varphi) \mathbf{L}\right| & \leq c\left(1+\left|\nabla \sigma_{n}\right|+\left|\nabla \vartheta_{n}\right|+\left|\nabla^{2} \mathbf{u}_{n}\right|\right) .
\end{aligned}
$$

Next, substituting (2.37) into (2.33) we obtain

$\left.\partial_{t}\left(\nabla w_{N}\right)=-\left(H(\varphi)+H^{\prime}(\varphi) w_{N} \mathbf{M}\right) \nabla w_{N}-w_{N} H^{\prime}(\varphi) \mathbf{N}\right) \nabla w_{n-1}-w_{N} H^{\prime}(\varphi) \mathbf{L}$, which along with (2.41) yields

$$
\partial_{t}\left|\nabla w_{N}\right| \leq\left|\partial_{t}\left(\nabla w_{N}\right)\right| \leq c\left|\nabla w_{N}\right|+c\left|\nabla w_{n-1}\right|+c G_{n} .
$$

Here

$$
G_{n}=1+\left|\nabla^{2} \mathbf{u}_{n}\right|+\left|\nabla \vartheta_{n}\right|+\left|\nabla \sigma_{n}\right|
$$

Multiplying both sides of $(2.42)$ by $\exp (-c t)$ we obtain

$$
\partial_{t}\left(e^{-c t}\left|\nabla w_{N}\right|\right) \leq c e^{-c t}\left(\left|\nabla w_{n-1}\right|+c\left(1+\left|\nabla^{2} \mathbf{u}_{n}\right|+\left|\nabla \vartheta_{n}\right|+\left|\nabla \sigma_{n}\right|\right) .\right.
$$

Choose any $t \in[\tau(n-1), \tau n]$. Integrating this inequality over $[\tau(n-1), t]$ and multiplying the result by $e^{c t}$, we arrive at

$$
\begin{aligned}
\left|\nabla w_{N}(t)\right| & \leq\left|\nabla w_{n-1}\right|+\left(e^{t-(n-1) \tau}-1\right)\left(2\left|\nabla w_{n-1}\right|+G_{n}\right) \\
& \leq\left|\nabla w_{n-1}\right|(1+c \tau)+c \tau G_{n},
\end{aligned}
$$

where $c$ is independent of $N$. Applying the Cauchy inequality we obtain

$$
\left|\nabla w_{N}(t)\right|^{2} \leq\left|\nabla w_{n-1}\right|^{2}(1+c \tau)+c \tau G_{n}^{2} \quad \text { for } \tau(n-1) \leq t \leq \tau n .
$$

Integrating both sides over $\Omega$ and recalling estimates (2.17)-(2.18) we obtain

$$
\begin{array}{rl}
\int_{\Omega}\left|\nabla w_{N}(t)\right|^{2} & d x \leq(1+c \tau) \int_{\Omega}\left|\nabla w_{n-1}\right|^{2} d x+c \tau \int_{\Omega} G_{n}^{2} d x \\
\leq & (1+c \tau) \int_{\Omega}\left|\nabla w_{n-1}\right|^{2} d x+c \tau \int_{\Omega}\left(1+\left|\nabla \vartheta_{n}\right|^{2}\right) d x
\end{array}
$$

Since $w_{N}(n \tau)=w_{n}$, we conclude from this that

$$
\int_{\Omega}\left|\nabla w_{n}\right|^{2} d x \leq(1+c \tau) \int_{\Omega}\left|\nabla w_{n-1}\right|^{2} d x+c \tau \int_{\Omega}\left(1+\left|\nabla \vartheta_{n}\right|^{2}\right) d x
$$


It follows that

$\int_{\Omega}\left|\nabla w_{n}\right|^{2} d x \leq(1+c \tau)^{n} \int_{\Omega}\left|\nabla w_{0}\right|^{2} d x+c \tau \sum_{k=0}^{n}(1+c \tau)^{n-k} \int_{\Omega}\left(1+\left|\nabla \vartheta_{k}\right|^{2} d x\right.$.

In view of the relation $\tau=T N^{-1}$ we have

$$
(1+c \tau)^{n} \leq(1+c \tau)^{N}=\left\{(1+c \tau)^{1 /(c \tau)}\right\}^{c T} \leq e^{c T} .
$$

Thus we get

$$
\begin{array}{r}
\int_{\Omega}\left|\nabla w_{n}\right|^{2} d x \leq e^{c T} \int_{\Omega}\left|\nabla w_{0}\right|^{2} d x+c e^{c T} \tau \sum_{k=0}^{n} \int_{\Omega}\left(1+\left|\nabla \vartheta_{k}\right|^{2}\right) d x \\
=e^{c T} \int_{\Omega}\left|\nabla w_{0}\right|^{2} d x+c e^{c T} \int_{0}^{n \tau} \int_{\Omega}\left(1+\left|\nabla \vartheta_{k}\right|^{2}\right) d x d t \leq c
\end{array}
$$

for all $1 \leq n \leq N$. Combining this result with (2.45) we obtain

$$
\int_{\Omega}\left|\nabla w_{N}(t)\right|^{2} d x \leq c \text { for all } t \in[0, T] .
$$

From this, (2.46), and (2.42) we conclude that

$$
\int_{\Omega}\left|\partial_{t}\left(\nabla w_{N}(t)\right)\right|^{2} d x \leq c+c \int_{\Omega}\left|\nabla \vartheta_{N}\right|^{2} d x
$$

for $\tau(n-1)<t \leq \tau n$. Noting that $\vartheta_{N}(t)=\vartheta_{n}$ on this interval, we obtain

$$
\int_{0}^{T} \int_{\Omega}\left|\partial_{t}\left(\nabla w_{N}(t)\right)\right|^{2} d x d t \leq c+c \int_{0}^{T} \int_{\Omega}\left|\nabla \vartheta_{N}\right|^{2} d x \leq c .
$$

It remains to note that the desired inequality (2.19) clearly follows from (2.47) and (2.48).

Proof of Proposition 2.1. We first observe that estimate (2.21) obviously follows from (2.16)-(2.19) and the identity $v_{N}=\vartheta_{N}-W\left(w_{N}^{-1} D \mathbf{u}_{N}\right)$.

Let us prove estimate (2.22). Since the embedding $W^{1,2}(\Omega) \hookrightarrow L^{6}(\Omega)$ is bounded, the energy estimate (2.16) yields

$$
\left\|\vartheta_{N}\right\|_{L^{\infty}\left(0, T ; L^{2}(\Omega)\right)}+\left\|\vartheta_{N}\right\|_{L^{2}\left(0, T ; L^{6}(\Omega)\right)} \leq c .
$$

By the interpolation inequality, for every $\alpha \in(0,1)$ we have

$$
\left\|\vartheta_{N}\right\|_{L^{r}\left(0, T ; L^{p}(\Omega)\right.} \leq\left\|\vartheta_{N}\right\|_{L^{\infty}\left(0, T ; L^{2}(\Omega)\right.}^{1-\alpha}\left\|\vartheta_{N}\right\|_{L^{2}\left(0, T ; L^{6}(\Omega)\right.}^{\alpha} \leq c,
$$

where

$$
\alpha / 2=1 / r, \quad(1-\alpha) / 2+\alpha / 6=1 / p .
$$

Estimate (2.22) for $\vartheta_{N}$ obviously follows from (2.49). Repeating these arguments and using (2.21) we obtain (2.22) for $v_{N}$. 
It remains to prove estimate (2.23). Recall representation (2.8) for $\varphi_{N}$. Since $w_{n}^{ \pm 1}$ and $D \mathbf{u}_{n}$ are uniformly bounded, it follows from (2.8) that for almost every $t \in(0, T)$,

$$
\left|\varphi_{N}(t)\right| \leq c+c\left|\Delta \mathbf{u}_{N}(t)\right|^{2}+c\left|\vartheta_{N}(t)\right|^{2}
$$

Notice that in view of $(2.16)$ we have

$$
\left\|\left(\Delta \mathbf{u}_{N}\right)^{2}\right\|_{L^{s}\left(0, T ; L^{q}(\Omega)\right)} \leq c\left\|\Delta \mathbf{u}_{N}\right\|_{L^{\infty}\left(0, T ; L^{6}(\Omega)\right)} \leq c .
$$

Next, relation (2.20) yields $2 s=r$ and $2 q=p$. From this and estimate (2.49) we obtain

$$
\left\|\vartheta_{N}^{2}\right\|_{L^{s}\left(0, T ; L^{q}(\Omega)\right)} \leq c\left\|\vartheta_{N}\right\|_{L^{r}\left(0, T ; L^{p}(\Omega)\right)}^{2} \leq c .
$$

Combining (2.50)-(2.52) we arrive at estimate (2.23).

\section{Compactness}

In Section 2 we proved the existence of approximate solutions $\mathbf{u}_{N}, \vartheta_{N}, v_{N}$, $w_{N}$ to problem (1.20). Our goal is to prove that this sequence has a limit point which is a weak solution to (1.20). Hence, the key question is the compactness of the set of approximate solutions in appropriate Banach spaces. In this section we give a preliminary analysis of this problem. Notice that among the thermodynamical and mechanical quantities in (1.20), only the entropy $v$ and the growth factor $w$ satisfy evolution equations. Therefore, the compactness properties of the sequences $v_{N}$ and $w_{N}$ can be established by applying the Dubinski-Lions Lemma. The corresponding result is given by the following theorem, which is the first main result of this section.

Theorem 3.1 Let all conditions of Theorem 2.1 be satisfied. Then there is a subsequence of $\left(v_{N}, w_{N}\right)$, still denoted by $\left(v_{N}, w_{N}\right)$, and functions $v, w$ with

$$
\begin{gathered}
w^{ \pm 1} \in L^{\infty}\left(0, T ; L^{\infty}(\Omega)\right) \cap L^{\infty}\left(0, T ; W^{1,2}(\Omega)\right), \partial_{t} w \in L^{2}\left(0, T ; W^{1,2}(\Omega)\right), \\
v \in L^{2}\left(0, T ; W^{1,2}(\Omega)\right) \cap L^{\infty}\left(0, T ; L^{2}(\Omega)\right)
\end{gathered}
$$

such that

$$
\begin{gathered}
w_{N}^{ \pm 1} \rightarrow w^{ \pm 1} \quad \text { in } C\left(0, T ; L^{\alpha}(\Omega)\right) \\
v_{N} \rightarrow v \quad \text { in } L^{r}\left(0, T ; L^{p}(\Omega)\right)
\end{gathered}
$$

as $N \rightarrow \infty$ for all $\alpha \in[1, \infty)$ and all $(p, r)$ satisfying $(2.20)$.

We cannot guarantee the strong convergence of the sequences $\mathbf{u}_{N}, \vartheta_{N}$ and $\varphi_{N}$ since these functions have no smoothness with respect to the time variable. However, they have some smoothness with respect to the spatial variables. Hence we can expect that these functions map the interval $(0, T)$ onto some relatively compact set. The corresponding result is given by the following theorem, which is the second main result of this section.

Theorem 3.2 Let exponents $s, q$ and $r, p$ satisfy conditions (2.20). Then for every $\eta>0$ there is a compact set $\mathcal{T}_{\eta}$ with the following properties: 
(i) $\mathcal{T}_{\eta} \subset(0, T)$, meas $\left((0, T) \backslash \mathcal{T}_{\eta}\right) \leq \eta$.

(ii) The mappings $v: \mathcal{T}_{\tau} \rightarrow L^{p}(\Omega)$ and $v_{N}: \mathcal{T}_{\tau} \rightarrow L^{p}(\Omega)$ are continuous and

$$
v_{N}(t) \rightarrow v(t) \quad \text { in } L^{p}(\Omega) \text { uniformly on } \mathcal{T}_{\eta} .
$$

(iii) For every $\alpha \in[1, \infty)$,

$$
w_{N}(t) \rightarrow w(t) \text { in } L^{\alpha}(\Omega) \text { uniformly on } \mathcal{T}_{\eta} .
$$

(iv) The set

$$
\mathfrak{T}(\eta)=\left\{\left(\vartheta_{N}(t), \varphi_{N}(t)\right): N \geq 1, t \in \mathcal{T}_{\eta}\right\}
$$

is relatively compact in $L^{p}(\Omega) \times L^{q}(\Omega)$.

The rest of this section is devoted to the proof of Theorems 3.1 and 3.2.

Proof of Theorem 3.1. We begin by proving (3.2). It suffices to show that the sequence $\left\{w_{N}(t)\right\}_{N \geq 1}$ is relatively compact in $C\left(0, T ; L^{\alpha}(\Omega)\right)$ for every $\alpha \in[1, \infty)$. Set

$$
\mathfrak{M}_{w}=\left\{w_{N}(t): t \in[0, T], N \geq 1\right\} .
$$

It follows from (2.17) that $\mathfrak{M}_{w}$ is bounded in $W^{1,2}(\Omega)$ and hence in $L^{r}(\Omega)$ for every $r \in[1,6)$. In particular, it is relatively compact in measure. On the other hand, inequality (2.17) yields the boundedness of $\mathfrak{M}_{w}$ in $L^{\infty}(\Omega)$. Hence $\mathfrak{M}_{w}$ is relatively compact in $L^{\alpha}(\Omega)$ for all $\alpha \in[1, \infty)$.

Next, it follows from estimate (2.19) that for $1 \leq \alpha \leq 6, h \in(0, T)$, and $0 \leq t \leq T-h$,

$$
\begin{aligned}
\left\|w_{N}(t+h)-w_{N}(t)\right\|_{L^{\alpha}(\Omega)} & \leq c\left\|w_{N}(t+h)-w_{N}(t)\right\|_{W^{1,2}(\Omega)} \\
\leq c\left\|\int_{t}^{t+h} \partial_{s} w_{N}(s) d s\right\| & \leq h^{1 / 2}\left\|\partial_{t} w_{N}(t)\right\|_{L^{2}\left(0, T ; W^{1,2}(\Omega)\right)} \leq c h^{1 / 2} .
\end{aligned}
$$

If $\alpha>6$ we apply the interpolation inequality to obtain

$$
\begin{aligned}
\| w_{N}(t+ & h)-w_{N}(t) \|_{L^{\alpha}(\Omega)} \\
& \leq c\left\|w_{N}(t+h)-w_{N}(t)\right\|_{L^{6}(\Omega)}^{6 / \alpha}\left\|w_{N}(t+h)-w_{N}(t)\right\|_{L^{\infty}(\Omega)}^{(\alpha-6) / \alpha} \\
& \leq c\left\|w_{N}(t+h)-w_{N}(t)\right\|_{L^{6}(\Omega)}^{6 / \alpha} \leq c h^{3 / \alpha} .
\end{aligned}
$$

Estimates (3.7) and (3.8) show that the sequence $w_{N}$ is equicontinuous in $C\left(0, T ; L^{\alpha}(\Omega)\right)$. Recall that $w_{N}$ takes values in the relatively compact set $\mathfrak{M}_{w}$. Application of the Ascoli Theorem completes the proof of (3.2).

Our next task is to prove (3.3). Recall that $\left(\mathbf{u}_{n}, \vartheta_{n}\right)$ is a solution to the variational problem

$$
\mathbf{S}_{n}\left(\mathbf{u}_{n}, \vartheta_{n}\right)=\max _{\vartheta \in W^{1,2}(\Omega)} \mathbf{S}_{n}\left(\mathbf{u}_{n}, \vartheta\right) .
$$

Calculation of the variation of $\mathbf{S}_{n}$ at the point $\vartheta_{n}$ leads to the linear elliptic boundary value problem for $\vartheta_{n}$,

$$
\begin{aligned}
& -\tau \Delta \vartheta_{n}+w_{n-1}^{3} V\left(D \mathbf{u}_{n}, \vartheta_{n}, w_{n-1}\right)=w_{n-2}^{3} v_{n-1} \text { in } \Omega, \\
& \partial_{n} \vartheta_{n}+\vartheta_{n}=0 \text { on } \partial \Omega .
\end{aligned}
$$


Here $V$ is given by $(1.27)$, i.e.,

$$
V\left(D \mathbf{u}_{n}, \vartheta_{n}, w_{n-1}\right)=\vartheta_{n}-W\left(w_{n-1}^{-1} D \mathbf{u}_{n}\right)=v_{n} .
$$

It follows that

$$
v_{n}-v_{n-1}=w_{n-1}^{-3}\left(\tau \Delta \vartheta_{n}+R_{n}\right) \text {, where } R_{n}=\left(w_{n-2}^{3}-w_{n-1}^{3}\right) v_{n-1} .
$$

Recall that $w_{n-2}=w_{N}((n-2) \tau)$ and $w_{n-1}=w_{N}((n-1) \tau)$. From this and (2.19) we conclude that $\left|w_{n-2}^{3}-w_{n-1}^{3}\right| \leq c \tau$ and hence

$$
\left|R_{n}\right| \leq c \tau\left|v_{n-1}\right| \text {. }
$$

In view of (2.19), we have $\left|w_{n-1}^{ \pm 1}\right| \leq c$ and $\left\|\nabla w_{n-1}\right\|_{L^{2}(\Omega)} \leq c$. Fix $\lambda>3$. Since the embedding $W_{0}^{1, \lambda}(\Omega) \hookrightarrow L^{\infty}(\Omega)$ is bounded, we have

$$
\left\|w_{n-1}^{-3} \zeta\right\|_{W^{1,2}(\Omega)} \leq c\|\zeta\|_{W_{0}^{1, \lambda}(\Omega)} \quad \text { for all } \zeta \in W_{0}^{1, \lambda}(\Omega) .
$$

Obviously $\zeta w_{n-1}^{-3} \in W_{0}^{1,2}(\Omega)$. Thus we get

$$
\int_{\Omega} \zeta w_{n-1}^{-3} \Delta \vartheta_{n} d x=-\int_{\Omega} \nabla\left(\zeta w_{n-1}^{-3}\right) \nabla \vartheta_{n} d x \leq c\left\|\nabla \vartheta_{n}\right\|_{L^{2}(\Omega)}\|\zeta\|_{W_{0}^{1, \lambda}(\Omega)} .
$$

This means that

$$
\left\|w_{n-1}^{-1} \Delta \vartheta_{n}\right\|_{W^{-1, \mu}(\Omega)} \leq c\left\|\nabla \vartheta_{n}\right\|_{L^{2}(\Omega)} \text { for all } \mu=\lambda /(\lambda-1) \in(1,3 / 2),
$$

which along with (3.10)-(3.11) yields

$$
\begin{aligned}
\left\|v_{n}-v_{n-1}\right\|_{W^{-1, \mu}(\Omega)} & \leq c \tau\left\|\nabla \vartheta_{n}\right\|_{L^{2}(\Omega)}+\left\|R_{n}\right\|_{L^{\mu}(\Omega)} \\
& \leq c \tau\left\|\vartheta_{n}\right\|_{W^{1,2}(\Omega)}+c \tau\left\|v_{n-1}\right\|_{L^{\mu}(\Omega)} .
\end{aligned}
$$

Thus we get

$$
\begin{aligned}
& \sum_{n=1}^{N}\left\|v_{n}-v_{n-1}\right\|_{W^{-1, \mu}(\Omega)} \leq c \tau \sum_{n=1}^{N}\left(\left\|\vartheta_{n}\right\|_{W^{1,2}(\Omega)}+\left\|v_{n-1}\right\|_{L^{\mu}(\Omega)}\right) \\
& \leq c \int_{0}^{T}\left(\left\|\vartheta_{N}(t)\right\|_{W^{1,2}(\Omega)}+\left\|v_{N}(t-\tau)\right\|_{L^{\mu}(\Omega)}\right) d t \leq c .
\end{aligned}
$$

It follows that the total variation of the piecewise constant function $v_{N}$ : $[0, T] \rightarrow W^{-1, \mu}(\Omega)$ is bounded by a constant $c$ independent of $N$. Thus

$$
\int_{0}^{T-h}\left\|v_{N}(t+h)-v_{N}(t)\right\|_{W^{-1, \mu}(\Omega)} d t \leq c h \text { for } 0<h<T .
$$

On the other hand, estimate (2.21) yields

$$
\int_{0}^{T-h}\left\|v_{N}(t)\right\|_{W^{1,2}(\Omega)} \leq c h \text { for } 0<h<T .
$$

As the embedding $W^{1,2}(\Omega) \hookrightarrow L^{\mu}(\Omega) \hookrightarrow W^{-1, \mu}(\Omega)$ is compact, Theorem 5 in [24] implies that the sequence $v_{N}$ is relatively compact in $L^{1}\left(0, T ; L^{\mu}(\Omega)\right)$. Hence it is relatively compact in measure. On the other hand, in view of Proposition 2.1 this sequence is bounded in $L^{r}\left(0, T ; L^{p}(\Omega)\right)$ for all $r$ and $p$ satisfying (2.20). Since the set of admissible $r$ and $p$ is open, we conclude that the sequence $v_{N}$ is relatively compact in $L^{r}\left(0, T ; L^{p}(\Omega)\right)$. This completes the proof of Theorem 3.1. 
Proof of Theorem 3.2. Since $L^{p}(\Omega)$ is separable, the piecewise constant mappings $v_{N}:(0, T) \rightarrow L^{p}(\Omega)$ are strongly measurable on $(0, T)$. On the other hand, they converge strongly to $v$ in $L^{r}\left(0, T ; L^{p}(\Omega)\right)$, Hence $v_{N}$ converges to $v$ in measure in $(0, T)$, and the sequence $v_{N}$ meets all requirements of the Egoroff Theorem. We conclude that for every $\eta>0$ there is a compact set $\mathcal{T}_{\eta}$ satisfying (i) and (ii). Item (iii) obviously follows from (3.3).

In order to prove (iv) notice that in view of (2.6) and (2.9) we have

$$
\vartheta_{N}(t)=v_{N}(t)+W\left(\bar{w}_{N}(t)^{-1} D \mathbf{u}_{N}(t)\right),
$$

where $\bar{w}_{N}(t)$ is defined by (2.9). It follows from (3.3) that

$$
\left\|\bar{w}_{N}-w_{N}\right\|_{C\left(0, T ; L^{\alpha}(\Omega)\right)} \rightarrow 0 \text { uniformly in } N \text { as } N \rightarrow \infty .
$$

Now choose a sequence $t_{m} \in \mathcal{T}_{\eta}, m \geq 1$. After passing to a subsequence we may assume that $t_{m} \rightarrow t_{0} \in \mathcal{T}_{\eta}$ as $m \rightarrow \infty$. It follows from (3.3) and (3.15) that $\bar{w}_{N}\left(t_{m}\right) \rightarrow w\left(t_{0}\right)$ in $L^{\alpha}(\Omega)$ as $m, N \rightarrow \infty$. After passing to a subsequence we may assume that

$$
\bar{w}_{N}\left(t_{m}, x\right) \rightarrow w\left(t_{0}, x\right) \text { a.e. in } \Omega \text {. }
$$

Next, it follows from (2.17) that the sequence $\mathbf{u}_{n}\left(t_{m}\right)$ is uniformly bounded in $W^{2,6}(\Omega)$. Recall that the embedding $W^{2,6}(\Omega) \hookrightarrow C^{1}(\Omega)$ is compact. Hence, after passing to a subsequence we may assume that $D \mathbf{u}_{N}\left(t_{m}\right)$ converges uniformly in $\Omega$. Recalling (3.15) we deduce that $W\left(\bar{w}_{N}\left(t_{m}\right)^{-1} D \mathbf{u}_{N}\left(t_{m}\right)\right)$ converges in measure in $\Omega$. Since the functions $W\left(\bar{w}_{N}\left(t_{m}\right)^{-1} D \mathbf{u}_{N}\left(t_{m}\right)\right)$ are bounded, it follows that this sequence converges in $L^{p}(\Omega)$. On the other hand, (ii) implies that $v_{N}\left(t_{m}\right)$ converges to $v\left(t_{0}\right)$ in $L^{p}(\Omega)$. From this and (3.14) we find that $\vartheta_{N}\left(t_{m}\right)$ converges in $L^{p}(\Omega)$ as $(m, N) \rightarrow \infty$. Hence, every sequence $\vartheta_{N}\left(t_{m}\right)$ contains a subsequence which converges in $L^{p}(\Omega)$. Next, in view of (2.8) and (1.20e),

$$
\begin{aligned}
\varphi_{N} & =-\frac{\varepsilon \bar{w}_{N}^{2}}{2 w_{N}}\left(\frac{\Delta \mathbf{u}_{N}}{\bar{w}_{N}}\right)^{2}-w_{N}^{3} \frac{3 \vartheta_{N}^{2}}{2} \\
& +w_{N}^{3}\left(1+\vartheta_{N}\right)\left\{3 W\left(w_{N}^{-1} D \mathbf{u}_{N}\right)-w_{N}^{-1} W^{\prime}\left(w_{N}^{-1} D \mathbf{u}_{N}\right): D \mathbf{u}_{N}\right\} .
\end{aligned}
$$

Consider now $\varphi_{N}\left(t_{m}\right)$. By (2.18) the sequence $\bar{w}_{N} \Delta \mathbf{u}_{N}\left(t_{m}\right)$ is bounded in $W^{1,2}(\Omega)$. Hence, after passing to a subsequence, we may assume that this sequence converges a.e. in $\Omega$. We have proved that $\bar{w}_{N}\left(t_{m}\right), \vartheta_{n}\left(t_{m}\right)$ and $D \mathbf{u}_{N}\left(t_{m}\right)$ converge a.e. in $\Omega$ as $(N, m) \rightarrow \infty$. Hence $\varphi_{N}\left(t_{m}\right)$ converges a.e. in $\Omega$.

Next, since $w_{N}^{ \pm 1}$ and $D \mathbf{u}_{N}$ are uniformly bounded, relation (3.15) implies that for every $t \in(0, T)$,

$$
\left|\varphi_{N}(t)\right| \leq c+c\left|\Delta \mathbf{u}_{N}(t)\right|^{2}+c\left|\vartheta_{N}(t)\right|^{2}
$$

Notice that in view of $(2.20)$ we have

$$
\left\|\left|\Delta \mathbf{u}_{N}(t)\right|^{2}\right\|_{L^{q}(\Omega)} \leq c\left\|\Delta \mathbf{u}_{N}\right\|_{L^{\infty}\left(0, T ; L^{6}(\Omega)\right)} \leq c
$$

We have already proved that the sequence $\vartheta_{N}\left(t_{m}\right)$ is relatively compact in $L^{p}(\Omega)$. From this and $2 q=p$ we conclude that the sequence $\vartheta_{N}\left(t_{m}\right)^{2}$ is 
relatively compact in $L^{q}(\Omega)$. Recalling (3.18) and (3.19) we conclude that the sequence $\varphi_{N}\left(t_{m}\right)$ is bounded in $L^{q}(\Omega)$ for all $q$ satisfying (2.20). Since this sequence converges in measure in $\Omega$ and the set of admissible $q$ is open, we conclude that $\varphi_{N}\left(t_{m}\right)$ converges strongly in $L^{q}(\Omega)$. Thus we prove that for every $t_{m} \in \mathcal{T}_{\eta}$, the sequence $\varphi_{N}\left(t_{m}\right)$ contains a subsequence which converges in $L^{q}(\Omega)$. Hence the set $\mathfrak{T}(\eta)$ is relatively compact in $L^{p}(\Omega) \times L^{q}(\Omega)$. This completes the proof of Theorem 3.2.

\section{Marginal function. Energy dissipation inequalities}

In this section we deduce the energy dissipation inequalities which play a crucial role in the further analysis. In Sections 2 and 3 we have built the sequence of approximate solutions $\mathbf{u}_{N}, \vartheta_{N}, w_{N}$, and $v_{N}$ to problem (1.20) and investigated their properties. In particular, we have proved the strong convergence of the evolutionary variables $v_{N}$ and $w_{N}$. Now we start a long way in order to prove the convergence of $\vartheta_{N}$ and $\varphi_{N}$. Our tool is the monotonicity method, which is based on a careful analysis of the energy dissipation inequality and works well for problems with a convex free energy functional. In our case the main difficulty is that the free energy density is a nonconvex function of the displacement vector field $\mathbf{u}$. However, it is a concave function of the temperature $\vartheta$. Moreover, the right hand side of equation (1.20c) for the growth factor $w$ is a monotone function of the trace $\varphi$ of the material Eshelby tensor. The idea is to eliminate the displacement vector field and to focus on the sequences $\vartheta_{N}$ and $\varphi_{N}$. The key observation is the following.

Substituting the approximate solution into expressions (1.28)-(1.29) for the internal energy we get the approximate value of the total internal energy $\mathcal{E}_{N}$ as a real valued function of the time variable. Since the free energy and the internal energy depend on the displacement vector field $\mathbf{u}$, it is hard to expect that the sequence $\mathcal{E}_{N}$ converges for a fixed $t$. It is a remarkable fact of the theory is that the sequence of internal energies converges almost everywhere on $(0, T)$ and its limit can be expressed in terms of a marginal function depending only on the evolutionary variables $v$ and $w$. This fact immediately leads to the desired energy dissipation inequality. Recall Definition 1.2 for the functional $\mathcal{H}$ and the marginal function $\mathbf{M}$. Now we are in a position to formulate the first main result of this section.

Theorem 4.1 Let all conditions of Theorem 3.1 be satisfied and $v, w$ be the limits of $v_{N}$ and $w_{N}$ defined by Theorem 3.1. Then

$$
\mathcal{H}\left(\mathbf{u}_{N}(t), v_{N}(t), \bar{w}_{N}(t)\right) \rightarrow \mathbf{M}(v(t), w(t)) \text { as } N \rightarrow \infty \text { for a.e. } t \in(0, T) .
$$

Moreover,

$$
\begin{gathered}
\mathbf{M}\left(v\left(t_{0}\right), w\left(t_{0}\right)\right)-\mathbf{M}\left(v\left(t_{1}\right), w\left(t_{1}\right)\right)+\limsup _{N \rightarrow \infty}\left\{\int_{t_{1}+\tau}^{t_{0}} \Pi_{1}\left(H\left(\varphi_{N}\right), \varphi_{N}\right) d s\right. \\
\left.+\frac{1}{2} \int_{t_{1}+\tau}^{t_{0}} \Pi_{0}\left(\vartheta_{N}, \vartheta_{N}\right) d s+\frac{1}{2} \int_{t_{1}+\tau}^{t_{0}-\tau} \Pi\left(\bar{\vartheta}_{N}, \bar{\vartheta}_{N}\right) d s\right\} \leq 0 .
\end{gathered}
$$


for a.e. $0<t_{1}<t_{0}<T$. Here the energy dissipation rate $\Pi$ is given by (1.33), the function $\bar{\vartheta}_{N}$ is given by (2.9), (2.10).

Inequality (4.2) estimates $\mathbf{M}\left(t_{0}\right)-\mathbf{M}\left(t_{1}\right)$ from above. Our next task is to estimate this difference from below. We will thus obtain an estimate which is complementary to the energy dissipation inequality. Such estimates are essential ingredients of the monotonicity method.

In order to formulate the corresponding result we introduce some notation. In view of Proposition 2.1 after passing to a subsequence we may assume that there are functions

$$
\begin{gathered}
\vartheta^{*} \in L^{2}\left(0, T ; W^{1,2}(\Omega)\right) \cap L^{\infty}\left(0, T ; L^{2}(\Omega)\right), \\
\varphi^{*} \in L^{s}\left(0, T ; L^{q}(\Omega)\right), \quad H^{*} \in L^{\infty}(\Omega \times(0, T))
\end{gathered}
$$

such that

$$
\begin{gathered}
\vartheta_{N} \rightarrow \vartheta^{*} \text { weakly in } L^{r}\left(0, T ; L^{p}(\Omega)\right) \text { and in } L^{2}\left(0, T ; W^{1,2}(\Omega)\right), \\
\varphi_{N} \rightarrow \varphi^{*} \text { weakly in } L^{s}\left(0, T ; L^{q}(\Omega)\right), \\
H\left(\varphi_{N}\right) \rightarrow H^{*} \text { star weakly in } L^{\infty}(\Omega \times(0, T)) .
\end{gathered}
$$

Here $(r, p)$ and $(s, q)$ are arbitrary exponents satisfying (2.20). Since the spaces $W^{1,2}(\Omega)$ and $L^{\alpha}(\Omega), 1 \leq \alpha<\infty$, are separable, the mappings $v, \vartheta^{*}$ : $(0, T) \rightarrow W^{1,2}(\Omega)$ and $H^{*}:(0, T) \rightarrow L^{\alpha}(\Omega)$ are strongly measurable. It follows that there exists a set $\mathcal{L}$ of full measure in $(0, T)$ such that for all $t_{0}, t_{1} \in \mathcal{L}$ we have

$$
\begin{aligned}
\frac{1}{t_{0}-t_{1}} \int_{t_{1}}^{t_{0}}\left(\| v\left(t_{0}\right)-\right. & v(s)\left\|_{W^{1,2}(\Omega)}+\right\| \vartheta^{*}\left(t_{0}\right)-\vartheta^{*}(s) \|_{W^{1,2}(\Omega)} \\
& \left.+\left\|H^{*}\left(t_{0}\right)-H^{*}(s)\right\|_{L^{\alpha}(\Omega)}\right) d s \rightarrow 0 \text { as } t \nearrow t_{0} .
\end{aligned}
$$

For every $\eta>0$, the set $\mathcal{L}$ contains a compact subset $\mathcal{L}_{\eta}$ with meas $([0, T]\rangle$ $\left.\mathcal{L}_{\eta}\right)<\eta / 2$. Next, it follows from the Lusin theorem that there is a compact set $\mathcal{C}_{\eta} \subset[0, T]$ such that meas $\left([0, T] \backslash \mathcal{C}_{\eta}\right)<\eta / 2$ and

$$
\begin{aligned}
& \lim _{t_{1} \rightarrow t_{0}, t_{i} \in \mathcal{C}_{\eta}}\left(\left\|v\left(t_{0}\right)-v\left(t_{1}\right)\right\|_{W^{1,2}(\Omega)}\right.+\left\|\vartheta^{*}\left(t_{0}\right)-\vartheta^{*}\left(t_{1}\right)\right\|_{W^{1,2}(\Omega)} \\
&\left.+\left\|H^{*}\left(t_{0}\right)-H^{*}\left(t_{1}\right)\right\|_{L^{\alpha}(\Omega)}\right)=0 .
\end{aligned}
$$

Theorem 4.2 Let $t_{0}, t_{1} \in \mathcal{L}_{\eta} \cap \mathcal{C}_{\eta}$. Furthermore, assume that

$$
\mathbf{u} \in W^{2,6}(\Omega) \cap\left(\mathcal{W}^{2,2}+\mathbf{h}\right)
$$

is a minimizer of the functional $\mathcal{H}\left(\cdot, v\left(t_{0}\right), w\left(t_{0}\right)\right)$, i.e.,

$$
\mathcal{H}\left(\mathbf{u}, v\left(t_{0}\right), w\left(t_{0}\right)\right)=\mathbf{M}\left(v\left(t_{0}\right), w\left(t_{0}\right)\right) .
$$

and

$$
\begin{aligned}
& \left.\vartheta=v\left(t_{0}\right)-W\left(w\left(t_{0}\right)^{-1} D \mathbf{u}\right)\right) \in W^{1,2}(\Omega) \\
& \varphi=\varphi\left(D^{2} \mathbf{u}, D \mathbf{u}, \vartheta, w\left(t_{0}\right)\right) \in L^{q}(\Omega) .
\end{aligned}
$$


Then

$$
\begin{aligned}
\liminf _{t_{1} \nearrow t_{0}} \frac{1}{t_{0}-t_{1}}\left\{\mathbf{M}\left(v\left(t_{0}\right), w\left(t_{0}\right)\right)-\mathbf{M}\left(v\left(t_{1}\right), w\left(t_{1}\right)\right)\right\} \\
+\Pi_{0}\left(\vartheta, \vartheta^{*}\left(t_{0}\right)\right)+\Pi_{1}\left(\varphi, H^{*}\left(t_{0}\right)\right) \geq 0 .
\end{aligned}
$$

Here the bilinear forms $\Pi_{i}$ are given by (1.33).

The rest of the section is devoted to the proof of Theorems 4.1 and 4.2.

Proof of Theorem 4.1. Proof of (4.1). Recall representations (2.3) and (2.9) of the approximate solution in terms of $\mathbf{u}_{n}, v_{n}, \vartheta_{n}$, and $w_{n}$. Fix $\mathbf{u}$ such that $\mathbf{u}-\mathbf{h} \in \mathcal{W}^{2,2}$ and define $\phi \in W^{1,2}(\Omega)$ as a solution to the variational problem

$$
\mathbf{S}_{n}(\phi, \mathbf{u})=\max _{\vartheta \in W^{1,2}(\Omega)} \mathbf{S}_{n}(\vartheta, \mathbf{u}),
$$

where $\mathbf{S}_{n}$ is given by (2.1). It follows from (2.5) that

$$
\mathbf{S}_{n}\left(\vartheta_{n}, \mathbf{u}_{n}\right) \leq \mathbf{S}_{n}(\phi, \mathbf{u})
$$

Note that

$$
\mathbf{S}_{n}\left(\vartheta_{n}, \mathbf{u}_{n}\right)=\sup _{\vartheta \in W^{1,2}(\Omega)} \mathbf{S}_{n}\left(\vartheta, \mathbf{u}_{n}\right) .
$$

It now follows from Lemma A.1 in the Appendix that

$$
\begin{aligned}
\mathbf{S}_{n}\left(\vartheta_{n}, \mathbf{u}_{n}\right) & =\mathbf{E}\left(\mathbf{u}_{n}, \vartheta_{n}, w_{n-1}\right)-\int_{\Omega} \mathbf{f} \cdot \mathbf{u}_{n} d x+\frac{\tau}{2} \Pi_{0}\left(\vartheta_{n}, \vartheta_{n}\right) \\
& \equiv \mathcal{H}\left(\mathbf{u}_{n}, v_{n}, w_{n-1}\right)+\frac{\tau}{2} \Pi_{0}\left(\vartheta_{n}, \vartheta_{n}\right) .
\end{aligned}
$$

Next, expressions (1.13a) and (2.1) for $\Psi_{g}$ and $\mathbf{S}_{n}$ imply

$$
\begin{array}{r}
\mathbf{S}_{n}(\phi, \mathbf{u})=\int_{\Omega} \Psi_{g}\left(D^{2} \mathbf{u}, D \mathbf{u}, \phi, w_{n-1}\right) d x+\int_{\Omega}\left(w_{n-2}^{3} v_{n-1} \phi-f \mathbf{u}\right) d x \\
-\frac{\tau}{2} \Pi_{0}(\phi, \phi) .
\end{array}
$$

Now set

$$
\Theta=\Theta\left(D \mathbf{u}, v_{n}, w_{n-1}\right) \equiv v_{n}+W\left(w_{n-1} D \mathbf{u}\right) .
$$

Obviously we have

$$
V\left(D \mathbf{u}, \Theta, w_{n-1}\right) \equiv \Theta-W\left(w_{n-1} D \mathbf{u}\right)=v_{n},
$$

and

$$
\frac{\partial}{\partial \Theta} \Psi_{g}\left(D^{2} \mathbf{u}, D \mathbf{u}, \Theta, w_{n-1}\right) \equiv-w_{n-1}^{3} V\left(D \mathbf{u}, \Theta, w_{n-1}\right)=-w_{n-1}^{3} v_{n} .
$$

Since $\Psi_{g}$ is a concave function of the temperature, we have

$$
\begin{aligned}
& \Psi_{g}\left(D^{2} \mathbf{u}, D \mathbf{u}, \phi, w_{n-1}\right) \\
& \quad \leq \Psi_{g}\left(D^{2} \mathbf{u}, D \mathbf{u}, \Theta, w_{n-1}\right)+\frac{\partial}{\partial \Theta} \Psi_{g}\left(D^{2} \mathbf{u}, D \mathbf{u}, \Theta, w_{n-1}\right)(\phi-\Theta) .
\end{aligned}
$$


Substituting this in the right hand side of (4.12) and using (4.15) we arrive at

$$
\begin{array}{r}
\left.\mathbf{S}_{n}(\phi, \mathbf{u}) \leq \int_{\Omega} \Psi_{g}\left(D^{2} \mathbf{u}, D \mathbf{u}, \Theta, w_{n-1}\right)+w_{n-1}^{3} v_{n} \Theta\right) d x-\int_{\Omega} f \cdot \mathbf{u} d x \\
+\int_{\Omega}\left(w_{n-2}^{3} v_{n-1}-w_{n-1}^{3} v_{n}\right) \phi d x-\frac{\tau}{2} \Pi_{0}(\phi, \phi) .
\end{array}
$$

Next, (4.13) and expression (1.28) for the density of the internal energy give the identity

$$
\Psi_{g}\left(D^{2} \mathbf{u}, D \mathbf{u}, \Theta, w_{n-1}\right)+w_{n-1}^{3} v_{n} \Theta=\mathcal{E}\left(D^{2} \mathbf{u}, D \mathbf{u}, v_{n}, w_{n-1}\right),
$$

which along with (1.30) implies

$$
\int_{\Omega}\left(\Psi_{g}\left(D^{2} \mathbf{u}, D \mathbf{u}, \Theta, w_{n-1}\right)+w_{n-1}^{3} v_{n} \Theta-\mathbf{f} \cdot \mathbf{u}\right) d x=\mathcal{H}\left(\mathbf{u}, v_{n}, w_{n-1}\right)
$$

Multiplying both sides of (3.9) by $\phi$ and integrating the result over $\Omega$ we obtain

$$
\int_{\Omega}\left(w_{n-2}^{3} v_{n-1}-w_{n-1}^{3} v_{n}\right) \phi d x=\tau \Pi_{0}\left(\vartheta_{n}, \phi\right) .
$$

Substituting (4.17) and (4.18) into (4.16) we obtain

$$
\mathbf{S}_{n}(\mathbf{u}, \phi) \leq \mathcal{H}\left(\mathbf{u}, v_{n}, w_{n-1}\right)+\tau \Pi_{0}\left(\vartheta_{n}, \phi\right)-\frac{\tau}{2} \Pi_{0}(\phi, \phi) .
$$

Combining (4.10) with (4.11) and (4.19) we arrive at

$$
\mathcal{H}\left(\mathbf{u}_{n}, v_{n}, w_{n-1}\right) \leq \mathcal{H}\left(\mathbf{u}, v_{n}, w_{n-1}\right)-\frac{\tau}{2} \Pi_{0}\left(\vartheta_{n}-\phi, \vartheta_{n}-\phi\right)
$$

for all integers $n \in[1, N]$. Recalling the definition (2.2) of $\mathbf{u}_{N}$ and $v_{N}$ and the definition $(2.9)$ of $\bar{w}_{N}$ we deduce that

$$
\mathcal{H}\left(\mathbf{u}_{N}(t), v_{N}(t), \bar{w}_{N}(t)\right) \leq \mathcal{H}\left(\mathbf{u}, v_{N}(t), \bar{w}_{N}(t)\right)
$$

for all $t \in(0, T)$ and all $\mathbf{u} \in \mathcal{W}^{2,2}+\mathbf{h}$. By (3.3) there exists a set $\mathcal{Q}$ of full measure in $[0, T]$ such that $\mathcal{T}_{\eta} \subset \mathcal{Q}$ and for every $t \in \mathcal{Q}$,

$$
v_{N}(t) \rightarrow v(t) \text { in } L^{p}(\Omega), \quad \bar{w}_{N}(t) \rightarrow w(t) \text { in } L^{\alpha}(\Omega) .
$$

Letting $N \rightarrow \infty$ in (4.20), we obtain

$$
\limsup _{N \rightarrow \infty} \mathcal{H}\left(\mathbf{u}_{N}(t), v_{N}(t), \bar{w}_{N}(t)\right) \leq \mathcal{H}(\mathbf{u}, v(t), w(t))
$$

for all $\mathbf{u} \in \mathcal{W}^{2,2}+\mathbf{h}$ which along with the definition of the marginal function $\mathbf{M}$ gives

$$
\limsup _{N \rightarrow \infty} \mathcal{H}\left(\mathbf{u}_{N}(t), v_{N}(t), \bar{w}_{N}(t)\right) \leq \mathbf{M}(v(t), w(t)) \text { for all } t \in \mathcal{Q} .
$$


It remains to prove that

$$
\liminf _{N \rightarrow \infty} \mathcal{H}\left(\mathbf{u}_{N}(t), v_{N}(t), \bar{w}_{N}(t)\right) \geq \mathbf{M}(v(t), w(t)) \text { for all } t \in \mathcal{T}_{\eta}
$$

To this end, we fix $\eta>0$ and $t \in \mathcal{T}_{\eta}$, where $\mathcal{T}_{\eta}$ is given by Theorem 3.2. Next, choose a sequence $N_{k}$ such that

$$
\liminf _{N \rightarrow \infty} \boldsymbol{H}\left(\mathbf{u}_{N}(t), v_{N}(t), \bar{w}_{N}(t)\right)=\lim _{N_{k} \rightarrow \infty} \mathcal{H}\left(\mathbf{u}_{N_{k}}(t), v_{N_{k}}(t), \bar{w}_{N_{k}}(t)\right) .
$$

Since $\mathbf{u}_{N}(t)$ is bounded in $W^{2,6}(\Omega)$, we can assume, after passing to a subsequence, that there is $\mathbf{u}^{*} \in W^{2,6}(\Omega)$ such that

$$
\mathbf{u}_{N_{k}}(t) \rightarrow \mathbf{u}^{*} \text { weakly in } W^{2,6}(\Omega), \mathbf{u}_{N_{k}}(t) \rightarrow \mathbf{u}^{*} \text { strongly in } C^{1}(\bar{\Omega}) .
$$

Next, the inequality $\left|\bar{w}_{N_{k}}(t)-w_{N_{k}}(t)\right| \leq c T / N_{k}$ and relation (3.5) imply

$$
\left\|\bar{w}_{N_{k}}(t)-w(t)\right\|_{L^{\alpha}(\Omega)} \rightarrow 0 \text { as } N_{k} \rightarrow \infty \text { for all } \alpha \in[1, \infty) .
$$

Letting $N_{k} \rightarrow \infty$ and using (3.4) we obtain

$$
\boldsymbol{\mathcal { H }}\left(\mathbf{u}^{*}, v(t), w(t)\right) \leq \lim _{N_{k} \rightarrow \infty} \mathcal{H}\left(\mathbf{u}_{N_{k}}(t), v_{N_{k}}(t), \bar{w}_{N_{k}}(t)\right)
$$

On the other hand,

$$
\mathbf{M}(v(t), w(t)) \leq \mathcal{H}\left(\mathbf{u}^{*}, v(t), w(t)\right),
$$

which yields (4.21). Hence the desired relation (4.21) holds for every $t \in \mathcal{T}_{\eta}$. Letting $\eta \rightarrow 0$ we conclude that it holds for a.e. $t \in(0, T)$. This completes the proof of (4.1).

In order to prove (4.2) it suffices to note that the desired inequality obviously follows from (2.14) and (4.1).

Proof of Theorem 4.2 The proof is based on the following lemma.

Lemma 4.1 Let $\varsigma \in L^{\infty}\left(0, T ; W^{1,2}(\Omega)\right), \partial_{t} \varsigma \in L^{\infty}(\Omega)$ and $\varsigma(t)=0$ in a neighborhood of $T$. Then

$$
\int_{0}^{T} \int_{\Omega}\left(w^{3} v \partial_{t} \varsigma-\nabla \vartheta^{*} \nabla \varsigma\right) d x d t-\int_{0}^{T} \int_{\partial \Omega} \vartheta^{*} \varsigma d s+\int_{\Omega} w_{0}^{3} v_{0} \varsigma(0) d x=0
$$

Moreover,

$$
\begin{aligned}
\int_{\Omega}\left(w\left(t_{0}\right)^{3} v\left(t_{0}\right)-w\left(t_{1}\right)^{3} v\left(t_{1}\right)\right) \eta d x+\int_{t_{1}}^{t_{0}} & \int_{\Omega} \nabla \vartheta^{*} \nabla \eta d x d t \\
& +\int_{t_{1}}^{t_{0}} \int_{\partial \Omega} \vartheta^{*} \eta d s d t=0
\end{aligned}
$$

for all $\eta \in W^{1,2}(\Omega)$ and all $t_{0}, t_{1} \in \mathcal{L}_{\eta} \cap \mathcal{C}_{\eta}$. 
Proof The variation of the functional $S_{n}\left(\vartheta, \mathbf{u}_{n}\right)$ at the critical point $\vartheta=\vartheta_{n}$ leads to the equality

$$
\frac{1}{\tau}\left\{\bar{w}_{N}^{3}(t) v_{N}(t)-\bar{w}_{N}^{3}(t-\tau) v_{N}(t-\tau)\right\}-\Delta \vartheta_{N}(t)=0
$$

for $t \in(0, T)$. Notice that

$$
w_{N}(t-\tau)=w_{-1}=w_{0}, \quad v_{N}(t-\tau)=v_{0} \quad \text { for } t \in(0, \tau] .
$$

Multiplying both sides of (4.24) by $\varsigma$ and integrating the result over $\Omega \times(0, T)$, we obtain

$$
\begin{aligned}
\int_{0}^{T} \int_{\Omega}\left\{w_{N}(t)^{3} v_{n}(t) \frac{\varsigma(t+\tau)-\varsigma(t)}{\tau}-\nabla \vartheta^{*} \nabla \varsigma\right\} d x d t \\
\\
\quad-\int_{0}^{T} \int_{\partial \Omega} \vartheta^{*} \varsigma d s+\int_{\Omega} w_{0}^{3} v_{0} \varsigma(0) d x=0 .
\end{aligned}
$$

Letting $N \rightarrow \infty$ and using (3.3) and (4.4) we arrive at (4.22). Next choose $\eta \in W_{0}^{1,2}(\Omega)$ and $t_{i} \in \mathcal{L}_{\eta} \cap \mathcal{C}_{\eta}$. Then choose a compactly supported continuous function $\zeta$ such that

$$
\zeta=1 \text { for } t \in\left(t_{1}, t_{0}-\delta\right), \quad \zeta=0 \text { for } t \in\left(-\infty, t_{1}-\delta\right] \cup\left[t_{0}, \infty\right),
$$

and $\zeta$ is linear on the intervals $\left(t_{1}-\delta, t_{1}\right)$ and $\left(t_{0}-\delta, t_{0}\right)$. Substituting $\varsigma=\zeta \eta$ into (4.22), letting $\delta \rightarrow 0$, and using (4.5) we obtain (4.23).

Let us turn to the proof of Theorem 4.2. We assume that $t_{1}, t_{0} \in \mathcal{L}_{\eta} \cap \mathcal{C}_{\eta}$. By abuse of notation we will write $v_{i}$ and $w_{i}$ instead of $v\left(t_{i}\right)$ and $w\left(t_{i}\right)$. Recall relation (4.7). By the definition of the marginal function, we have $\mathbf{M}\left(v_{1}, w_{1}\right) \leq \mathcal{H}\left(\mathbf{u}, v_{1}, w_{1}\right)$, which leads to

$$
\begin{aligned}
\mathbf{M}\left(v_{0}, w_{0}\right)-\mathbf{M}\left(v_{1}, w_{1}\right) & \geq \mathcal{H}\left(\mathbf{u}, v_{0}, w_{0}\right)-\mathcal{H}\left(\mathbf{u}, v_{1}, w_{1}\right) \\
& =\mathcal{E}\left(\mathbf{u}, v_{0}, w_{0}\right)-\mathcal{E}\left(\mathbf{u}, v_{1}, w_{1}\right) .
\end{aligned}
$$

Here the total internal energy functional $\mathcal{E}$ has the integral representation by (1.29) with the integrand $\mathcal{E}$ given by (1.28). The Taylor formula implies

$$
\begin{gathered}
\mathcal{E}\left(D^{2} \mathbf{u}, D \mathbf{u}, v_{0}, w_{0}\right)-\mathcal{E}\left(D^{2} \mathbf{u}, D \mathbf{u}, v_{1}, w_{1}\right)=\partial_{v} \mathcal{E}\left(D^{2} \mathbf{u}, D \mathbf{u}, v_{0}, w_{0}\right)\left(v_{0}-v_{1}\right) \\
+\partial_{w} \mathcal{E}\left(D^{2} \mathbf{u}, D \mathbf{u}, v_{0}, w_{0}\right)\left(w_{0}-w_{1}\right)+R_{1}+R_{2}+R_{3}
\end{gathered}
$$

where

$$
\begin{aligned}
& R_{1}=-\left(w_{0}-w_{1}\right)^{2} \frac{1}{2} \int_{0}^{1} \partial_{w}^{2} \mathcal{E}\left(D^{2} \mathbf{u}, D \mathbf{u}, v_{0}, \lambda w_{1}+(1-\lambda) w_{0}\right) d \lambda \\
& R_{2}=-\left(w_{0}-w_{1}\right)\left(v_{0}-v_{1}\right) \int_{0}^{1} \partial_{v} \partial_{w} \mathcal{E}\left(D^{2} \mathbf{u}, D \mathbf{u}, v_{0}, \lambda w_{1}+(1-\lambda) w_{0}\right) d \lambda \\
& R_{3}=-\left(v_{0}-v_{1}\right)^{2} \frac{1}{2} \int_{0}^{1} \partial_{v}^{2} \mathcal{E}\left(D^{2} \mathbf{u}, D \mathbf{u}, \lambda v_{1}+(1-\lambda) v_{0}, w_{1}\right) d \lambda
\end{aligned}
$$

Now the task is to let $t_{1} \rightarrow t_{0}$ in expansion (4.26). Our considerations are based on the following lemma. 
Lemma 4.2 Let $\eta \in W^{1,2}(\Omega)$ and $g \in L^{\beta}(\Omega), \beta>1$. Then

$$
\begin{array}{r}
\frac{1}{t_{0}-t_{1}} \int_{\Omega} \eta\left(w\left(t_{0}\right)^{3} v\left(t_{0}\right)-w\left(t_{1}\right)^{3} v\left(t_{1}\right)\right) d x+\int_{\Omega} \nabla \vartheta^{*}\left(t_{0}\right) \nabla \eta d x \\
+\int_{\partial \Omega} \vartheta^{*}\left(t_{0}\right) \eta d s \rightarrow 0 \text { as } t_{1} \rightarrow t_{0},
\end{array}
$$

and

$$
\frac{1}{t_{0}-t_{1}} \int_{\Omega} g(x)\left(w\left(t_{0}\right)-w\left(t_{1}\right)\right) d x+\int_{\Omega} g H^{*}\left(t_{0}\right) w\left(t_{0}\right) d x \rightarrow 0 \quad \text { as } t_{1} \rightarrow t_{0} .
$$

Proof In view of (4.23), we have

$$
\begin{aligned}
\frac{1}{t_{0}-t_{1}} \int_{\Omega} \eta(x) & \left(w\left(t_{0}\right)^{3} v\left(t_{0}\right)-w\left(t_{1}\right)^{3} v\left(t_{1}\right)\right) d x \\
& +\int_{\Omega} \nabla \vartheta^{*}\left(t_{0}\right) \nabla \eta d x d t+\int_{\partial \Omega} \vartheta^{*}\left(t_{0}\right) \eta d s \\
= & \frac{1}{t_{0}-t_{1}} \int_{t_{1}}^{t_{0}}\left\{\int_{\Omega}\left(\nabla \vartheta^{*}\left(t_{0}\right)-\nabla \vartheta^{*}(t)\right) \nabla \eta d x d t\right. \\
& \left.\quad+\int_{\partial \Omega}\left(\vartheta^{*}\left(t_{0}\right)-\vartheta^{*}(t)\right) \eta d s\right\} d t
\end{aligned}
$$

for all $\eta \in W^{1,2}(\Omega)$. Since the embedding $W^{1,2}(\Omega) \hookrightarrow L^{2}(\partial \Omega)$ is bounded, it follows from (4.5) that

$$
\begin{aligned}
& \frac{1}{t_{0}-t_{1}} \int_{t_{1}}^{t_{0}}\left|\int_{\Omega}\left(\nabla \vartheta^{*}\left(t_{0}\right)-\nabla \vartheta^{*}(t)\right) \nabla \eta d x d t+\int_{\partial \Omega}\left(\vartheta^{*}\left(t_{0}\right)-\vartheta^{*}(t)\right) \eta d s\right| d t \\
& \quad \leq c\|\eta\|_{W^{1,2}(\Omega)} \frac{1}{t_{0}-t_{1}} \int_{t_{1}}^{t_{0}} c\left\|\vartheta^{*}\left(t_{0}\right)-\vartheta(t)\right\|_{W^{1,2}(\Omega)} d t \rightarrow 0 \text { as } t_{1} \rightarrow t_{0}
\end{aligned}
$$

which obviously yields (4.28). Next, we have

$$
w_{0}-w_{1}=-\int_{t_{1}}^{t_{0}} H^{*}(s) w(s) d s \text { and }\left|w_{1}-w_{0}\right| \leq c\left(t_{0}-t_{1}\right) .
$$

We thus get

$$
\begin{aligned}
& \frac{1}{t_{0}-t_{1}} \int_{\Omega} g(x)\left(w\left(t_{0}\right)-w\left(t_{1}\right)\right) d x \\
& =-\int_{\Omega} g(x) H^{*}\left(t_{0}\right) w_{0} d x+\frac{1}{t_{0}-t_{1}} \int_{t_{1}}^{t_{0}} \int_{\Omega} g(x)\left(H^{*}\left(t_{0}\right)-H^{*}(t)\right) w_{0} d x d t \\
& \quad+\frac{1}{t_{0}-t_{1}} \int_{t_{1}}^{t_{0}} \int_{\Omega} g(x) H^{*}(t)\left(w_{0}-w(t)\right) d x d t
\end{aligned}
$$


Next, for $\alpha>\beta /(\beta-1)$ we have

$$
\begin{array}{r}
\frac{1}{t_{0}-t_{1}}\left|\int_{t_{1}}^{t_{0}} \int_{\Omega} g(x)\left(H^{*}\left(t_{0}\right)-H^{*}(t)\right) w_{0} d x\right| d t \\
\leq \frac{c\|g\|_{L^{\beta}(\Omega)}}{t_{0}-t_{1}} \int_{t_{1}}^{t_{0}}\left\|H^{*}\left(t_{0}\right)-H^{*}(t)\right\|_{L^{\alpha}(\Omega)} d t \rightarrow 0
\end{array}
$$

as $t_{1} \rightarrow t_{0}$. Now, estimate (4.31) implies

$$
\begin{aligned}
& \frac{1}{t_{0}-t_{1}} \int_{t_{1}}^{t_{0}} \int_{\Omega} \mid g(x) H^{*}(t)\left(w_{0}-\right.w(t)) \mid d x d t \\
& \leq c\left(t_{0}-t_{1}\right) \int_{\Omega}|g(x)| d x \rightarrow 0 \text { as } t_{1} \rightarrow t_{0} .
\end{aligned}
$$

Combining this with (4.32) we arrive at (4.29).

Let us turn to the proof of Theorem 4.2. Our first task is to estimate the quantities $R_{i}$ in expansion (4.26). Since $w_{i}$ and $|D \mathbf{u}|$ are bounded, it follows from formula (1.28) for $\mathcal{E}$ that

$$
\left|\partial_{w}^{2} \mathcal{E}\left(D^{2} \mathbf{u}, D \mathbf{u}, v_{0}, \lambda w_{1}+(1-\lambda) w_{0}\right)\right| \leq c\left(\left|v_{0}\right|^{2}+|\Delta \mathbf{u}|^{2} \mid+1\right) .
$$

From this and inequality (4.31) we obtain

$$
\begin{aligned}
\left(t_{0}-t_{1}\right)^{-1} \int_{\Omega}\left|R_{1}\right| d x & \leq c\left(t_{0}-t_{1}\right) \int_{\Omega}\left(\left|v_{0}\right|^{2}+|\Delta \mathbf{u}|^{2} \mid+1\right) d x \\
& \leq c\left(t_{0}-t_{1}\right) \rightarrow 0 \text { as } t_{1} \rightarrow t_{0} .
\end{aligned}
$$

Let us estimate $R_{2}$. It follows from the boundedness of $w_{i}$ and $D \mathbf{u}$ that

$$
\left|\partial_{v} \partial_{w} \mathcal{E}\left(D^{2} \mathbf{u}, D \mathbf{u}, v_{0}, \lambda w_{1}+(1-\lambda) w_{0}\right)\right| \leq c\left(1+\left|v_{0}\right|\right),
$$

which along with (4.31) yields

$$
\left(t_{0}-t_{1}\right)^{-1}\left|R_{2}\right| \leq c\left(\left|v_{0}\right|+1\right)\left|v_{0}-v_{1}\right| .
$$

Next, (4.6) implies

$$
\left\|v_{0}-v_{1}\right\|_{W^{1,2}(\Omega)} \rightarrow 0, \quad\left\|v_{1}\right\|_{W^{1,2}(\Omega)} \rightarrow\left\|v_{0}\right\|_{W^{1,2}(\Omega)}
$$

as $t_{1} \nearrow t_{0}$. From this we obtain

$$
\left(t_{0}-t_{1}\right)^{-1} \int_{\Omega}\left|R_{2}\right| d x \leq c\left\|v_{0}\right\|_{L^{2}(\Omega)}\left\|v_{0}-v_{1}\right\|_{L^{2}(\Omega)} \rightarrow 0 \text { as } t_{1} \rightarrow t_{0} .
$$

It remains to estimate $R_{3}$. To this end notice that

$$
\partial_{v}^{2} \mathcal{E}\left(D^{2} \mathbf{u}, D \mathbf{u}, \lambda v_{1}+(1-\lambda) v_{0}, w_{1}\right)=w_{1}^{3} .
$$

Thus we get

$$
R_{3}=-\frac{1}{2}\left(v_{0}-v_{1}\right)\left(w_{0}^{3} v_{0}-w_{1}^{3} v_{1}\right)+\frac{1}{2}\left(v_{0}-v_{1}\right)\left(w_{0}^{3}-w_{1}^{3}\right) v_{0}=I_{1}+I_{2} .
$$


We have

$$
\left(t_{0}-t_{1}\right)^{-1}\left|I_{2}\right| \leq c\left(t_{0}-t_{1}\right)^{-1}\left|v_{0}\right|\left|v_{1}-v_{0}\right|\left|w_{1}-w_{0}\right| \leq c\left(1+\left|v_{0}\right|\right)\left|v_{1}-v_{0}\right| .
$$

Hence $I_{2}$ admits estimate (4.36). Arguing as in the proof of (4.38) we obtain

$$
\left(t_{0}-t_{1}\right)^{-1} \int_{\Omega}\left|I_{2}\right| d x \rightarrow 0 \text { as } t_{1} \rightarrow t_{0}
$$

Next, applying Lemma 4.2 with $\eta=v_{0}-v_{1}$ and noting that

$$
\begin{aligned}
\left|\int_{\Omega} \nabla \vartheta^{*}\left(t_{0}\right) \nabla \eta d x d t+\int_{\partial \Omega} \vartheta^{*}\left(t_{0}\right) \eta d s\right| \\
\leq c\left\|\vartheta^{*}\left(t_{0}\right)\right\|_{W^{1,2}(\Omega)}\left\|v_{0}-v_{1}\right\|_{W^{1,2}(\Omega)} \rightarrow 0
\end{aligned}
$$

as $t_{1} \rightarrow t_{0}$, we obtain

$$
\left(t_{0}-t_{1}\right)^{-1} \int_{\Omega}\left|I_{1}\right| d x \rightarrow 0 \text { as } t_{1} \rightarrow t_{0}
$$

and hence

$$
\left(t_{0}-t_{1}\right)^{-1} \int_{\Omega}\left|R_{3}\right| d x \rightarrow 0 \text { as } t_{1} \rightarrow t_{0} .
$$

Thus we have proved that the limits of all second order terms in the Taylor expansion (4.26) equal zero. In order to find the limits of the first order terms, notice that in view of (1.28) we have

$$
\begin{aligned}
\partial_{v} \mathcal{E}\left(D^{2} \mathbf{u}, D \mathbf{u}, v_{0}, w_{0}\right)\left(v_{0}-v_{1}\right) & =w_{0}^{3} \vartheta \\
\partial_{w} \mathcal{E}\left(D^{2} \mathbf{u}, D \mathbf{u}, v_{0}, w_{0}\right)\left(v_{0}-v_{1}\right) & =w_{0}^{-1} \varphi+3 w_{0}^{2} v_{0} \vartheta
\end{aligned}
$$

where $\vartheta$ and $\varphi$ are given by (4.8). It follows that

$$
\begin{aligned}
\partial_{v} \mathcal{E}\left(D^{2} \mathbf{u}, D \mathbf{u}, v_{0}, w_{0}\right)\left(v_{0}-v_{1}\right)+\partial_{w} \mathcal{E}\left(D^{2} \mathbf{u}, D \mathbf{u}, v_{0}, w_{0}\right)\left(w_{0}-w_{1}\right) \\
=\left(w_{0}^{3} v_{0}-w_{1}^{3} v_{1}\right) \vartheta+w_{0}^{-1}\left(w_{0}-w_{1}\right) \varphi+\left(w_{1}^{3}-w_{0}^{3}\right)\left(v_{1}-v_{0}\right) \vartheta \\
\quad+v_{0}\left(w_{1}^{3}-w_{0}^{3}+3 w_{0}^{2}\left(w_{0}-w_{1}\right)\right) \vartheta .
\end{aligned}
$$

Now set $\eta=\vartheta \in W^{1,2}(\Omega)$ and $g=w_{0}^{-1} \varphi \in L^{q}(\Omega)$. Applying Lemma 4.2 we obtain

$$
\begin{aligned}
& \frac{1}{t_{0}-t_{1}} \int_{\Omega}\left(\left(w_{0}^{3} v_{0}-w_{1}^{3} v_{1}\right) \vartheta+w_{0}^{-1}\left(w_{0}-w_{1}\right) \varphi\right) d x \rightarrow \\
& -\int_{\Omega} \nabla \vartheta^{*}\left(t_{0}\right) \nabla \vartheta d x-\int_{\partial \Omega} \vartheta^{*}\left(t_{0}\right) \vartheta d s-\int_{\Omega} \varphi H^{*}\left(t_{0}\right) d x \text { as } t_{1} \rightarrow t_{0} .
\end{aligned}
$$

Next, (4.31) and (4.37) imply

$$
\begin{aligned}
\frac{1}{t_{0}-t_{1}} \int_{\Omega} \mid\left(w_{1}^{3}-\right. & \left.w_{0}^{3}\right)\left(v_{1}-v_{0}\right) \vartheta\left|d x \leq c \int_{\Omega}\right|\left(v_{1}-v_{0}\right) \vartheta \mid d x \\
& \leq c\|\vartheta\|_{W^{1,2}(\Omega)}\left\|v_{0}-v_{1}\right\|_{W^{1,2}(\Omega)} \rightarrow 0 \text { as } t_{1} \rightarrow t_{0} .
\end{aligned}
$$


Finally, we apply estimate (4.31) to obtain

$$
\begin{aligned}
& \frac{1}{t_{0}-t_{1}} \int_{\Omega}\left|v_{0}\left(w_{1}^{3}-w_{0}^{3}+3 w_{0}^{2}\left(w_{0}-w_{1}\right)\right) \vartheta\right| d x \leq \\
& \quad \frac{c}{t_{0}-t_{1}} \int_{\Omega}\left|\vartheta v_{0}\right|\left(w_{1}-w_{0}\right)^{2} d x \leq c\left(t_{0}-t_{1}\right) \rightarrow 0 \text { as } t_{1} \rightarrow t_{0} .
\end{aligned}
$$

Combining (4.41)-(4.43) with identity (4.40) we arrive at

$$
\begin{aligned}
& \frac{1}{t_{0}-t_{1}} \int_{\Omega}\left\{\partial_{v} \mathcal{E}\left(D^{2} \mathbf{u}, D \mathbf{u}, v_{0}, w_{0}\right)\left(v_{0}-v_{1}\right)\right. \\
& \left.\quad+\partial_{w} \mathcal{E}\left(D^{2} \mathbf{u}, D \mathbf{u}, v_{0}, w_{0}\right)\left(w_{0}-w_{1}\right)\right\} d x \\
& \rightarrow-\int_{\Omega} \nabla \vartheta^{*}\left(t_{0}\right) \nabla \vartheta d x-\int_{\partial \Omega} \vartheta^{*}\left(t_{0}\right) \vartheta d s-\int_{\Omega} \varphi H^{*}\left(t_{0}\right) d x
\end{aligned}
$$

as $t_{1} \rightarrow t_{0}$. Substituting this relation along with the limiting relations (4.34), (4.38), (4.39) for the second order remainders $R_{i}$ into the Taylor expansion (4.26) we obtain

$$
\begin{aligned}
\frac{1}{t_{0}-t_{1}}\left(\mathcal{E}\left(\mathbf{u}, v_{0}, w_{0}\right)-\mathcal{E}\left(\mathbf{u}, v_{1}, w_{1}\right)\right) \\
\quad \equiv \frac{1}{t_{0}-t_{1}} \int_{\Omega}\left\{\mathcal{E}\left(D^{2} \mathbf{u}, D \mathbf{u}, v_{0}, w_{0}\right)-\mathcal{E}\left(D^{2} \mathbf{u} D \mathbf{u}, v_{1}, w_{1}\right)\right\} d x \\
\rightarrow-\int_{\Omega} \nabla \vartheta^{*}\left(t_{0}\right) \nabla \vartheta d x-\int_{\partial \Omega} \vartheta^{*}\left(t_{0}\right) \vartheta d s-\int_{\Omega} \varphi H^{*}\left(t_{0}\right) w_{0} d x
\end{aligned}
$$

as $t_{1} \rightarrow t_{0}$. This result along with (4.25) implies the desired relation (4.9).

\section{Sliced measures in Banach spaces}

In this section we develop a theory of sliced measures in Banach spaces. Using this theory we will prove the strong convergence of the sequences $\vartheta_{N}$ and $\varphi_{N}$. For technical reasons, it is convenient to introduce the following notation. Fix exponents $s, q$ and $r, p$ satisfying relations (2.20) and set

$$
X=L^{p}(\Omega) \times L^{q}(\Omega) .
$$

Further we will denote by $\boldsymbol{\omega}_{N}$ and $\boldsymbol{\omega}^{*}$ the couples

$$
\boldsymbol{\omega}_{N}=\left(\vartheta_{N}, \varphi_{N}\right), \quad \boldsymbol{\omega}^{*}=\left(\vartheta^{*}, \varphi^{*}\right)
$$

where the approximate solutions $\vartheta_{N}, \varphi_{N}$ are defined by Theorem 2.1, and the weak limits $\vartheta^{*}, \varphi^{*}$ are given by (4.4). Next, recall the definitions of the compact set $\mathcal{T}_{\eta}$ in Theorem 3.2 and of the compact sets $\mathcal{L}_{\eta}, \mathcal{C}_{\eta}$ in Theorem 4.2. Choose $\eta>0$ and set

$$
\mathcal{F}_{\eta}=\mathcal{T}_{\eta} \cap \mathcal{C}_{\eta} \cap \mathcal{L}_{\eta}, \quad \mathfrak{F}_{\eta}=\left\{\boldsymbol{\omega}_{N}(t): t \in \mathcal{F}_{\eta}, N \geq 1\right\}, \quad \Sigma_{\eta}=\mathrm{cl} \mathfrak{F}_{\eta} .
$$

In view of Theorem 3.2 the set $\mathfrak{F}_{\eta}$ is relatively compact in $X$ and the set $\Sigma_{\eta}$ is compact in $X$. The following theorem gives the desired representation for the weak limits of the sequences $\vartheta_{N}$ and $\varphi_{N}$. 
Theorem 5.1 There exists a Borel measure $\nu$ on $\mathcal{F}_{\eta} \times \Sigma_{\eta}$ and a subsequence of $\boldsymbol{\omega}_{N}$, still denoted by $\boldsymbol{\omega}_{N}$, with the following properties. For every $F \in$ $C\left(\mathcal{F}_{\eta} \times \Sigma_{\eta}\right)$ we have

$$
\lim _{N \rightarrow \infty} \int_{\mathcal{F}_{\eta}} \int_{\Omega} F\left(t, \boldsymbol{\omega}_{N}\right) d x d t=\int_{\mathcal{F}_{\eta} \times \Sigma_{\eta}} F(t, \boldsymbol{\omega}) d \nu(t, \boldsymbol{\omega}) .
$$

Moreover, there is a measurable family of Borel probability measures $\mu_{t}$, $t \in \mathcal{F}_{\eta}$, on $\Sigma_{\eta}$ such that

$$
\int_{\mathcal{F}_{\eta} \times \Sigma_{\eta}} F(t, \boldsymbol{\omega}) d \nu(t, \boldsymbol{\omega})=\int_{\mathcal{T}_{\eta}}\left\{\int_{\Sigma_{\eta}} F(t, \boldsymbol{\omega}) d \mu_{t}(\boldsymbol{\omega})\right\} d t .
$$

There is a set $\mathcal{F} \subset \mathcal{F}_{\eta}$ of full measure such that

$$
\lim _{n \rightarrow \infty} \frac{1}{\operatorname{meas}\left(I_{n} \cap \mathcal{F}_{\eta}\right)} \int_{\left(I_{n} \cap \mathcal{F}_{\eta}\right) \times \Sigma_{\eta}} F(\boldsymbol{\omega}) d \nu(\boldsymbol{\omega})=\int_{\Sigma_{\eta}} F(\boldsymbol{\omega}) d \mu_{t_{0}}(\boldsymbol{\omega})
$$

for all $t_{0} \in \mathcal{F}$, for all continuous functions $F: \Sigma_{\eta} \rightarrow \mathbb{R}$, and for all intervals $I_{n}=\left[t_{n}, t_{0}\right]$ such that $t_{n} \rightarrow t_{0}$.

The following theorem specifies the structure of the support of the measure $\mu_{t}$. Recall that, in view of Theorem 2.17 , there is a constant $c_{0}$ independent of $t$ and $N$ such that

$$
\left\|\mathbf{u}_{N}(t)\right\|_{W^{2,6}(\Omega)} \leq c_{0} \text { for all } N \geq 1 \text { and } t \in[0, T] .
$$

Theorem 5.2 There is a set $\mathcal{D}$ of full measure in $\mathcal{F}_{\eta}$ with the following property. For every $t_{0} \in \mathcal{D}$ and $\boldsymbol{\omega}=(\vartheta, \varphi) \in \operatorname{supp} \mu_{t_{0}}$, there is $\mathbf{u} \in W^{2,6}(\Omega)$ such that $\|\mathbf{u}\|_{W^{2,6}(\Omega)} \leq c_{0}$ and

$$
\begin{gathered}
\vartheta=v\left(t_{0}\right)+W\left(w\left(t_{0}\right)^{-1} D \mathbf{u}\right), \quad \varphi=\varphi\left(D^{2} \mathbf{u}, D \mathbf{u}, \vartheta, w\left(t_{0}\right)\right), \\
\mathcal{H}\left(\mathbf{u}, v\left(t_{0}\right), w\left(t_{0}\right)\right)=\mathbf{M}\left(v\left(t_{0}\right), w\left(t_{0}\right)\right) .
\end{gathered}
$$

Here $\varphi\left(D^{2} \mathbf{u}, D \mathbf{u}, \vartheta, w(t)\right)$ is given by (1.20e), and the functionals $\mathcal{H}, \mathbf{M}$ are given by (1.30) and (1.31).

The rest of this section is devoted to the proof of Theorems 5.1 and 5.2.

Proof of Theorem 5.1. Observe that the space $C\left(\mathcal{F}_{\eta} \times \Sigma_{\eta}\right)$ is separable; let $F_{k}, k \geq 1$, be a dense set in it. Applying the diagonal process we may assume that there is a subsequence of $\boldsymbol{\omega}_{N}$, still denoted by $\boldsymbol{\omega}_{N}$, such that the limit

$$
\lim _{N \rightarrow \infty} \int_{\mathcal{F}_{\eta}} \int_{\Omega} F_{k}\left(t, \omega_{N}\right) d x d t=: \bar{F}_{k}
$$

exists for every $k \geq 1$. Since the set $\left\{F_{k}\right\}$ is dense in $C\left(\mathcal{F}_{\eta} \times \Sigma_{\eta}\right)$, the limit

$$
\lim _{N \rightarrow \infty} \int_{\mathcal{T}_{\eta}} \int_{\Omega} F\left(t, \omega_{N}\right) d x d t=: \bar{F}
$$


exists for every $F \in C\left(\mathcal{F}_{\eta} \times \Sigma_{\eta}\right)$. Obviously the quantity $\bar{F}$ linearly depends on $F$ and satisfies

$$
|\bar{F}| \leq\|F\|_{C\left(\mathcal{F}_{\eta} \times \Sigma_{\eta}\right)}, \quad \bar{F} \geq 0 \text { for } F \geq 0 .
$$

Hence the mapping $F \mapsto \bar{F}$ define a continuous functional on $C\left(\mathcal{F}_{\eta} \times \Sigma_{\eta}\right)$. By the Riesz representation theorem, there exists a nonnegative Borel measure $\nu$ on $\mathcal{F}_{\eta} \times \Sigma_{\eta}$ such that

$$
\bar{F}=\int_{\mathcal{F}_{\eta} \times \Sigma_{\eta}} F(t, \boldsymbol{\omega}) d \nu(t, \boldsymbol{\omega}) .
$$

This leads to representation (5.3). If $F=F(t)$ is independent of $\boldsymbol{\omega}$, we have

$$
\int_{\mathcal{F}_{\eta}} F(t) d t=\int_{\mathcal{F}_{\eta} \times \Sigma_{\eta}} F(t) d \nu(t, \boldsymbol{\omega}) .
$$

This means that the projection of the measure $\nu$ on $\mathcal{F}_{\eta}$ coincides with the restriction of the Lebesgue measure to $\mathcal{F}_{\eta}$. Hence we can apply the disintegration theorem (see [2]) to obtain representation (5.4). It remains to note that (5.5) is a standard result of the theory of measure derivatives.

Proof of Theorem 5.2. The proof falls into three steps.

Step 1. Consider the following construction. By (4.1) the piecewise constant functions $\mathcal{H}\left(\mathbf{u}_{N}(t), v_{N}(t), \bar{w}_{N}(t)\right)$ converge to $\mathbf{M}(v(t), w(t))$ as $N \rightarrow \infty$ for a.e. $t \in \mathcal{F}_{\eta}$. Applying the Egoroff and Lusin theorems we conclude that for every $\delta>0$ there is a set $\mathcal{G}_{\delta} \subset \mathcal{F}_{\eta}$ such that meas $\left(\mathcal{F}_{\eta} \backslash \mathcal{G}_{\delta}\right)<\delta$ and

$$
\mathcal{H}\left(\mathbf{u}_{N}, v_{N}, \bar{w}_{N}\right) \rightarrow \mathbf{M}(v, w) \text { in } C\left(\mathcal{G}_{\delta}\right) .
$$

Step 2. Choose $\delta>0$ and let $t_{0}$ be a Lebesgue point of $\mathcal{G}_{\delta}$. Next, choose $\boldsymbol{\omega}_{0}=\left(\vartheta_{0}, \varphi_{0}\right) \in \operatorname{supp} \mu_{t_{0}} \subset X$. Let us prove that there are sequences $N_{k}$ and $t_{k} \in \mathcal{G}_{\delta}$ such that $t_{k} \nearrow t_{0}$ and $N_{k} \rightarrow \infty$ as $k \rightarrow \infty$, and

$$
\boldsymbol{\omega}_{N_{k}}\left(t_{k}\right) \rightarrow \boldsymbol{\omega}_{0} \text { in } X \text { as } k \rightarrow \infty .
$$

In other words, we have to prove that

$$
\liminf _{\max \left\{N^{-1}, t_{0}-t\right\} \rightarrow 0}\left\|\boldsymbol{\omega}_{N}(t)-\boldsymbol{\omega}_{0}\right\|_{X}^{-1}=0
$$

for $t \in \mathcal{G}_{\delta}$ and $t \leq t_{0}$. Suppose that (5.11) is false. Then there are $m>0$ and $\epsilon>0$ such that

$$
\left\|\boldsymbol{\omega}_{N}(t)-\boldsymbol{\omega}_{0}\right\|_{X} \geq \epsilon \text { for } N \geq m, 0 \leq t_{0}-t \leq m^{-1}, t \in \mathcal{G}_{\delta} .
$$

Choose a continuous nonnegative function $g: X \rightarrow \mathbb{R}$ such that

$$
g(\boldsymbol{\omega})=0 \quad \text { for }\left\|\boldsymbol{\omega}-\boldsymbol{\omega}_{0}\right\|_{X} \geq \epsilon \quad \text { and } \quad g(\boldsymbol{\omega})=1 \quad \text { for }\left\|\boldsymbol{\omega}-\boldsymbol{\omega}_{0}\right\|_{X} \leq \epsilon / 2 .
$$

It follows from (5.12) that

$$
g\left(\boldsymbol{\omega}_{N}(t)\right)=0 \text { for } N \geq m, 0 \leq t_{0}-t \leq m^{-1}, t \in \mathcal{G}_{\delta} .
$$


Now choose $n \geq m$ and set $I_{n}=\left[t_{0}-1 / n, t_{0}\right]$. It follows from (5.13) that

$$
g\left(\boldsymbol{\omega}_{N}(t)\right)=0 \text { for } t \in I_{n} \cap \mathcal{G}_{\delta} \text { and } N \geq m .
$$

Noting that $I_{n} \subset\left(I_{n} \backslash \mathcal{G}_{\delta}\right) \cup\left(I_{n} \cap \mathcal{G}_{\delta}\right)$ we obtain

$$
\int_{I_{n} \cap \mathcal{F}_{\eta}} g\left(\boldsymbol{\omega}_{N}(t)\right) d t \leq \int_{\left(I_{n} \backslash \mathcal{G}_{\delta}\right)} g\left(\boldsymbol{\omega}_{N}(t)\right) d t \leq \operatorname{meas}\left(I_{n} \backslash \mathcal{G}_{\delta}\right) \equiv \sigma_{n}
$$

for all sufficiently large $N$. Letting $N \rightarrow \infty$ and recalling (5.3) we arrive at

$$
\int_{\left(I_{n} \cap \mathcal{F}_{\eta}\right) \times \Sigma_{\eta}} g(\boldsymbol{\omega}) d \nu(t, \boldsymbol{\omega})=\lim _{N \rightarrow \infty} \int_{\left(I_{n} \cap \mathcal{F}_{\eta}\right)} g\left(\boldsymbol{\omega}_{N}(t)\right) d t \leq \sigma_{n} .
$$

Since $t_{0}$ is a Lebesgue point of $\mathcal{G}_{\delta}$, we have $\lim _{n \rightarrow \infty} n \sigma_{n}=0$. Combining this with (5.5) we obtain

$$
\int_{\Sigma_{\eta}} g(\boldsymbol{\omega}) d \mu_{t_{0}}(\boldsymbol{\omega})=\lim _{n \rightarrow \infty} n \int_{\left(I_{n} \cap \mathcal{F}_{\eta}\right) \times \Sigma_{\eta}} g(\boldsymbol{\omega}) d \nu(t, \boldsymbol{\omega})=0 .
$$

Since $g$ is positive in a neighborhood of $\boldsymbol{\omega}_{0}$, this equality contradicts the inclusion $\boldsymbol{\omega}_{0} \in \operatorname{supp} \mu_{t_{0}}$, thus proving (5.10).

Step 3. Let $N_{k}$ and $t_{k} \in \mathcal{G}_{\delta}$ satisfy condition (5.10). It follows from definition (2.2), (2.8) of the approximate solution that

$$
\begin{array}{r}
\vartheta_{N_{k}}\left(t_{k}\right)=v_{N_{k}}\left(t_{k}\right)+W\left(\bar{w}_{N_{k}}\left(t_{k}\right)^{-1} D \mathbf{u}_{N_{k}}\left(t_{k}\right)\right), \\
\varphi_{N_{k}}\left(t_{k}\right)=\varphi\left(D^{2} \mathbf{u}_{N_{k}}(t), D \mathbf{u}_{N_{k}}(t), \vartheta_{N_{k}}(t), w_{N_{k}}(t)\right) .
\end{array}
$$

Since $\mathcal{G}_{\delta} \subset \mathcal{T}_{\eta}$, relations (3.4) and (3.5) in Theorem 3.2 imply

$$
v_{N_{k}} \rightarrow v \text { in } C\left(\mathcal{G}_{\delta} ; L^{p}(\Omega)\right), \quad \bar{w}_{N_{k}}^{ \pm 1}, w_{N_{k}}^{ \pm 1} \rightarrow w^{ \pm 1} \quad \text { in } C\left(\mathcal{G}_{\delta} ; L^{\alpha}(\Omega)\right)
$$

for every $\alpha \in[1, \infty)$ and every $p$ satisfying $(2.20)$. In particular, these relations hold for every $p \in[1,6)$. Moreover the mappings $v: \mathcal{G}_{\delta} \rightarrow L^{p}(\Omega)$ and $v: \mathcal{G}_{\delta} \rightarrow L^{\alpha}(\Omega)$ are continuous. It follows that

$$
v_{N_{k}}\left(t_{k}\right) \rightarrow v\left(t_{0}\right) \quad \text { in } L^{p}(\Omega), \quad \bar{w}_{N_{k}}\left(t_{k}\right)^{ \pm 1}, w_{N_{k}}\left(t_{k}\right)^{ \pm 1} \rightarrow w\left(t_{0}\right)^{ \pm 1} \text { in } L^{\alpha}(\Omega) \text {. }
$$

After passing to a subsequence we may assume that

$v_{N_{k}}\left(t_{k}\right) \rightarrow v\left(t_{0}\right), \bar{w}_{N_{k}}\left(t_{k}\right)^{ \pm 1} \rightarrow w\left(t_{0}\right)^{ \pm 1}, w_{N_{k}}\left(t_{k}\right)^{ \pm 1}(t) \rightarrow w\left(t_{0}\right)^{ \pm 1}$ a.e. in $\Omega$.

Next, estimates (2.17) and (2.18) in Theorem 2.2 imply

$$
\left\|\mathbf{u}_{N_{k}}\left(t_{k}\right)\right\|_{W^{2,6}(\Omega)} \leq c_{0}, \quad\left\|\bar{w}_{N_{K}}\left(t_{k}\right)^{-1} \Delta \mathbf{u}_{N_{k}}\left(t_{k}\right)\right\|_{W^{1,2}(\Omega)} \leq c .
$$

Notice that the embeddings $W^{2,6}(\Omega) \hookrightarrow C^{1}(\Omega)$ and $W^{1,2}(\Omega) \hookrightarrow L^{2}(\Omega)$ are compact. Since the functions $\bar{w}_{N_{k}}\left(t_{k}\right)^{ \pm 1}$ are uniformly bounded and converge to $\bar{w}\left(t_{0}\right)^{ \pm 1}$, we can assume after passing to a subsequence that

$$
\begin{aligned}
\Delta \mathbf{u}_{N_{k}}\left(t_{k}\right) \rightarrow \Delta \mathbf{u} \text { weakly in } L^{6}(\Omega), \quad & D \mathbf{u}_{N_{k}}\left(t_{k}\right) \rightarrow D \mathbf{u} \text { in } C(\Omega), \\
& \Delta \mathbf{u}_{N_{k}}\left(t_{k}\right) \rightarrow \Delta \mathbf{u} \text { a.e. in } \Omega
\end{aligned}
$$


for some $\mathbf{u} \in W^{2,6}(\Omega)$ satisfying (5.20). Letting $k \rightarrow \infty$ in identities (5.16) and using relations (5.19) and (5.21) we arrive at

$$
\begin{gathered}
\vartheta_{N_{k}}\left(t_{k}\right) \rightarrow v\left(t_{0}\right)+W\left(w\left(t_{0}\right)^{-1} D \mathbf{u}\right) \equiv \tilde{\vartheta} \text { a.e. in } \Omega, \\
\varphi_{N_{k}}\left(t_{k}\right) \rightarrow \varphi\left(D^{2} \mathbf{u}, D \mathbf{u}, \tilde{\vartheta}, w\left(t_{0}\right)\right) \text { a.e. in } \Omega .
\end{gathered}
$$

On the other hand, relations (5.10) imply

$$
\boldsymbol{\omega}_{N_{k}}\left(t_{k}\right)=\left(\vartheta_{N_{k}}\left(t_{k}\right), \varphi_{N_{k}}\left(t_{k}\right)\right) \rightarrow \boldsymbol{\omega}\left(t_{0}\right)=\left(\vartheta_{0}, \varphi_{0}\right) \text { in } X \text { as } k \rightarrow \infty .
$$

Thus we get $\tilde{\vartheta}=\vartheta_{0}$. It follows from this and (5.22) that

$$
\vartheta_{0}=v\left(t_{0}\right)+W\left(w\left(t_{0}\right)^{-1} D \mathbf{u}\right), \quad \varphi_{0}=\varphi\left(D^{2} \mathbf{u}, D \mathbf{u}, \vartheta_{0}, w\left(t_{0}\right)\right),
$$

which gives the desired relation (5.7). It remains to prove that $\mathbf{u}$ is a minimizer of the functional $\mathcal{H}\left(\cdot, v\left(t_{0}\right), w\left(t_{0}\right)\right)$. Notice that

$$
\begin{aligned}
& \mathcal{H}\left(\mathbf{u}_{N_{k}}\left(t_{k}\right), v_{N_{k}}\left(t_{k}\right), w_{N_{k}}\left(t_{k}\right)\right)= \\
& \int_{\Omega}\left(\frac{1}{2 w_{N_{k}}\left(t_{k}\right)}\left|\Delta \mathbf{u}_{N_{k}}\left(t_{k}\right)\right|^{2}+w_{N_{k}}\left(t_{k}\right)^{3} W\left(w_{N_{k}}\left(t_{k}\right)^{-1} D \mathbf{u}_{N_{k}}\left(t_{k}\right)\right)\right) d x+ \\
& \frac{1}{2} \int_{\Omega}\left(w_{N_{k}}\left(t_{k}\right)^{3}\left(v_{N_{k}}\left(t_{k}\right)+W\left(w_{N_{k}}\left(t_{k}\right)^{-1} D \mathbf{u}_{N_{k}}\left(t_{k}\right)\right)^{2}-\mathbf{f} \cdot \mathbf{u}_{N_{k}}\left(t_{k}\right)\right) d x .\right.
\end{aligned}
$$

Relations (5.17) and (5.21) imply

$$
\begin{array}{r}
\liminf _{N \rightarrow \infty} \int_{\Omega} \frac{1}{w_{N_{k}}\left(t_{k}\right)}\left|\Delta \mathbf{u}_{N_{k}}\left(t_{k}\right)\right|^{2} d x \geq \int_{\Omega} \frac{1}{w\left(t_{0}\right)}|\Delta \mathbf{u}|^{2} d x, \\
\lim _{N \rightarrow \infty} \int_{\Omega}\left(w_{N_{k}}\left(t_{k}\right)\right)^{3}\left(v_{N_{k}}\left(t_{k}\right)+W\left(w_{N_{k}}\left(t_{k}\right)^{-1} D \mathbf{u}_{N_{k}}\left(t_{k}\right)\right)^{2} d x=\right. \\
\int_{\Omega} w\left(t_{0}\right)^{3}\left(v\left(t_{0}\right)+W\left(w\left(t_{0}\right)^{-1} D \mathbf{u}\right)^{2} d x .\right.
\end{array}
$$

From this and (5.17) we conclude that

$$
\begin{aligned}
\mathcal{H}\left(\mathbf{u}, v\left(t_{0}\right), w\left(t_{0}\right)\right) & \leq \liminf _{k \rightarrow \infty} \mathcal{H}\left(\mathbf{u}_{N_{k}}\left(t_{k}\right), v_{N_{k}}\left(t_{k}\right), w_{N_{k}}\left(t_{k}\right)\right) \\
& =\liminf _{k \rightarrow \infty} \mathcal{H}\left(\mathbf{u}_{N_{k}}\left(t_{k}\right), v_{N_{k}}\left(t_{k}\right), \bar{w}_{N_{k}}\left(t_{k}\right)\right) .
\end{aligned}
$$

On the other hand, relations (5.9) yield

$$
\liminf _{k \rightarrow \infty} \mathcal{H}\left(\mathbf{u}_{N_{k}}\left(t_{k}\right), v_{N_{k}}\left(t_{k}\right), \bar{w}_{N_{k}}\left(t_{k}\right)\right)=\mathbf{M}\left(v\left(t_{0}\right), w\left(t_{0}\right)\right) .
$$

Combining these results we arrive at the inequality

$$
\mathcal{H}\left(\mathbf{u}, v\left(t_{0}\right), w\left(t_{0}\right)\right) \leq \mathbf{M}\left(v\left(t_{0}\right), w\left(t_{0}\right)\right) .
$$

Hence $\mathbf{u}$ is a minimizer of $\mathcal{H}\left(\cdot, v\left(t_{0}\right), w\left(t_{0}\right)\right)$ and we have $\mathcal{H}\left(\mathbf{u}, v\left(t_{0}\right), w\left(t_{0}\right)\right)=$ $\mathbf{M}\left(v\left(t_{0}\right), w\left(t_{0}\right)\right)$. It follows that the desired relation (5.8) holds for all $t_{0} \in \mathcal{G}_{\delta}$. Letting $\delta \rightarrow 0$ we conclude that (5.8) is fulfilled for a.e. $t_{0} \in \mathcal{F}_{\eta}$. This completes the proof of Theorem 5.2. 


\section{Strong convergence of temperature and Eshelby tensor}

In this section we employ the results obtained in Sections 4 and 5 in order to prove the strong convergence of sequences $\vartheta_{N}$ and $\varphi_{N}$. This result is given by the following

Theorem 6.1 Let exponents $(r, p)$ and $(s, q)$ satisfy inequalities (2.20) and $\left(\vartheta^{*}, \varphi^{*}\right)$ be defined by (4.4). Then

$$
\vartheta_{N} \rightarrow \vartheta^{*} \text { in } L^{r}\left(0, T ; L^{p}(\Omega)\right), \quad \varphi_{N} \rightarrow \varphi^{*} \text { in } L^{s}\left(0, T ; L^{q}(\Omega)\right) .
$$

For almost every $t \in(0, T)$, there is a function $\mathbf{u} \in \mathcal{W}^{2,2}+\mathbf{h}$ such that $\|\mathbf{u}\|_{W^{2,6}(\Omega)} \leq c_{0}$ and

$$
\begin{gathered}
\vartheta^{*}(t)=v(t)+W\left(w(t)^{-1} D \mathbf{u}\right), \quad \varphi^{*}(t)=\varphi\left(D^{2} \mathbf{u}, D \mathbf{u}, \vartheta^{*}(t), w(t)\right), \\
\mathcal{H}(\mathbf{u}, v(t), w(t))=\mathbf{M}(v(t), w(t)),
\end{gathered}
$$

i.e., $\mathbf{u}$ is a minimizer of the functional $\mathcal{H}(\cdot, v(t), w(t))$.

The rest of the section is devoted to the proof of Theorem 6.1. We split the proof into the sequence of lemmas. First we prove that the dissipation energy rate $\Pi$ given by $(1.32)$ is integrable with respect to the measure $\mu_{t}$. Notice that $\mu_{t}$ is defined on the compact subset $\Sigma_{\eta}$ of space $X=L^{p}(\Omega) \times L^{q}(\Omega)$, while $\Pi$ is defined on the space $W^{1,2}(\Omega) \times L^{q}(\Omega)$. The energy dissipation rate $\Pi$ can be considered as a discontinuous unbounded functional defined on the dense subspace of $X$. However, we intend to prove that $\Pi(\vartheta, \varphi)$ is integrable over the measure $\mu_{t}$. The proof is based on the special approximation of $\Pi$ which is defined as follows. Recall decomposition (1.33) of $\Pi$,

$$
\Pi(\vartheta, \varphi)=\Pi_{0}(\vartheta, \vartheta)+\Pi_{1}(H(\varphi), \varphi),
$$

where the bilinear forms $\Pi_{i}$ are given by

$$
\Pi_{0}(\vartheta, v)=\int_{\Omega} \nabla \vartheta \nabla v d x+\int_{\partial \Omega} \vartheta v d s, \Pi_{1}(\psi, \varphi)=\int_{\Omega} \psi \varphi d x
$$

In view of the general theory of the second order elliptic equations, see [9], the spectral problem

$$
-\Delta \zeta=\lambda \zeta \text { in } \Omega, \quad \partial_{n} \zeta+\zeta=0 \text { on } \partial \Omega
$$

has a countable set of eigenvalues $\lambda_{k}>0, k \geq 1$, and eigenfunctions $\zeta_{k} \in$ $W^{2,2}(\Omega), k \geq 1$. The eigenfunctions form an orthonormal basis in $L^{2}(\Omega)$ and an orthogonal basis in $W^{1,2}(\Omega)$. Every element $\vartheta \in L^{2}(\Omega)$ admits the representation

$$
\vartheta=\sum_{k} \vartheta_{k} \zeta_{k}, \quad \vartheta_{k}=\int_{\Omega} \vartheta \zeta_{k} d x
$$

In particular, the Bessel identity implies the relations

$$
\|\vartheta\|_{L^{2}(\Omega)}^{2}=\sum_{k}\left|\vartheta_{k}\right|^{2}, \quad \Pi_{0}(\vartheta, \vartheta)=\sum_{k} \lambda_{k}\left|\vartheta_{k}\right|^{2} .
$$


Now set

$$
\Pi^{(n)}(\vartheta, \varphi)=\Pi_{0}^{(n)}(\vartheta, \vartheta)+\Pi_{1}(H(\varphi), \varphi)
$$

where

$$
\Pi_{0}^{(n)}(\vartheta, v)=\Pi_{0}\left(P_{n} \vartheta, P_{n} v\right), \quad P_{n} \vartheta=\sum_{k=1}^{n} \vartheta_{k} \zeta_{k} .
$$

For every $v \in W^{1,2}(\Omega)$ and $\psi \in L^{\infty}(\Omega)$ define the linear forms

$$
\begin{array}{r}
\Gamma(\vartheta, \varphi):=\Pi_{0}(\vartheta, v)+\Pi_{1}(\psi, \varphi), \\
\Gamma^{(n)}(\vartheta, \varphi):=\Pi_{0}\left(P_{n} \vartheta, P_{n} v\right)+\Pi_{1}(\psi, \varphi) .
\end{array}
$$

The following Lemma describe the properties of $\Pi$ and $\Gamma$ and their approximations.

Lemma 6.1 The functions $\Pi, \Gamma: W^{1,2}(\Omega) \times L^{1}(\Omega) \rightarrow \mathbb{R}$ and $\Pi^{(n)}, \Gamma^{(n)}$ : $L^{1}(\Omega) \times L^{1}(\Omega) \rightarrow \mathbb{R}$ are continuous. In particular, $\Pi^{(n)}$ and $\Gamma^{(n)}$ are continuous on the Banach space $X$. For every $\boldsymbol{\omega}=(\vartheta, \varphi) \in X$ we have

$$
\begin{gathered}
\Pi^{(n)}(\boldsymbol{\omega}) \nearrow \Pi(\boldsymbol{\omega}) \text { when } \vartheta \in W^{1,2}(\Omega) \text { and } \Pi^{(n)}(\boldsymbol{\omega}) \nearrow \infty \text { otherwise } \\
\Gamma^{(n)}(\boldsymbol{\omega}) \rightarrow \Gamma(\boldsymbol{\omega}) \text { when } \vartheta \in W^{1,2}(\Omega)
\end{gathered}
$$

as $n \rightarrow \infty$.

Proof The continuity of functions $\Pi, \Gamma$ is obvious. The continuity of $\Pi^{(n)}, \Gamma^{(n)}$ : $L^{1}(\Omega) \times L^{1}(\Omega) \rightarrow \mathbb{R}$ obviously follows from the representations

$$
\begin{gathered}
\Pi^{(n)}(\boldsymbol{\omega})=\sum_{k=1}^{n} \lambda_{k}\left(\int_{\Omega} \vartheta \zeta_{k} d x\right)^{2}+\int_{\Omega} H(\varphi) \varphi d x, \\
\Gamma^{(n)}=\int_{\Omega}\left(v^{(n)} \vartheta+\psi \varphi\right) d x, \quad v^{(n)}=\sum_{1}^{n} \lambda_{k} \psi_{k} \zeta_{k} \in C(\Omega) .
\end{gathered}
$$

Since $\Pi_{0}(\vartheta, \vartheta)$ determines the norm in $W^{1,2}(\Omega)$, relation (6.12) obviously follows from representations (6.9) and (6.10). Since $\zeta_{k}$ form the orthogonal basis in $W^{1,2}(\Omega)$, the sequence $P_{n} \vartheta$ converges $\vartheta$ in $W^{1,2}(\Omega)$, which along with (6.11) yields (6.13).

The next Lemma constitutes the differentiability of the marginal function.

Lemma 6.2 There is a set $\mathcal{Q}$ of the full measure in $(0, T)$ with the following properties. For every $t_{0} \in \mathcal{D}$ we have

$$
\frac{1}{t_{0}-t}\left(\mathbf{M}\left(v\left(t_{0}\right), w\left(t_{0}\right)\right)-\mathbf{M}(v(t), w(t)) \rightarrow \mathbf{M}^{\prime}\left(t_{0}\right) \in(-\infty, 0],\right.
$$

as $t \nearrow t_{0}$ and $t \in \mathcal{Q}$. 
Proof Since $\Pi_{0}$ is nonnegative, inequality (4.2) in Theorem 4.1 implies that

$$
\mathbf{M}\left(v\left(t_{0}\right), w\left(t_{0}\right)\right)-\mathbf{M}(v(t), w(t)) \leq 0
$$

for almost all $0<t<t_{0}<T$. In other words, there is a set $\mathcal{Q}_{1}$ of full measure in $[0, T]$ such that this inequality holds true for all $t<t_{0}$ in this set. Hence the function $\mathbf{M}(v(t), w(t))$ decreases on $\mathcal{Q}_{1}$. Obviously, it can be extended to a decreasing function to the whole interval $[0, T]$. Hence there is a set $\mathcal{Q} \subset \mathcal{Q}_{1}$ of full measure in $(0, T)$ such that the extended function has the non-positive finite derivative $M^{\prime}\left(t_{0}\right)$ at every point of $\mathcal{Q}$.

Without loss of generality we can assume that $\mathcal{Q}$ contains the set $\mathcal{D}$ given by Theorem 5.2. To this end, it suffices to replace $\mathcal{D}$ by $\mathcal{D} \cap \mathcal{Q}$. Thus we can assume that the marginal function is differentiable on $\mathcal{D}$. The following lemma constitutes the integrability of the functions $\Pi$ and $\Gamma$ with respect to the measure $\mu_{t}$.

Lemma 6.3 For every $t_{0} \in \mathcal{D}$ and all $v \in W^{1,2}(\Omega), \psi \in L^{\infty}(\Omega)$, the functions

$$
(\vartheta, \varphi) \mapsto \Pi(\vartheta, \varphi) \text { and }(\vartheta, \varphi) \mapsto \Gamma(\vartheta, \varphi)
$$

are integrable with respect to the measure $\mu_{t_{0}}$ given by Theorem 5.1. Moreover, we have

$$
\int_{\Sigma_{\eta}} \Pi(\omega) d \mu_{t_{0}}(\boldsymbol{\omega}) \leq-M^{\prime}\left(t_{0}\right) .
$$

Proof Choose $t_{1}, t_{0} \in \mathcal{D}$ with $0<t_{1}<t_{0}$. Recall definition (2.9) of the function $\bar{\vartheta}_{N}$. Since $0 \leq \Pi_{0}^{(n)} \leq \Pi_{0}$, we have

$$
\begin{aligned}
& \left.\int_{\left(t_{1}+\tau, t_{0}\right) \cap \mathcal{F}_{\eta}} \Pi_{0}^{(n)}\left(\vartheta_{N}, \vartheta_{N}\right)+\int_{\left(t_{1}+\tau, t_{0}-\tau\right) \cap \mathcal{F}_{\eta}} \Pi_{0}^{(n)}\left(\bar{\vartheta}_{N}, \bar{\vartheta}_{N}\right)\right) \\
& \left.\leq \frac{1}{2} \int_{\left(t_{1}+\tau, t_{0}\right)} \Pi_{0}\left(\vartheta_{N}, \vartheta_{N}\right)+\frac{1}{2} \int_{\left(t_{1}+\tau, t_{0}-\tau\right) \cap} \Pi_{0}\left(\bar{\vartheta}_{N}, \bar{\vartheta}_{N}\right)\right) .
\end{aligned}
$$

It follows from this and inequality (4.2) in Theorem 4.1 that

$$
\begin{array}{r}
\mathbf{M}\left(v\left(t_{0}\right), w\left(t_{0}\right)\right)-\mathbf{M}\left(v\left(t_{1}\right), w\left(t_{1}\right)\right)+ \\
\limsup _{N \rightarrow \infty}\left\{\int_{\left(t_{1}+\tau, t_{0}\right) \cap \mathcal{F}_{\eta}} \Pi_{1}\left(H\left(\varphi_{N}\right), \varphi_{N}\right)\right. \\
\quad+\frac{1}{2} \int_{\left(t_{1}+\tau, t_{0}\right) \cap \mathcal{F}_{\eta}} \Pi_{0}^{(n)}\left(\vartheta_{N}, \vartheta_{N}\right) d s+ \\
\left.\frac{1}{2} \int_{\left(t_{1}+\tau, t_{0}-\tau\right) \cap \mathcal{F}_{\eta}} \Pi_{0}^{(n)}\left(\bar{\vartheta}_{N}, \bar{\vartheta}_{N}\right) d s\right\} \leq 0 .
\end{array}
$$

Notice that the quadratic form $\Pi_{0}^{(n)}: L^{2}(\Omega) \times L^{2}(\Omega) \rightarrow \mathbb{R}$ is continuous. Next, estimate (2.13) in Theorem 2.1 implies that

$$
\int_{0}^{t_{0}}\left\|\vartheta_{N}-\bar{\vartheta}_{N}\right\|_{L^{2}(\Omega)}^{2} d t \rightarrow 0 \text { as } N \rightarrow \infty .
$$


In particular, we have

$$
\int_{0}^{t_{0}} \Pi_{0}^{(n)}\left(\vartheta_{N}-\bar{\vartheta}_{N}, \vartheta_{N}-\bar{\vartheta}_{N}\right) d t \rightarrow 0 \text { as } N \rightarrow \infty .
$$

Since the quadratic form $\Pi^{(n)}$ is nonnegative, the Cauchy inequality implies the estimate

$$
\begin{aligned}
\Pi_{0}^{(n)}\left(\bar{\vartheta}_{N}, \bar{\vartheta}_{N}\right)-\Pi_{0}^{(n)}\left(\vartheta_{N}, \vartheta_{N}\right) \leq \\
\delta \Pi_{0}\left(\vartheta_{N}, \vartheta_{N}\right)+\delta^{-1} \Pi_{0}^{(n)}\left(\vartheta_{N}-\bar{\vartheta}_{N}, \vartheta_{N}-\bar{\vartheta}_{N}\right) .
\end{aligned}
$$

It follows from this and energy estimate (2.12) that

$\int_{0}^{t_{0}}\left|\Pi_{0}^{(n)}\left(\bar{\vartheta}_{N}, \bar{\vartheta}_{N}\right)-\Pi_{0}^{(n)}\left(\vartheta_{N}, \vartheta_{N}\right)\right| d t \leq c \delta+\delta^{-1} \int_{0}^{t_{0}} \Pi_{0}^{(n)}\left(\vartheta_{N}-\bar{\vartheta}_{N}, \vartheta_{N}-\bar{\vartheta}_{N}\right)$.

Letting $N \rightarrow \infty$ we obtain

$$
\limsup _{N \rightarrow \infty} \int_{0}^{t_{0}}\left|\Pi_{0}^{(n)}\left(\bar{\vartheta}_{N}, \bar{\vartheta}_{N}\right)-\Pi_{0}^{(n)}\left(\vartheta_{N}, \vartheta_{N}\right)\right| d t \leq c \delta \rightarrow 0 \text { as } \delta \rightarrow 0 .
$$

It follows that

$$
\lim _{N \rightarrow \infty} \int_{\left(t_{1}+\tau, t_{0}-\tau\right) \cap \mathcal{F}_{\eta}}\left(\Pi_{0}^{(n)}\left(\bar{\vartheta}_{N}, \bar{\vartheta}_{N}\right)-\Pi_{0}^{(n)}\left(\vartheta_{N}, \vartheta_{N}\right)\right) d t=0 .
$$

Combining this relation with (6.17) we arrive at the inequality

$$
\begin{aligned}
& \mathbf{M}\left(v\left(t_{0}\right), w\left(t_{0}\right)\right)-\mathbf{M}\left(v\left(t_{1}\right), w\left(t_{1}\right)\right)+\limsup _{N \rightarrow \infty}\left\{\int_{\left(t_{1}+\tau, t_{0}\right) \cap \mathcal{F}_{\eta}} \Pi_{1}\left(H\left(\varphi_{N}\right), \varphi_{N}\right)\right. \\
& \left.+\frac{1}{2} \int_{\left(t_{1}+\tau, t_{0}\right) \cap \mathcal{F}_{\eta}} \Pi_{0}^{(n)}\left(\vartheta_{N}, \vartheta_{N}\right) d s+\frac{1}{2} \int_{\left(t_{1}+\tau, t_{0}-\tau\right) \cap \mathcal{F}_{\eta}} \Pi_{0}^{(n)}\left(\vartheta_{N}, \vartheta_{N}\right) d s\right\} \leq 0 .
\end{aligned}
$$

In view of (5.2) and Theorem 3.2 the functions $\boldsymbol{\omega}_{N}(t), t \in \mathcal{F}_{\eta}$, belong to the set $\mathfrak{F}(\eta)$ which is relatively compact in $X$. Hence for a fixed $n$, the functions $\Pi^{(n)}\left(\vartheta_{N}(t), \varphi_{N}(t)\right)$ are uniformly bounded on $\mathcal{F}_{\eta}$. Hence the functions $\Pi_{0}^{(n)}\left(\vartheta_{N}, \vartheta_{N}\right)(t)$ and $\Pi_{1}\left(H\left(\varphi_{N}\right), \varphi_{N}\right)$ are bounded on $\mathcal{F}_{\eta}$ uniformly in $N$. Since $\tau \rightarrow 0$ as $N \rightarrow \infty$, we have

$$
\begin{aligned}
& \limsup _{N \rightarrow \infty}\left\{\int_{\left(t_{1}+\tau, t_{0}\right) \cap \mathcal{F}_{\eta}} \Pi_{1}\left(H\left(\varphi_{N}\right), \varphi_{N}\right)+\frac{1}{2} \int_{\left(t_{1}+\tau, t_{0}\right) \cap \mathcal{F}_{\eta}} \Pi_{0}^{(n)}\left(\vartheta_{N}, \vartheta_{N}\right) d s\right. \\
& \left.+\frac{1}{2} \int_{\left(t_{1}+\tau, t_{0}-\tau\right) \cap \mathcal{F}_{\eta}} \Pi_{0}^{(n)}\left(\vartheta_{N}, \vartheta_{N}\right) d s\right\}=\limsup _{N \rightarrow \infty} \int_{\left(t_{1}, t_{0}\right) \cap \mathcal{F}_{\eta}} \Pi^{(n)}\left(\vartheta_{N}, \varphi_{N}\right) d t .
\end{aligned}
$$

Here we use the identity

$$
\Pi_{0}^{(n)}\left(\vartheta_{N}, \vartheta_{N}\right)+\Pi_{1}\left(H\left(\varphi_{N}\right), \varphi_{N}\right)=\Pi^{(n)}\left(\vartheta_{N}, \varphi_{N}\right) .
$$


Combining this result with (6.19) we arrive at the inequality

$$
\limsup _{N \rightarrow \infty} \int_{\left(t_{1}, t_{0}\right) \cap \mathcal{F}_{\eta}} \Pi^{(n)}\left(\vartheta_{N}(t), \varphi_{N}(t)\right) d t \leq-\left\{\mathbf{M}\left(v\left(t_{0}\right), w\left(t_{0}\right)\right)-\mathbf{M}\left(v\left(t_{1}\right), w\left(t_{1}\right)\right)\right\} .
$$

In view of Lemma $6.13 \Pi^{(n)}(\boldsymbol{\omega})$ is continuous in $X$. Hence we can apply Theorem 5.1 to obtain

$$
\lim _{N \rightarrow \infty} \int_{\left[t_{1}, t_{0}\right] \cap \mathcal{F}_{\eta}} \Pi^{(n)}\left(\vartheta_{N}, \varphi_{N}\right) d t=\int_{\left[t_{0}, t_{1}\right] \cap \mathcal{F}_{\eta} \times \Sigma_{\eta}} \Pi^{(n)}(\boldsymbol{\omega}) d \nu(\boldsymbol{\omega}),
$$

where $\boldsymbol{\omega}=(\vartheta, \varphi)$. Thus we get

$$
\int_{\left[t_{0}, t_{1}\right] \cap \mathcal{F}_{\eta} \times \Sigma_{\eta}} \Pi^{(n)}(\boldsymbol{\omega}) d \nu \leq-\left\{\mathbf{M}\left(v\left(t_{0}\right), w\left(t_{0}\right)\right)-\mathbf{M}\left(v\left(t_{1}\right), w\left(t_{1}\right)\right)\right\} .
$$

Since $t_{0} \in \mathcal{D} \subset \mathcal{F}$ we can apply the relation (5.5) in Theorem 5.1 to obtain

$$
\lim _{t_{1} \rightarrow t_{0}} \frac{1}{t_{0}-t_{1}} \int_{\left[t_{0}, t_{1}\right] \cap \mathcal{T}_{\eta} \times \Sigma_{\eta}} \Pi^{(n)}(\boldsymbol{\omega}) d \nu=\int_{\Sigma_{\eta}} \Pi^{(n)}(\boldsymbol{\omega}) d \mu_{t_{0}}(\boldsymbol{\omega}) .
$$

On the other hand, Lemma 6.3 yields

$$
\lim _{t_{1} \rightarrow t_{0}} \frac{1}{t_{0}-t_{1}}\left\{\mathbf{M}\left(v\left(t_{0}\right), w\left(t_{0}\right)\right)-\mathbf{M}\left(v\left(t_{1}\right), w\left(t_{1}\right)\right)\right\}=\mathbf{M}^{\prime}\left(t_{0}\right) .
$$

Combining relations (6.21) and (6.22) with inequality (6.20) we obtain

$$
\int_{\Sigma_{\eta}} \Pi^{(n)}(\omega) d \mu_{t_{0}}(\boldsymbol{\omega}) \leq-\mathbf{M}^{\prime}\left(t_{0}\right)
$$

Relation (6.12) in Lemma 6.1 implies that the sequence $\Pi^{(n)}(\boldsymbol{\omega})$ increases and converges to $\Pi(\boldsymbol{\omega})$. Letting $n \rightarrow \infty$ in (6.23) and applying the Fatou theorem we conclude that the function $\Pi$ is integrable with respect to the measure $\mu_{t_{0}}$ and satisfies inequality (6.16).

Let us prove the integrability of $\Gamma$. Choose $v \in W^{1,2}(\Omega)$ and $\psi \in L^{\infty}(\Omega)$. It follows from the Cauchy inequality that

$$
\Pi_{0}(\vartheta, v) \leq \Pi_{0}(\vartheta, \vartheta)^{1 / 2} \Pi_{0}(v, v)^{1 / 2} \leq c \Pi_{0}(\vartheta, \vartheta)^{1 / 2} \leq c \Pi(\vartheta, \varphi)^{1 / 2}
$$

It follows from this and the representation (6.9) that

$$
|\Gamma(\vartheta, \varphi)| \leq c(1+\Pi(\vartheta, \varphi)) .
$$

Hence $\Gamma$ has the integrable majorant. Since $\vartheta \in W^{1,2}(\Omega)$ almost everywhere on the support $\mu_{t_{0}}$, it follows that the continuous functions $\Gamma^{(n)} \rightarrow \Gamma \mu_{t_{0}}$ almost everywhere. Hence the function $\Gamma$ is measurable and integrable withy respect to $\mu_{t_{0}}$.

The following lemma plays a key role in the proof of Theorem 6.1. 
Lemma 6.4 The inequality

$$
\int_{\Sigma_{\eta}} \Pi(\vartheta, \varphi) d \mu_{t_{0}} \leq \int_{\Sigma_{\eta}}\left(\Pi_{0}\left(\vartheta, \vartheta^{*}\left(t_{0}\right)\right)+\Pi_{1}\left(H^{*}\left(t_{0}\right), \varphi\right)\right) d \mu_{t_{0}}
$$

holds true for every $t_{0} \in \mathcal{D}$. Here $\vartheta^{*}$ and $H^{*}$ are the weak limits of $\vartheta_{N}$ and $H\left(\varphi_{N}\right)$ defined by $(4.4)$.

Proof . Choose $t_{0} \in \mathcal{D} \subset \mathcal{F}_{\eta}$. Recall that $\mathcal{F}_{\eta} \in \mathcal{C}_{\eta}$, where $\mathcal{C}_{\eta}$ is given by (4.6). It follows from (4.6) that $\vartheta^{*}\left(t_{0}\right) \in W^{1,2}(\Omega)$. By virtue of Theorem 5.2, every element $\boldsymbol{\omega}=(\vartheta, \varphi) \in \operatorname{supp} \mu_{t_{0}}$ has representation (5.7). Moreover, it follows from Lemma 6.3 that $\vartheta \in W^{1,2}(\Omega) \mu_{t_{0}}$ - almost everywhere. Hence $\boldsymbol{\omega}$ meets all requirements of Theorem $4.2 \mu_{t_{0}}$ - almost everywhere. In view of relation (4.9) in this theorem the inequality

$$
\begin{aligned}
\Pi_{0}\left(\vartheta, \vartheta^{*}\left(t_{0}\right)\right)+ & \Pi_{1}\left(H^{*}\left(t_{0}\right), \varphi\right) \geq \\
& \quad \liminf _{t_{1} \nearrow t_{0}} \frac{1}{t_{0}-t_{1}}\left\{\mathbf{M}\left(v\left(t_{0}\right), w\left(t_{0}\right)\right)-\mathbf{M}\left(v\left(t_{1}\right), w\left(t_{1}\right)\right)\right\}
\end{aligned}
$$

holds true for all $t_{1} \in \mathcal{D}$. From this and relation (6.14) in Lemma 6.2 we obtain

$$
\Pi_{0}\left(\vartheta, \vartheta^{*}\left(t_{0}\right)\right)+\Pi_{1}\left(H^{*}\left(t_{0}\right), \varphi\right) \geq-\mathbf{M}^{\prime}\left(t_{0}\right)
$$

for $\mu_{t_{0}}$ - a.e. $(\vartheta, \varphi)$. Integrating both sides of this inequality with respect to the probability measure $\mu_{t_{0}}$ we obtain

$$
\int_{\Sigma_{\eta}}\left(\Pi_{0}\left(\vartheta, \vartheta^{*}\left(t_{0}\right)\right)+\Pi_{1}\left(H^{*}\left(t_{0}\right), \varphi\right)\right) d \mu_{t_{0}}(\boldsymbol{\omega}) \geq-\mathbf{M}^{\prime}\left(t_{0}\right) .
$$

Combining this result with (6.16) we arrive at desired inequality (6.24).

We are now in a position to prove that $\mu_{t_{0}}$ is the Dirac measure.

Lemma 6.5 Let $t_{0} \in \mathcal{D}$ be a Lebesgue point of $\mathcal{D}$. Then the measure $\mu_{t_{0}}$ is the Dirac measure concentrated at point $\boldsymbol{\omega}^{*}=\left(\vartheta^{*}\left(t_{0}\right), \varphi^{*}\left(t_{0}\right)\right)$. Moreover, the functions $\vartheta^{*}\left(t_{0}\right)$ and $\varphi^{*}\left(t_{0}\right)$ admit representations $(6.2)$ and $(6.3)$.

Proof First we prove the identities

$$
\begin{gathered}
\int_{\Sigma_{\eta}} \Pi_{0}\left(\vartheta, \vartheta^{*}\left(t_{0}\right)\right) d \mu_{t_{0}}=\Pi_{0}\left(\vartheta^{*}\left(t_{0}\right), \vartheta^{*}\left(t_{0}\right)\right), \\
\int_{\Sigma_{\eta}} \Pi_{1}\left(H^{*}\left(t_{0}\right), \varphi\right) d \mu_{t_{0}}=\Pi_{1}\left(H^{*}\left(t_{0}\right), \varphi^{*}\left(t_{0}\right)\right)=\int_{\Sigma_{\eta}} \Pi_{1}\left(H(\varphi), \varphi^{*}\left(t_{0}\right)\right) d \mu_{t_{0}}, \\
\int_{\Sigma_{\eta}} \Pi_{1}(H(\varphi), \varphi) d \mu_{t_{0}}=\Pi_{1}\left((H \varphi)^{*}\left(t_{0}\right), 1\right) .
\end{gathered}
$$


Here $(H \varphi)^{*}$ is a weak limit of the sequence $H\left(\varphi_{N}\right) \varphi_{N}$ in $L^{s}\left(0, T ; L^{q}(\Omega)\right)$. In order to prove (6.25) notice that the function $\Pi_{0}^{(n)}$ given by (6.9) admits the representation

$$
\Pi_{0}^{(n)}\left(\vartheta, \vartheta^{*}\left(t_{0}\right)\right)=\int_{\Omega} v^{(n)} \vartheta d x, \quad v^{(n)}=\sum_{1}^{n} \lambda_{k} c_{k} \zeta_{k} \in C(\Omega)
$$

where $c_{k}$ are the Fourier coefficients of $\vartheta^{*}\left(t_{0}\right)$ in the basis $\zeta_{k}$. Since the sequence $\vartheta_{N}$ converges to $\vartheta^{*}$ weakly in $L^{r}\left(0, T ; L^{p}(\Omega)\right)$ with $r>1$ and $p>2$ we have

$$
\lim _{N \rightarrow \infty} \int_{\left[t_{1}, t_{0}\right] \cap \mathcal{F}_{\eta}} \Pi_{0}^{(n)}\left(\vartheta_{N}(t), \vartheta^{*}\left(t_{0}\right)\right) d t=\int_{\left[t_{1}, t_{0}\right] \cap \mathcal{F}_{\eta}} \int_{\Omega} v^{(n)} \vartheta^{*}(t) d x d t .
$$

In view of Theorem 4.4 the function $\vartheta^{*}$ belongs to the space $C\left(\mathcal{F}_{\eta} ; L^{p}(\Omega)\right)$. Since $t_{0}$ is a Lebesgue point of $\mathcal{D} \subset \mathcal{F}_{\eta}$, we conclude from this that

$$
\begin{aligned}
\lim _{t_{1} \rightarrow t_{0}} \frac{1}{t_{0}-t_{1}} \int_{\left[t_{1}, t_{0}\right] \cap \mathcal{F}_{\eta}} \int_{\Omega} v^{(n)} \vartheta^{*}(t) d x d t & = \\
\int_{\Omega} v^{(n)} \vartheta^{*}\left(t_{0}\right) d x & =\Pi_{0}^{(n)}\left(\vartheta^{*}\left(t_{0}\right), \vartheta^{*}\left(t_{0}\right)\right) .
\end{aligned}
$$

On the other hand, relation (6.28) implies that $\Pi_{0}^{(n)}\left(\vartheta, \vartheta^{*}\left(t_{0}\right)\right)$ is a continuous function of $\vartheta$ on $X$ Hence we can apply relation (5.3) in Theorem 5.1 to obtain

$$
\begin{aligned}
\lim _{N \rightarrow \infty} \int_{\left.\left[t_{1}, t_{0}\right] \cap \mathcal{F}_{\eta}\right]} \Pi_{0}^{(n)}\left(\vartheta_{N}(t), \vartheta^{*}\left(t_{0}\right)\right) d t= \\
\int_{\left[t_{1}, t_{0}\right] \cap \mathcal{F}_{\eta} \times \Sigma_{\eta}} \Pi_{0}^{(n)}\left(\vartheta, \vartheta^{*}\left(t_{0}\right)\right) d \nu(\boldsymbol{\omega}) .
\end{aligned}
$$

Next, relation (5.5) in Theorem 5.1 yields

$$
\begin{gathered}
\lim _{t_{1} \rightarrow t_{0}} \frac{1}{t_{0}-t_{1}} \int_{\left[t_{1}, t_{0}\right] \cap \mathcal{F}_{\eta} \times \Sigma_{\eta}} \Pi_{0}^{(n)}\left(\vartheta, \vartheta^{*}\left(t_{0}\right)\right) d \nu(\boldsymbol{\omega})= \\
\int_{\Sigma_{\eta}} \Pi_{0}^{(n)}\left(\vartheta, \vartheta^{*}\left(t_{0}\right)\right) d \mu_{t_{0}}(\boldsymbol{\omega}) .
\end{gathered}
$$

Combining (6.28)-(6.31) we finally arrive at

$$
\int_{\Sigma_{\eta}} \Pi_{0}^{(n)}\left(\vartheta, \vartheta^{*}\left(t_{0}\right)\right) d \mu_{t_{0}}(\boldsymbol{\omega})=\Pi_{0}^{(n)}\left(\vartheta^{*}\left(t_{0}\right), \vartheta^{*}\left(t_{0}\right)\right) .
$$

Recall that $\Pi_{0}$ is integrable with respect to measure $\mu_{t_{0}}$ and $\Pi_{0}^{(n)} \nearrow \Pi_{0}$ in $W^{1,2}(\Omega)$. Notice that $\vartheta \in W^{1,2}(\Omega)$ for $\mu_{t_{0}}$ almost every point $(\vartheta, \varphi)$. Letting $n \rightarrow \infty$ in (6.32) and applying the Fatou theorem we arrive at (6.25). Now 
our task is to prove the first equality in (6.26). Recall that $\varphi_{N} \rightarrow \varphi^{*}$ weakly in $L^{s}\left(0, T ; L^{q}(\Omega)\right)$ and $H^{*} \in L^{\infty}(\Omega)$. Thus we get

$$
\lim _{N \rightarrow \infty} \int_{\left[t_{1}, t_{0}\right] \cap \mathcal{F}_{\eta}} \Pi_{1}\left(H^{*}\left(t_{0}\right), \varphi_{N}(t)\right) d t=\int_{\left[t_{1}, t_{0}\right] \cap \mathcal{F}_{\eta}} \int_{\Omega} H^{*}\left(t_{0}\right) \varphi^{*}(t) d x d t .
$$

On the other hand, relation (6.28) implies that $\Pi_{1}\left(H^{*}\left(t_{0}\right), \varphi\right)$ is a continuous function of $\varphi$ on $X$. Hence we can apply relations (5.3) in Theorem 5.1 to obtain

$$
\lim _{N \rightarrow \infty} \int_{\left[t_{1}, t_{0}\right] \cap \mathcal{T}_{\eta}} \Pi_{1}\left(H^{*}\left(t_{0}\right), \varphi_{N}(t)\right) d t=\int_{\left(\left[t_{1}, t_{0}\right] \cap \mathcal{T}_{\eta}\right) \times \Sigma_{\eta}} \Pi_{1}\left(H^{*}\left(t_{0}\right), \varphi\right) d \nu(\boldsymbol{\omega}) .
$$

Thus we get

$$
\begin{gathered}
\int_{\left[t_{1}, t_{0}\right] \cap \mathcal{F}_{\eta}} \int_{\Omega} H^{*}\left(t_{0}\right) \varphi^{*}(t) d x d t= \\
\int_{\left(\left[t_{1}, t_{0}\right] \cap \mathcal{T}_{\eta}\right) \times \Sigma_{\eta}} \Pi_{1}\left(H^{*}\left(t_{0}\right), \varphi\right) d \nu(\boldsymbol{\omega}) .
\end{gathered}
$$

Since the mapping $\mathcal{F}_{\eta} \ni t \rightarrow \varphi^{*}(t) \in L^{q}(\Omega)$ is continuous and $t_{0}$ is a Lebesgue point of $\mathcal{F}_{\eta}$, we have

$$
\begin{gathered}
\lim _{t_{1} \rightarrow t_{0}} \frac{1}{t_{0}-t_{1}} \int_{\left[t_{1}, t_{0}\right] \cap \mathcal{T}_{\eta}} \int_{\Omega} H^{*}\left(t_{0}\right) \varphi^{*}(t) d x d t= \\
\int_{\Omega} H^{*}\left(t_{0}\right) \varphi^{*}\left(t_{0}\right) d x=\Pi_{1}\left(H^{*}\left(t_{0}\right), \varphi^{*}\left(t_{0}\right)\right) .
\end{gathered}
$$

Next, relation (5.5) in Theorem 5.1 implies

$$
\begin{gathered}
\lim _{t_{1} \rightarrow t_{0}} \frac{1}{t_{0}-t_{1}} \int_{\left(\left[t_{1}, t_{0}\right] \cap \mathcal{T}_{\eta}\right) \times \Sigma_{\eta}} \Pi_{1}\left(H^{*}\left(t_{0}\right), \varphi\right) d \nu(\boldsymbol{\omega}) \\
=\int_{\Sigma_{\eta}} \Pi_{1}\left(H^{*}\left(t_{0}\right), \varphi\right) d \mu_{t_{0}}(\boldsymbol{\omega}) .
\end{gathered}
$$

Combining (6.33)-(6.35) we arrive at the first equality in (6.26). Arguing as before we obtain

$$
\lim _{N \rightarrow \infty} \int_{\left[t_{1}, t_{0}\right] \cap \mathcal{F}_{\eta}} \Pi_{1}\left(H\left(\varphi_{N}\right), \varphi^{*}\left(t_{0}\right) d t=\int_{\left[t_{1}, t_{0}\right] \cap \mathcal{F}_{\eta}} \int_{\Omega} H^{*}\left(t_{0}\right) \varphi^{*}(t) d x d t\right.
$$

and

$$
\lim _{N \rightarrow \infty} \int_{\left[t_{1}, t_{0}\right] \cap \mathcal{T}_{\eta}} \Pi_{1}\left(H\left(\varphi_{N}\right), \varphi^{*}\left(t_{0}\right)\right) d t=\int_{\left(\left[t_{1}, t_{0}\right] \cap \mathcal{T}_{\eta}\right) \times \Sigma_{\eta}} \Pi_{1}\left(H(\varphi), \varphi^{*}\left(t_{0}\right)\right) d \nu(\boldsymbol{\omega}),
$$

which leads to

$$
\begin{gathered}
\int_{\left[t_{1}, t_{0}\right] \cap \mathcal{F}_{\eta}} \int_{\Omega} H^{*}\left(t_{0}\right) \varphi^{*}(t) d x d t= \\
\int_{\left(\left[t_{1}, t_{0}\right] \cap \mathcal{T}_{\eta}\right) \times \Sigma_{\eta}} \Pi_{1}\left(H(\varphi), \varphi^{*}\left(t_{0}\right)\right) d \nu(\boldsymbol{\omega}) .
\end{gathered}
$$


Relation (5.5) in Theorem 5.1 implies

$$
\begin{gathered}
\lim _{t_{1} \rightarrow t_{0}} \frac{1}{t_{0}-t_{1}} \int_{\left(\left[t_{1}, t_{0}\right] \cap \mathcal{T}_{\eta}\right) \times \Sigma_{\eta}} \Pi_{1}\left(H(\varphi), \varphi^{*}\left(t_{0}\right)\right) d \nu(\boldsymbol{\omega})= \\
\int_{\Sigma_{\eta}} \Pi_{1}\left(H(\varphi), \varphi^{*}\left(t_{0}\right)\right) d \mu_{t_{0}}(\boldsymbol{\omega}) .
\end{gathered}
$$

Combining (6.34), (6.36), and (6.37) we obtain the second equality in (6.26). The proof of inequality (6.27) is similar.

We are now in a position to show that $\mu_{t_{0}}$ is the Dirac measure. Using identities (6.25)-(6.26) we rewrite the right hand side of inequality (6.24) in the form

$$
\begin{gathered}
\int_{\Sigma_{\eta}}\left(\Pi_{0}\left(\vartheta, \vartheta^{*}\left(t_{0}\right)\right)+\Pi_{1}\left(H^{*}\left(t_{0}\right), \varphi\right)\right) d \mu_{t_{0}}=-\int_{\Sigma_{\eta}} \Pi_{0}\left(\vartheta^{*}\left(t_{0}\right), \vartheta^{*}\left(t_{0}\right)\right) d \mu_{t_{0}}+ \\
2 \int_{\Sigma_{\eta}} \Pi_{0}\left(\vartheta, \vartheta^{*}\left(t_{0}\right)\right) d \mu_{t_{0}}+\Pi_{1}\left(H^{*}\left(t_{0}\right), \varphi^{*}\left(t_{0}\right)\right) .
\end{gathered}
$$

Next, using identity (6.27) and recalling that $\mu_{t_{0}}$ is a probability measure we rewrite the left hand side of (6.24) in the form

$$
\int_{\Sigma_{\eta}} \Pi(\vartheta, \varphi) d \mu_{t_{0}}=\int_{\Sigma_{\eta}} \Pi_{0}(\vartheta, \vartheta) d \mu_{t_{0}}+\Pi_{1}\left((H \varphi)^{*}\left(t_{0}\right), 1\right) .
$$

Substituting these results in (6.24) we arrive at the important inequality

$$
\begin{gathered}
\int_{\Sigma_{\eta}} \Pi_{0}\left(\vartheta-\vartheta^{*}\left(t_{0}\right), \vartheta-\vartheta^{*}\left(t_{0}\right)\right) d \mu_{t_{0}}+ \\
\left.\Pi_{1}\left((H \varphi)^{*}\left(t_{0}\right), 1\right)-\Pi_{1}\left(H^{*}\left(t_{0}\right), \varphi^{*}\left(t_{0}\right)\right)\right) \leq 0 .
\end{gathered}
$$

Since $\Pi_{0} \geq 0$, it follows that

$$
\begin{aligned}
& \left.\Pi_{1}\left((H \varphi)^{*}\left(t_{0}\right), 1\right)-\Pi_{1}\left(H^{*}\left(t_{0}\right), \varphi^{*} t_{0}\right)\right) \equiv \\
& \left.\quad \int_{\Omega}\left((H \varphi)^{*}\left(x, t_{0}\right)-H^{*}\left(x, t_{0}\right), \varphi^{*}\left(x, t_{0}\right)\right)\right) d x \leq 0 \text { for all } t_{0} \in \mathcal{D} .
\end{aligned}
$$

Let us prove that $H^{*}\left(t_{0}\right)=H\left(\varphi^{*}\left(t_{0}\right)\right)$. The proof of this fact is based on the representation of the weak limits in terms of the Young measure. Notice that $\varphi_{N} \rightarrow \varphi^{*}$ star weakly in $L^{\infty}\left(\mathcal{F}_{\eta} ; L^{q}(\Omega)\right), q>1$. Since the function $H$ is bounded and continuous, it follows from the fundamental theorem on the Young measures that there is a measurable family of probability measures $\sigma_{x, t}$ such that

$$
\varphi^{*}=\int_{\mathbb{R}} \lambda d \sigma_{x, t}(\lambda), H^{*}=\int_{\mathbb{R}} H(\lambda) d \sigma_{x, t}(\lambda),(H \varphi)^{*}=\int_{\mathbb{R}} H(\lambda) \lambda d \sigma_{x, t}(\lambda) .
$$


almost everywhere in $\Omega \times \mathcal{F}_{\eta}$. Since $\sigma_{x, t}$ is a probability measure, we have

$$
\begin{gathered}
(H \varphi)^{*}\left(x, t_{0}\right)-H^{*}\left(x, t_{0}\right), \varphi^{*}\left(x, t_{0}\right)=\int_{\mathbb{R}}(H(\lambda) \lambda-\bar{\lambda} \bar{H}) d \sigma_{x, t}(\lambda)= \\
\int_{\mathbb{R}}(H(\lambda)-\bar{H})(\lambda-\bar{\lambda}) d \sigma_{x, t}=\int_{\mathbb{R}} H(\lambda)(\lambda-\bar{\lambda}) d \sigma_{x, t}=\int_{\mathbb{R}}(H(\lambda)-H(\bar{\lambda}))(\lambda-\bar{\lambda}) d \sigma_{x, t}
\end{gathered}
$$

a.e. in $\Omega \times \mathcal{F}_{\eta}$. It follows from this and (6.39) that

$$
\int_{\mathbb{R}}(H(\lambda)-H(\bar{\lambda}))(\lambda-\bar{\lambda}) d \sigma_{x, t} \leq 0
$$

almost everywhere in $\Omega \times \mathcal{F}_{\eta}$. Since the function $H$ is strictly monotone, it is possible if and only if $\sigma_{x, t}$ is the Dirac measure for a.e. $(x, t) \in \Omega \times \mathcal{F}_{\eta}$. From this and general theory of the Young measures we conclude that $\varphi_{N} \rightarrow \varphi$ in measure in $\Omega \times \mathcal{F}_{\eta}$. It follows that $H^{*}\left(t_{0}\right)=H\left(\varphi^{*}\left(t_{0}\right)\right.$ for a.e. $t \in \mathcal{F}_{\eta}$. Thus we get

$\Pi_{1}\left(H^{*}\left(t_{0}\right), \varphi\right)=\Pi_{1}\left(H\left(\varphi^{*}\left(t_{0}\right)\right), \varphi\right), \quad \Pi_{1}\left(H^{*}\left(t_{0}\right), \varphi^{*}\left(t_{0}\right)\right)=\Pi_{1}\left(H\left(\varphi^{*}\left(t_{0}\right)\right), \varphi^{*}\left(t_{0}\right)\right)$.

From this and (6.26) we obtain

$$
\begin{aligned}
\int_{\Sigma_{\eta}} \Pi_{1}\left(H^{*}\left(t_{0}\right), \varphi\right) d \mu_{t_{0}}=\int_{\Sigma_{\eta}}\left\{\Pi_{1}\left(H\left(\varphi^{*}\left(t_{0}\right)\right), \varphi\right)+\Pi_{1}\left(H(\varphi), \varphi^{*}\left(t_{0}\right)\right)\right\} d \mu_{t_{0}} \\
\left.-\int_{\Sigma_{\eta}} \Pi_{1}\left(H\left(\varphi^{*}\left(t_{0}\right)\right), \varphi^{*}\left(t_{0}\right)\right)\right\} d \mu_{t_{0}} . \quad(6.40)
\end{aligned}
$$

On the other hand, equality (6.25) implies

$$
\begin{aligned}
\int_{\Sigma_{\eta}} \Pi_{0}\left(\vartheta, \vartheta^{*}\left(t_{0}\right)\right) d \mu_{t_{0}}=2 \int_{\Sigma_{\eta}} \Pi_{0}\left(\vartheta, \vartheta^{*}\left(t_{0}\right)\right) d \mu_{t_{0}} \\
\quad-\int_{\Sigma_{\eta}} \Pi_{0}\left(\vartheta^{*}\left(t_{0}\right), \vartheta^{*}\left(t_{0}\right)\right) d \mu_{t_{0}}
\end{aligned}
$$

Substituting (6.40) and (6.41) into inequality (6.24) we may rewrite this inequality in the equivalent form

$$
\begin{gathered}
\int_{\Sigma_{\eta}} \Pi_{0}\left(\vartheta-\vartheta^{*}\left(t_{0}\right), \vartheta-\vartheta^{*}\left(t_{0}\right)\right) d \mu_{t_{0}}+ \\
\int_{\Sigma_{\eta}} \Pi_{1}\left(\left(H(\varphi)-H\left(\varphi^{*}\left(t_{0}\right)\right)\right)\left(\varphi-\varphi^{*}\left(t_{0}\right)\right) d \mu_{t_{0}} \leq 0 .\right.
\end{gathered}
$$

Notice that the integrands in the left hand side of this inequality are nonnegative and equal zero if and only if $\vartheta=\vartheta^{*}\left(t_{0}\right)$ and $\varphi=\varphi^{*}\left(t_{0}\right)$. Hence $\mu_{t_{0}}$ is the Dirac measure concentrated in $\left(\vartheta^{*}\left(t_{0}\right), \varphi^{*}\left(t_{0}\right)\right)$. This completes the proof of Lemma 6.1. In remains to note that in view of Theorem 5.2 representations (5.7) and (5.8) hold for every element of supp $\mu_{t_{0}}$. Hence they hold for $\left(\vartheta^{*}\left(t_{0}\right), \varphi^{*}\left(t_{0}\right)\right)$ which yields $(6.2)$ and $(6.3)$. 
Finally we prove the strong convergence of the sequences $\vartheta_{N}$ and $\varphi_{N}$.

Lemma 6.6 Let exponents $(r, p)$ and $(s, q)$ satisfy condition (2.20). Then we have

$$
\vartheta_{N} \rightarrow \vartheta^{*} \text { in } L^{r}\left(0, T ; L^{p}(\Omega)\right), \quad \varphi_{N} \rightarrow \varphi^{*} \text { in } L^{s}\left(0, T ; L^{q}(\Omega)\right) .
$$

Proof Choose $\gamma>1$ satisfying the inequality $\gamma<\min \{r, p, s, q\}$ and notice that the mapping

$$
F:(\vartheta, \varphi) \rightarrow \int_{\Omega}\left(|\vartheta|^{\gamma}+|\varphi|^{\gamma}\right) d x
$$

is continuous on $X$. Lemma 6.5 implies

$$
\int_{\Sigma_{\eta}} F(\vartheta, \varphi) d \mu_{t_{0}}=F\left(\vartheta^{*}\left(t_{0}\right), \varphi^{*}\left(t_{0}\right)\right)=\int_{\Omega}\left(\left|\vartheta^{*}\left(x, t_{0}\right)\right|^{\gamma}+\left|\varphi^{*}\left(x, t_{0}\right)\right|^{\gamma}\right) d x .
$$

for a.e. $t_{0} \in \mathcal{F}_{\eta}$. Applying Theorem 5.1 we obtain that

$$
\begin{aligned}
& \lim _{N \rightarrow \infty} \int_{\mathcal{F}_{\eta}} \int_{\Omega}\left(\left|\vartheta_{N}\right|^{\gamma}+|\varphi|^{\gamma}\right) d x d t=\lim _{N \rightarrow \infty} \int_{\mathcal{F}_{\eta}} F\left(\vartheta_{N}(t), \varphi_{N}(t)\right) d x d t= \\
& \int_{\mathcal{F}_{\eta}}\left\{\int_{\Sigma_{\eta}} F(\vartheta, \varphi) d \mu_{t}\right\} d t=\int_{\mathcal{F}_{\eta}} \int_{\Omega}\left(\left|\vartheta^{*}(x, t)\right|^{\gamma}+\left|\varphi^{*}(t)\right|^{\gamma}\right) d x d t
\end{aligned}
$$

Recall that the sequence $\left(\vartheta_{N}, \varphi_{N}\right)$ converges to $\left(\vartheta^{*}, \varphi^{*}\right)$ weakly in $L^{\gamma}(\Omega \times$ $(0, T))$. Since $F$ is strictly convex, it follows from this and (6.44) that $\left(\vartheta_{N}, \varphi_{N}\right) \rightarrow$ $\left(\vartheta^{*}, \varphi^{*}\right)$ in $L^{\gamma}\left(\Omega \times \mathcal{F}_{\eta}\right)$. In particular, the sequence $\left(\vartheta_{N}, \varphi_{N}\right)$ converges in measure in $\Omega \times \mathcal{F}_{\eta}$. Letting $\eta \rightarrow 0$ we conclude that this sequence converges to $\left(\vartheta^{*}, \varphi^{*}\right)$ in measure in $\Omega \times(0, T)$. It follows from (4.4) that the sequence $\vartheta_{N}$ is bounded in $L^{r}\left(0, T ; L^{p}(\Omega)\right)$ and the sequence $\varphi_{n}$ is bounded in $L^{s}\left(0, T ; L^{p}(\Omega)\right)$ for all exponents $(r, p)$ and $(s, q)$ satisfying the inequalities (2.20). Since these sequences converge in measure and the set of admissible exponents $(r, p)$ and $(s, q)$ is open, we conclude that $\vartheta_{N} \rightarrow \vartheta^{*}$ in $L^{r}\left(0, T ; L^{p}(\Omega)\right)$ and $\varphi_{N} \rightarrow \varphi^{*}$ strongly in $L^{s}\left(0, T ; L^{q}(\Omega)\right)$.

It remains to note that Theorem 5.2 is a straightforward consequence of Lemmas 6.5 and 6.6 .

\section{Proof of Theorem 1.1}

In this section we complete the proof of the main Theorem 1.1. Let us consider the sequence of the approximate solutions $\vartheta_{n}, v_{n}$ and $w_{N}$ defined by Theorem 2.1. We begin with the observation that Theorems 3.1 and 6.1 imply the relations

$$
\begin{gathered}
w_{N}^{ \pm 1} \rightarrow w^{ \pm 1} \text { in } C\left(0, T ; L^{\alpha}(\Omega)\right) \\
\left(v_{N}, \vartheta_{N}\right) \rightarrow\left(v, \vartheta^{*}\right) \text { in } L^{r}\left(0, T ; L^{p}(\Omega)\right), \\
\varphi_{N} \equiv \varphi\left(D^{2} \mathbf{u}_{N}, D \mathbf{u}_{N}, \vartheta_{N}, w_{N}\right) \rightarrow \varphi^{*} \text { in } L^{s}\left(0, T ; L^{q}(\Omega)\right),
\end{gathered}
$$


which hold true for all $\alpha \in[1, \infty)$ and for all $(p, r),(s, q)$ satisfying $(2.20)$. The limits satisfy the conditions

$$
\begin{gathered}
w^{ \pm 1} \in L^{\infty}\left(0, T ; L^{\infty}(\Omega)\right) \cap L^{\infty}\left(0, T ; W^{1,2}(\Omega)\right), \partial_{t} w \in L^{2}\left(0, T ; W^{1,2}(\Omega)\right), \\
v, \vartheta^{*} \in L^{2}\left(0, T ; W^{1,2}(\Omega)\right) \cap L^{\infty}\left(0, T ; L^{2}(\Omega)\right) .
\end{gathered}
$$

Moreover, Theorem 6.1 implies that for almost every $t \in(0, T)$, there is a function $\mathbf{u}(t) \in \mathcal{W}^{2,2}(\Omega)+\mathbf{h}$ such that $\|\mathbf{u}(t)\|_{W^{2,6}(\Omega)} \leq c_{0}$ and

$$
\begin{gathered}
\vartheta^{*}(t)=v(t)+W\left(w(t)^{-1} D \mathbf{u}(t)\right), \quad \varphi^{*}(t)=\varphi\left(D^{2} \mathbf{u}(t), D \mathbf{u}(t), \vartheta^{*}(t), w(t)\right), \\
\mathcal{H}(\mathbf{u}(t), v(t), w(t))=\mathbf{M}(v(t), w(t)) .
\end{gathered}
$$

Let us prove that the functions $v, w, \mathbf{u}, \vartheta^{*}$ meet all requirements of Definition 1.1 and serve as a weak solution to problem (1.20). It suffices to prove that these functions satisfy equations $(1.20 \mathrm{c}),(1.20 \mathrm{~g})$ and integral identities (1.25) and (1.26). Notice that equation (2.7) in the definition od the approximate solution yields

$$
\partial_{t} w_{N}=-H\left(\varphi\left(D^{2} \mathbf{u}_{N}, D \mathbf{u}_{N}, \vartheta_{n}, w_{N}\right)\right) w_{N}, \quad 0<t \leq T, w_{N}(0)=w_{0} .
$$

Letting $N \rightarrow \infty$ and using relations (7.1)-(7.2) we conclude that $w$ satisfies equation and initial condition $(1.20 \mathrm{c})$ and $(1.20 \mathrm{~g})$. Next, integral identity (4.22) implies that $w$ and $\vartheta^{*}$ satisfy integral identity (1.25) with $\vartheta$ replaced by $\vartheta^{*}$. In view of the definition (1.31) of the marginal functional the vector field $\mathbf{u}(t)$ is a minimizer of the functional $\mathcal{H}(\mathbf{u}, v(t), w(t))$. Hence, the equality

$$
\lim _{\lambda \rightarrow 0} \lambda^{-1}(\mathcal{H}(\mathbf{u}+\boldsymbol{\xi}, v(t), w(t))-\mathcal{H}(\mathbf{u}, v(t), w(t)))=0
$$

holds for every function $\boldsymbol{\xi}$ vanishing at $\partial \Omega$. Recall that the functional $\mathcal{H}$ is defined by

$$
\mathcal{H}(\mathbf{u}, v(t), w(t)))=\mathcal{E}(\mathbf{u}, v(t), w(t)))+\int_{\Omega} \mathbf{u} \cdot \mathbf{f} d x,
$$

where the integral functional $\mathcal{E}$ is given by (1.29). Substituting the expressions for $\mathcal{H}$ into (7.5) we obtain the integral identity

$$
\begin{aligned}
\int_{\Omega}\left(\frac{\varepsilon}{w(t)} \Delta \mathbf{u}(t) \cdot \Delta \boldsymbol{\xi}+\right. \\
\left.\quad w(t)^{2}(1+\Theta(D \mathbf{u}(t), v(t))) W^{\prime}\left(w^{-1}(t) D \mathbf{u}(t)\right): D \boldsymbol{\xi}+f \cdot \boldsymbol{\xi}\right) d x=0 .
\end{aligned}
$$

Noting that $\vartheta^{*}=\Theta(D \mathbf{u}(t), v(t))$ we conclude that $\mathbf{u}, w$ and $\vartheta^{*}$ satisfy integral identity (1.26). Next, notice that in view of (7.4) the displacement field $\mathbf{u}(t)$ satisfies the first selection principle given by Definition 1.3.

It remains to prove that $\vartheta^{*}(t)$ and $\varphi^{*}=\varphi\left(D^{2} \mathbf{u}(t), D \mathbf{u}(t), \vartheta^{*}(t), w(t)\right.$ satisfy the second selection principle formulated in Definition 1.5. To this end choose a minimizer $\tilde{\mathbf{u}} \in \mathcal{W}^{2,2}+\mathbf{h}$ of the functional $\mathcal{H}\left(\cdot, \mathbf{v}\left(t_{0}\right), w\left(t_{0}\right)\right)$ and set

$$
\tilde{\vartheta}=v\left(t_{0}\right)+W\left(w\left(t_{0}\right)^{-1} D \tilde{\mathbf{u}}\right), \quad \tilde{\varphi}=\varphi\left(D^{2} \tilde{\mathbf{u}}, D \tilde{\mathbf{u}}, \tilde{\vartheta}, w(t)\right) .
$$


It follows that $(\tilde{\vartheta}, \tilde{\varphi}) \in \mathcal{P}\left(v\left(t_{0}\right), w\left(t_{0}\right)\right)$, where the set $\mathcal{P}(v, w)$ is given by Definition 1.4. Notice that the function $\tilde{\mathbf{u}}$ meets all requirements of Theorem 4.2 with $\mathbf{u}$ replaced by $\tilde{\mathbf{u}}$. Recall that the function $\mathbf{M}\left(v\left(t_{0}\right), w\left(t_{0}\right)\right)$ is differentiable at a.e. point $t_{0} \in(0, T)$. From this and relation (4.9) in Theorem 4.2 we obtain

$$
\Pi_{0}\left(\tilde{\vartheta}, \vartheta^{*}\left(t_{0}\right)\right)+\Pi_{1}\left(\tilde{\varphi}, H^{*}\left(t_{0}\right)\right) \geq-\mathbf{M}^{\prime}\left(v\left(t_{0}\right), w\left(t_{0}\right)\right) \text { for a.e. } t_{0} \in(0, T) .
$$

Obviously we have $\vartheta_{N} \rightarrow \vartheta^{*}$ weakly in $L^{2}\left(0, T ; W^{1,2}(\Omega)\right)$. Let us consider the sequence of the functions $\bar{\vartheta}_{N}$ given by (2.9)-(2.10). In view of Theorem 2.1 they are bounded in $L^{2}\left(t_{1}, t_{0} ; W^{1,2}(\Omega)\right)$ and $\vartheta_{N}-\bar{\vartheta}_{N} \rightarrow 0$ in $L^{2}\left(t_{1}, t_{0} ; L^{2}(\Omega)\right)$. It follows that $\bar{\vartheta}_{N} \rightarrow \vartheta^{*}$ weakly in $L^{2}\left(t_{1}, t_{0} ; W^{1,2}(\Omega)\right.$. Thus we get

$$
\liminf _{N \rightarrow \infty}\left\{\frac{1}{2} \int_{t_{1}+\tau}^{t_{0}} \Pi_{0}\left(\vartheta_{N}, \vartheta_{N}\right) d s+\frac{1}{2} \int_{t_{1}+\tau}^{t_{0}-\tau} \Pi_{0}\left(\bar{\vartheta}_{N}, \bar{\vartheta}_{N}\right) d s\right\} \geq \int_{t_{1}}^{t_{0}} \Pi_{0}\left(\vartheta^{*}, \vartheta^{*}\right) .
$$

It obviously follows from (7.1) that

$$
\lim _{N \rightarrow \infty} \int_{t_{1}+\tau}^{t_{0}} \Pi_{1}\left(H\left(\vartheta_{N}\right), \vartheta_{N}\right) d s=\int_{t_{1}}^{t_{0}} \Pi_{1}\left(H\left(\vartheta^{*}\right), \vartheta^{*}\right) d s .
$$

Letting $N \rightarrow \infty$ in relation (4.2) in Theorem 4.1, and using (7.7) and (7.8) we get the inequality

$$
\frac{1}{t_{0}-t_{1}}\left(\mathbf{M}\left(v\left(t_{0}\right), w\left(t_{0}\right)\right)-\mathbf{M}\left(v\left(t_{1}\right), w\left(t_{1}\right)\right)\right)+\frac{1}{t_{0}-t_{1}} \int_{t_{1}}^{t_{0}} \Pi\left(\vartheta^{*}, \varphi^{*}\right) d t \leq 0 .
$$

Letting $t_{1} \rightarrow t_{0}$ we arrive at the estimate

$$
\Pi\left(\vartheta^{*}\left(t_{0}\right), \varphi^{*}\left(t_{0}\right)\right) \leq-\mathbf{M}^{\prime}\left(v\left(t_{0}\right), w\left(t_{0}\right)\right) \text { for a.e. } t_{0} \in(0, T) .
$$

Combining this estimate with $(7.6)$ we conclude that $\vartheta^{*}$ and $\varphi^{*}$ satisfy inequality (1.35). Hence $\vartheta^{*}$ and $\varphi^{*}$ satisfy the second selection principle. This completes the proof of Theorem 1.1.

\section{A Proof of Theorem 2.1}

Step1. First we prove the solvability of problem (2.4)-(2.6). Our task is to show that there exist functions $\mathbf{u}_{n}, \vartheta_{n}, w_{n}$, and $v_{n}, 1 \leq n \leq N$, satisfying (2.5)-(2.6). We proceed by the induction principle. Assume that

$$
v_{k} \in L^{2}(\Omega), \quad \vartheta_{k} \in W^{1,2}(\Omega), \quad w_{k}^{ \pm 1} \in L^{\infty}(\Omega), \quad \mathbf{u}_{k}-\mathbf{h} \in \mathcal{W}^{2,2}
$$

are defined for all $k \leq n-1$. We aim to show that there are $\left(\mathbf{u}_{n}, v_{n}, \vartheta_{n}, w_{n}^{ \pm 1}\right)$ satisfying (2.5)-(2.6). We begin with the observation that the functional $W^{1,2}(\Omega) \ni$ $\vartheta \rightarrow \mathbf{S}_{n}(\mathbf{u}, \vartheta)$ is strictly concave, continuous, and bounded from above for every $\mathbf{u} \in W^{2,2}(\Omega)$. Hence there exists a unique $\vartheta_{n}(\mathbf{u}) \in W^{1,2}(\Omega)$ such that

$$
\mathbf{S}_{n}\left(\mathbf{u}, \vartheta_{n}(\mathbf{u})\right)=\max _{\vartheta \in W^{1,2}(\Omega)} \mathbf{S}_{n}(\mathbf{u}, \vartheta)
$$

The following lemma gives the explicit expression for the left hand side of this relation. 
Lemma A.1 Let $\mathbf{u}-\mathbf{h} \in \mathcal{W}^{2,2}$ and $\vartheta_{n}=\vartheta_{n}(\mathbf{u})$. Then we have

$$
\mathbf{S}_{n}\left(\mathbf{u}, \vartheta_{n}\right)=\mathbf{E}\left(\mathbf{u}, \vartheta_{n}, w_{n-1}\right)+\frac{\tau}{2} \Pi_{0}\left(\vartheta_{n}, \vartheta_{n}\right)-\int_{\Omega} \mathbf{f} \cdot \mathbf{u} d x
$$

Proof Calculation of the variation of $\mathbf{S}_{n}$ at the point $\vartheta_{n}=\vartheta_{n}(\mathbf{u})$ leads to the linear elliptic boundary boundary value problem for $\vartheta_{n}$

$$
\begin{array}{r}
-\tau \Delta \vartheta_{n}+w_{n-1}^{3} \vartheta_{n}=w_{n-2}^{3} v_{n-1}+w_{n-1}^{3} W\left(w_{n-1}^{-1} D \mathbf{u}\right) \text { in } \Omega, \\
\partial_{n} \vartheta_{n}+\vartheta_{n}=0 \text { on } \partial \Omega .
\end{array}
$$

Since $w_{n-1}, w_{n-2}$ are uniformly bounded and $W\left(w_{n-1}^{-1} D \mathbf{u}\right), v_{n-1} \in L^{2}(\Omega)$, it follows from the general theory of elliptic boundary value problems, see [9], that problem (A.3) has a unique solution $\vartheta_{n}=\vartheta_{n}(\mathbf{u}) \in W^{2,2}(\Omega)$ and the mapping

$$
W^{2,2}(\Omega) \ni \mathbf{u} \rightarrow \vartheta_{n}(\mathbf{u}) \in W^{2,2}(\Omega)
$$

is continuous. Multiplying both sides of (A.3) by $\vartheta_{n}$ and integrating the result by parts we arrive at the identity

$$
\int_{\Omega} w_{n-2}^{3} v_{n-1} \vartheta_{n} d x=\int_{\Omega} w_{n-1}^{3} V\left(D \mathbf{u}, \vartheta_{n}, w_{n-1}\right) \vartheta_{n} d x+\tau \Pi_{0}\left(\vartheta_{n}, \vartheta_{n}\right) .
$$

Combining this result with the expression (2.1) for $\mathbf{S}_{n}$ and noting that

$$
\boldsymbol{\Psi}_{g}\left(\mathbf{u}, \vartheta_{n}, w_{n-1}\right)=\mathbf{E}\left(\mathbf{u}, \vartheta_{n}, w_{n-1}\right)+\int_{\Omega} w_{n-1}^{3} V\left(D \mathbf{u}, \vartheta_{n}, w_{n-1}\right) \vartheta_{n} d x
$$

we arrive at (A.2).

It follows from (A.2) and the continuity of the mapping (A.4) that the functional

$$
W^{2,2}(\Omega) \ni \mathbf{u} \rightarrow \mathbf{S}_{n}\left(\mathbf{u}, \vartheta_{n}(\mathbf{u})\right) \in \mathbb{R}
$$

is the sum of the strictly convex and weakly continuous parts. It is obviously bounded from below. Hence there is $\mathbf{u}_{n} \in \mathcal{W}^{2,2}+\mathbf{h}$ such that

$$
\mathbf{S}_{n}\left(\mathbf{u}_{n}, \vartheta_{n}\left(\mathbf{u}_{n}\right)\right)=\min _{\mathbf{u}-\mathbf{h} \in \mathcal{W}^{2,2}} \mathbf{S}_{n}\left(\mathbf{u}, \vartheta_{n}(\mathbf{u})\right)=\min _{\mathbf{u}-\mathbf{h} \in \mathcal{W}^{2,2}} \max _{\vartheta \in W^{1,2}} \mathbf{S}_{n}(\mathbf{u}, \vartheta) .
$$

Thus we prove the existence of functions $\left(\mathbf{u}_{n}, \vartheta_{n}\right) \in W^{2,2}(\Omega) \times W^{2,2}(\Omega)$ satisfying (2.5).

Now our task is to find $w_{n}$. We begin with the observation that $\mathbf{u}_{n}$ and $\vartheta_{n}$ are independent of $t$. From this and equality (1.20e) we conclude that for a.e. $x \in \Omega$, the functions $\varphi\left(D^{2} \mathbf{u}_{n}, D \mathbf{u}_{n}, \vartheta_{n}, w\right)$ and $H\left(\varphi\left(D^{2} \mathbf{u}_{n}, D \mathbf{u}_{n}, \vartheta_{n}, w\right)\right)$ are continuously differentiable with respect to $w$ on the interval $(0, \infty)$. Since the functions $w_{n-1}^{ \pm 1}$ are uniformly bounded, the Cauchy problem

$$
\partial_{t} w=-H\left(\varphi\left(\vartheta_{n}, D^{2} \mathbf{u}_{n}, D \mathbf{u}_{n}, w\right)\right), \quad w((n-1) \tau)=w_{n-1},
$$

has a unique solution defined in a neighborhood of $(n-1) \tau$. Moreover, since the function $H$ is uniformly bounded and $t \in(0, T)$, this solution admits the estimates

$$
\begin{aligned}
\left(\left\|w_{n-1}\right\|_{L^{\infty}(\Omega)}+\left\|w_{n-1}^{-1}\right\|_{L^{\infty}(\Omega)}\right)^{-1} e^{-C T} \leq & w(t) \leq \\
& \left(\left\|w_{n-1}\right\|_{L^{\infty}(\Omega)}+\left\|w_{n-1}^{-1}\right\|_{L^{\infty}(\Omega)}\right) e^{C t},
\end{aligned}
$$

where $C=\sup |H|$. Hence it can be extended to the interval $((n-1) \tau, n \tau]$. Denote this extension as $w_{n}(x, t)$. By construction, it satisfies (2.7). Let us show that $w_{n}$ 
is uniformly bounded from below and above. To this end, notice that the function $w_{N}(x, t)$ given by $(2.2)$ is defined on the interval $(0, T]$ and satisfies the equation and initial condition

$$
\partial_{t} w_{N}=-H\left(\varphi\left(D^{2} \mathbf{u}_{N}, D \mathbf{u}_{n}, \vartheta_{N}, w_{N}\right)\right) w_{N} \text { for } t \in(0, n \tau), w(0)=w_{0} .
$$

Since $|H| \leq C$, the function $w_{N}$ satisfies the inequalities

$$
\begin{array}{r}
0<c^{-1} \leq w_{N}(x, t) \leq c<\infty \text { for } 0 \leq t \leq n \tau, \\
\left|\partial_{t} w_{N}(x, t)\right| \leq c<\infty \text { for } 0 \leq t \leq n \tau .
\end{array}
$$

Thus we find $\mathbf{u}_{n}, \vartheta_{n}$ and $w_{n}$ satisfying (2.5)-(2.7). It remains to note that $v_{n}$ is given by the formula (2.6). Since in view of growth condition (1.21) we have

$$
\left|W\left(w_{n}^{-1} D \mathbf{u}_{n}\right)\right| \leq c(1+|\mathbf{D} u|)^{3} \text { and } \mathbf{u}-\mathbf{h} \in \mathcal{W}^{2,2},
$$

it follows from the embedding theorem that $W\left(w_{n}^{-1} D \mathbf{u}_{n}\right) \in L^{2}(\Omega)$ and hence $v_{n} \in L^{2}(\Omega)$. Applying the induction principle we conclude that problem (2.4)-(2.6) has a solution

$$
\begin{gathered}
\mathbf{u}_{N} \in L^{\infty}\left(0, T ; W^{2,2}(\Omega)\right), \quad \vartheta_{N} \in L^{\infty}\left(0, T ; W^{2,2}(\Omega)\right), \\
w_{N}^{ \pm 1} \in L^{\infty}\left(0, T ; L^{\infty}(\Omega)\right), \quad v_{N} \in L^{\infty}\left(0, T ; L^{2}(\Omega)\right) .
\end{gathered}
$$

Moreover, in view of (A.6) the growth factor $w_{N}$ satisfies the inequalities

$$
0<c^{-1} \leq w_{N}(x, t) \leq c<\infty, \quad\left|\partial_{t} w_{N}(x, t)\right| \leq c<\infty \text { for } 0 \leq t \leq T .
$$

Step 2. Our next task is to derive energy estimate (2.11). First we derive the auxiliary inequality (A.18) which leads to the desired energy estimate. The proof of this inequality is purely algebraic. We begin with the observation that

$$
\begin{aligned}
& \mathbf{S}_{n}\left(\mathbf{u}_{n}, \vartheta_{n}\right)=\min _{\mathbf{u}-\mathbf{h} \in \mathcal{W}^{2,2}} \max _{\vartheta \in W^{1,2}} \mathbf{S}_{n}(\mathbf{u}, \vartheta) \\
& \leq \max _{\vartheta \in W^{1,2}} \mathbf{S}_{n}\left(\mathbf{u}_{n-1}, \vartheta\right)=\mathbf{S}_{n}\left(\mathbf{u}_{n-1}, \bar{\vartheta}_{n-1}\right) .
\end{aligned}
$$

where $\bar{\vartheta}_{n-1}$ is a solution to the variational problem

$$
\mathbf{S}_{n}\left(\mathbf{u}_{n-1}, \bar{\vartheta}_{n-1}\right)=\max _{\vartheta \in W^{1,2}(\Omega)} \mathbf{S}_{n}\left(\mathbf{u}_{n-1}, \vartheta\right)
$$

Next, notice that problem (A.10) is a particular case of variational problem (A.1) with $\mathbf{u}=\mathbf{u}_{n-1}$. Arguing as before we conclude that variational problem (A.10) has a unique solution $\bar{\vartheta}_{n-1} \in W^{2,2}(\Omega)$. Expression (2.1) for $\mathbf{S}_{n}$ implies

$$
\begin{array}{r}
\mathbf{S}_{n}\left(\mathbf{u}_{n-1}, \bar{\vartheta}_{n-1}\right)=\boldsymbol{\Psi}_{g}\left(\mathbf{u}_{n-1}, \bar{\vartheta}_{n-1}, w_{n-1}\right)-\frac{\tau}{2} \Pi_{0}\left(\bar{\vartheta}_{n-1}, \bar{\vartheta}_{n-1}\right)+ \\
\int_{\Omega}\left(w_{n-2}^{3} v_{n-1} \bar{\vartheta}_{n-1}-\mathbf{f} \cdot \mathbf{u}_{n-1}\right) d x
\end{array}
$$

Notice that the integrand $\Psi_{g}$ is a concave function of $\vartheta$, which leads to the inequality

$$
\begin{aligned}
& \Psi_{g}\left(D^{2} \mathbf{u}_{n-1}, D \mathbf{u}_{n-1}, \bar{\vartheta}_{n-1},\right.\left.w_{n-1}\right) \leq \Psi_{g}\left(D^{2} \mathbf{u}_{n-1}, D \mathbf{u}_{n-1}, \vartheta_{n-1}, w_{n-1}\right)+ \\
& \partial_{\vartheta} \Psi_{g}\left(D^{2} \mathbf{u}_{n-1}, D \mathbf{u}_{n-1}, \vartheta_{n-1}, w_{n-1}\right)\left(\bar{\vartheta}_{n-1}-\vartheta_{n-1}\right) .
\end{aligned}
$$


Noting that

$$
\begin{array}{r}
\partial_{\vartheta} \Psi_{g}\left(D^{2} \mathbf{u}_{n-1}, D \mathbf{u}_{n-1}, \vartheta_{n-1}, w_{n-1}\right)=-V\left(D \mathbf{u}_{n-1}, \vartheta_{n-1}, w_{n-1}\right), \\
E\left(D^{2} \mathbf{u}_{n-1}, D \mathbf{u}_{n-1}, \vartheta_{n-1}, w_{n-1}\right)=\Psi_{g}\left(D^{2} \mathbf{u}_{n-1}, D \mathbf{u}_{n-1}, \vartheta_{n-1}, w_{n-1}\right)+ \\
w_{n-1}^{3} V\left(D \mathbf{u}_{n-1}, \vartheta_{n-1}, w_{n-1}\right) \vartheta_{n-1},
\end{array}
$$

and recalling representation (1.29) for the internal energy $\mathbf{E}$, we obtain

$$
\begin{aligned}
& \boldsymbol{\Psi}_{g}\left(\mathbf{u}_{n-1}, \bar{\vartheta}_{n-1}, w_{n-1}\right) \leq \mathbf{E}\left(\mathbf{u}_{n-1}, \vartheta_{n-1}, w_{n-1}\right)- \\
& \qquad \int_{\Omega} w_{n-1}^{3} V\left(D \mathbf{u}_{n-1}, \vartheta_{n-1}, w_{n-1}\right) \bar{\vartheta}_{n-1} d x .
\end{aligned}
$$

Substituting this estimate into (A.11) and noting that $v_{n-1}=V\left(D \mathbf{u}_{n-1}, \vartheta_{n-1}, w_{n-2}\right)$ we obtain

$$
\begin{array}{r}
\mathbf{S}_{n}\left(\mathbf{u}_{n-1}, \bar{\vartheta}_{n-1}\right) \leq \mathbf{E}\left(\mathbf{u}_{n-1}, \vartheta_{n-1}, w_{n-1}\right)-\frac{\tau}{2} \Pi_{0}\left(\bar{\vartheta}_{n-1}, \bar{\vartheta}_{n-1}\right) \\
-\int_{\Omega} \mathbf{f} \cdot \mathbf{u}_{n-1} d x+\int_{\Omega} Q_{n-2, n-1} \bar{\vartheta}_{n-1} d x,
\end{array}
$$

where

$$
Q_{n-2, n-1}=w_{n-2}^{3} V\left(D \mathbf{u}_{n-1}, \vartheta_{n-1}, w_{n-2}\right)-w_{n-1}^{3} V\left(D \mathbf{u}_{n-1}, \vartheta_{n-1}, w_{n-1}\right) .
$$

On the other hand, representation (A.2) implies

$$
\mathbf{S}_{n}\left(\mathbf{u}_{n}, \vartheta_{n}\right)=\mathbf{E}\left(\mathbf{u}_{n}, \vartheta_{n}, w_{n-1}\right)-\int_{\Omega} \mathbf{f} \cdot \mathbf{u}_{n} d x+\frac{\tau}{2} \Pi_{0}\left(\vartheta_{n}, \vartheta_{n}\right)
$$

Substituting this identity and inequality (A.12) into inequality (A.9) we get

$$
\begin{array}{r}
\mathbf{E}\left(\mathbf{u}_{n}, \vartheta_{n}, w_{n}\right)-\mathbf{E}\left(\mathbf{u}_{n-1}, \vartheta_{n-1},, w_{n-1}\right)+\frac{\tau}{2}\left(\Pi_{0}\left(\vartheta_{n}, \vartheta_{n}\right)+\Pi_{0}\left(\bar{\vartheta}_{n-1}, \bar{\vartheta}_{n-1}\right)\right) \leq \\
\mathbf{E}\left(\mathbf{u}_{n}, \vartheta_{n}, w_{n}\right)-\mathbf{E}\left(\mathbf{u}_{n}, \vartheta_{n}, w_{n-1}\right)+\int_{\Omega} \mathbf{f} \cdot\left(\mathbf{u}_{n}-\mathbf{u}_{n-1}\right) d x+\int_{\Omega} Q_{n-2, n-1} \bar{\vartheta}_{n-1} d x .
\end{array}
$$

On the other hand, the identity

$$
\mathbf{E}(\mathbf{u}, \vartheta, w)=\boldsymbol{\Psi}_{g}(\mathbf{u}, \vartheta, w)+\int_{\Omega} w^{3} \vartheta V(D \mathbf{u}, \vartheta, w) d x
$$

implies the representation

$$
\begin{array}{r}
\mathbf{E}\left(\mathbf{u}_{n}, \vartheta_{n}, w_{n}\right)-\mathbf{E}\left(\mathbf{u}_{n}, \vartheta_{n}, w_{n-1}\right)+\int_{\Omega} Q_{n-2, n-1} \bar{\vartheta}_{n-1} d x= \\
\mathbf{A}_{n}-\mathbf{A}_{n-1}+\mathbf{B}_{n}+\mathbf{C}_{n},
\end{array}
$$

where

$$
\begin{array}{r}
\mathbf{A}_{n}=-\int_{\Omega} Q_{n-1, n} \vartheta_{n} d x, \quad \mathbf{B}_{n}=\int_{\Omega} Q_{n-2, n-1}\left(\bar{\vartheta}_{n-1}-\vartheta_{n-1}\right) d x, \\
\mathbf{C}_{n}=\boldsymbol{\Psi}_{g}\left(\mathbf{u}_{n}, \vartheta_{n}, w_{n}\right)-\boldsymbol{\Psi}_{g}\left(\mathbf{u}_{n}, \vartheta_{n}, w_{n-1}\right) .
\end{array}
$$

Substituting (A.15) into (A.14) we finally obtain

$$
\begin{array}{r}
\mathbf{E}\left(\mathbf{u}_{n}, \vartheta_{n}, w_{n}\right)-\mathbf{E}\left(\mathbf{u}_{n-1}, \vartheta_{n-1}, w_{n-1}\right)+\frac{\tau}{2}\left(\Pi_{0}\left(\vartheta_{n}, \vartheta_{n}\right)+\Pi_{0}\left(\bar{\vartheta}_{n-1}, \bar{\vartheta}_{n-1}\right)\right) \\
\leq \int_{\Omega} \mathbf{f} \cdot\left(\mathbf{u}_{n}-\mathbf{u}_{n-1}\right) d x+\mathbf{A}_{n}-\mathbf{A}_{n-1}+\mathbf{B}_{n}+\mathbf{C}_{n} .
\end{array}
$$


Summing both sides with respect to $n$ and noting that

$\tau \sum_{n=l+1}^{m} \Pi_{0}\left(\vartheta_{n}, \vartheta_{n}\right)=\int_{\tau l}^{\tau m} \Pi_{0}\left(\vartheta_{N}, \vartheta_{N}\right) d t, \quad \tau \sum_{n=l}^{m-1} \Pi_{0}\left(\bar{\vartheta}_{n}, \bar{\vartheta}_{n}\right) \geq \int_{\tau l}^{\tau(m-1)} \Pi_{0}\left(\bar{\vartheta}_{N}, \bar{\vartheta}_{N}\right) d t$

we conclude that for all integers $0 \leq l<m \leq N$,

$$
\begin{array}{r}
\left\{\mathbf{E}\left(\mathbf{u}_{m}, \vartheta_{m}, w_{m}\right)-\int_{\Omega} \mathbf{f} \cdot \mathbf{u}_{m} d x\right\}-\left\{\mathbf{E}\left(\mathbf{u}_{l}, \vartheta_{l}, w_{l}\right)-\int_{\Omega} \mathbf{f} \cdot \mathbf{u}_{l} d x\right\}+ \\
\frac{1}{2} \int_{\tau l}^{\tau m} \Pi_{0}\left(\vartheta_{N}, \vartheta_{N}\right) d t+\frac{1}{2} \int_{\tau l}^{\tau(m-1)} \Pi_{0}\left(\bar{\vartheta}_{N}, \bar{\vartheta}_{N}\right) d t \leq \mathbf{A}_{m}-\mathbf{A}_{l}+\sum_{n=l+1}^{m}\left(\mathbf{B}_{n}+\mathbf{C}_{n}\right) .
\end{array}
$$

Setting $l=0$ in (A.17) we finally arrive at the estimate

$$
\begin{array}{r}
\mathbf{E}\left(\mathbf{u}_{m}, \vartheta_{m}, w_{m}\right)-\int_{\Omega} \mathbf{f} \cdot \mathbf{u}_{m} d x+\frac{1}{2} \int_{0}^{\tau m} \Pi_{0}\left(\vartheta_{N}, \vartheta_{N}\right) d t \leq \\
\left|\mathbf{A}_{m}\right|+\sum_{n=1}^{m}\left(\left|\mathbf{B}_{n}\right|+\mathbf{C}_{n}\right)+c .
\end{array}
$$

Step 3. Now our task is to estimate the right hand side of (A.18). Introduce the quantities

$$
\mathbf{I}_{n}=\int_{\Omega}\left(\left|\Delta \mathbf{u}_{n}\right|^{2}+W\left(w_{n-1}^{-1} D \mathbf{u}_{n}\right)+\vartheta_{n}^{2}\right) d x, \quad n \geq 0 .
$$

It obviously follows from formula (1.29) for $\mathbf{E}$ and the inequality $W \geq 0$ that

$$
c^{-1} \mathbf{I}_{n} \leq \mathbf{E}\left(\mathbf{u}_{n}, \vartheta_{n}, w_{m}\right) \leq c \mathbf{I}_{n}
$$

Moreover, since the embedding $W^{2,2}(\Omega) \hookrightarrow W^{1,6}(\Omega)$ is bounded and $\mathbf{u}=\mathbf{h}$ on $\partial \Omega$, we have

$$
\int_{\Omega}\left|D \mathbf{u}_{n}\right|^{6} d x \leq c\left(1+\int_{\Omega}\left|\Delta \mathbf{u}_{n}\right|^{2} d x\right)^{3} \leq c\left(\mathbf{I}_{n}^{3}+1\right), \quad \int_{\Omega}\left|\vartheta_{n}\right|^{2} d x \leq c \mathbf{I}_{n} .
$$

Here the constant $c$ is independent of $n$. Our first task is to estimate $\mathbf{A}_{m}$ in terms of $\mathbf{I}_{m}$. Expression (A.16) for $\mathbf{A}_{m}$ implies

$$
\left|\mathbf{A}_{m}\right| \leq \int_{\Omega}\left|\vartheta_{m}\right|\left|Q_{m-1, m} w_{m}^{3}\right| \cdot d x
$$

It follows from (A.13) and (1.27) that

$$
\left|Q_{m-1, m}\right| \leq\left|\vartheta_{m}\right|\left|w_{m}^{3}-w_{m-1}^{3}\right|+\left|w_{m}^{3} W\left(w_{m}^{-1} D \mathbf{u}_{m}\right)-w_{m-1}^{3} W\left(w_{m-1}^{-1} D \mathbf{u}_{m}\right)\right| .
$$

In view of (A.8) we have

$$
\left|w_{n}\right|^{ \pm 1}+\left|w_{n-1}\right|^{ \pm 1} \leq c, \quad\left|w_{n}-w_{n-1}\right| \leq c \tau \text { for all } 1 \leq n \leq N .
$$

From this and (1.21) we obtain the estimate

$$
\left|Q_{m-1, m}\right| \leq c \tau\left|\vartheta_{m}\right|+c \tau\left(\left|D \mathbf{u}_{m}\right|^{3}+1\right)
$$

which along with the Cauchy inequality implies

$$
\left|\mathbf{A}_{m}\right| \leq(c \tau+\delta) \int_{\Omega}\left|\vartheta_{m}\right|^{2} d x+c(\delta) \tau^{2} \int_{\Omega}\left(\left|D \mathbf{u}_{m}\right|^{6}+1\right) d x .
$$


Here $\delta$ is an arbitrary positive number. Combining this and (A.21) we arrive at the desired estimate for $\mathbf{A}_{m}$ :

$$
\left|\mathbf{A}_{m}\right| \leq(c \tau+\delta) \mathbf{I}_{m}+c(\delta) \tau^{2}\left(1+\mathbf{I}_{m}^{3}\right) .
$$

The derivation of the estimate for $\mathbf{B}_{n}$ is based on the following lemma.

Lemma A.2 The estimate

$$
\tau \Pi_{0}\left(\vartheta_{n-1}-\bar{\vartheta}_{n-1}, \vartheta_{n-1}-\bar{\vartheta}_{n-1}\right)+\int_{\Omega}\left|\vartheta_{n-1}-\bar{\vartheta}_{n-1}\right|^{2} d x \leq c \tau\left(1+\mathbf{I}_{n}\right)^{3}
$$

holds true for all $1 \leq n \leq N$. Here the constant $c$ is independent of $n$ and $N$.

Proof The variation of the functional $S_{n}\left(\mathbf{u}_{n-1}, \vartheta\right)$ at the extremal point $\vartheta=\bar{\vartheta}_{n-1}$ leads to the following equations for $\bar{\vartheta}_{n-1}$ :

$$
\begin{array}{r}
-\tau \Delta \bar{\vartheta}_{n-1}+w_{n-1}^{3} \bar{\vartheta}_{n-1}=w_{n-2}^{3} v_{n-1}+w_{n-1}^{3} W\left(w_{n-1}^{-1} D \mathbf{u}_{n-1}\right) \text { in } \Omega \\
\partial_{n} \bar{\vartheta}_{n-1}+\bar{\vartheta}_{n-1}=0 .
\end{array}
$$

In view of (A.3) we have

$$
\begin{array}{r}
-\tau \Delta \vartheta_{n-1}+w_{n-2}^{3} \vartheta_{n-1}=w_{n-3}^{3} v_{n-2}+w_{n-2}^{3} W\left(w_{n-2}^{-1} D \mathbf{u}_{n-1}\right) \text { in } \Omega \\
\partial_{n} \vartheta_{n-1}+\vartheta_{n-1}=0 \text { on } \partial \Omega .
\end{array}
$$

Notice that equation (A.28) can be written in the equivalent form

$$
w_{n-2}^{3} v_{n-1}-w_{n-3}^{3} v_{n-2}=\tau \Delta \vartheta_{n-1} .
$$

It follows from (A.27)-(A.29) that the function $\zeta=\bar{\vartheta}_{n-1}-\vartheta_{n-1}$ satisfies the equations and boundary conditions.

$$
-\tau \Delta \zeta+w_{n-1}^{3} \zeta=Q_{n-2, n-1}+\tau \Delta \vartheta_{n-1}, \quad \partial_{n} \zeta+\zeta=0 \text { on } \partial \Omega,
$$

where $Q_{n-2, n-1}$ is given by (A.13). Recall that $\vartheta_{n-1} \in W^{2,2}(\Omega)$. Multiplying both sides of this equation by $\zeta$, integrating by parts and using the Cauchy inequality we obtain

$$
\tau \Pi_{0}(\zeta, \zeta)+c \int_{\Omega}|\zeta|^{2} d x \leq \tau \Pi_{0}\left(\zeta, \vartheta_{n-1}\right)+\delta \int_{\Omega}|\zeta|^{2} d x+\delta^{-1} \int_{\Omega}\left|Q_{m-2, m-1}\right|^{2} d x
$$

where the positive constant $c$ is independent of $n$ and $\mathbf{f}$. Notice that

$$
\Pi_{0}\left(\zeta, \vartheta_{n-1}\right) \leq \delta \Pi_{0}(\zeta, \zeta)+\delta^{-1} \Pi_{0}\left(\vartheta_{n-1}, \vartheta_{n-1}\right) .
$$

Choosing $\delta \leq \min \{c / 4,1 / 2\}$ we arrive at the estimate

$$
\tau \Pi_{0}(\zeta, \zeta)+\int_{\Omega}|\zeta|^{2} d x \leq c \int_{\Omega}\left|Q_{m-2, m-1}\right|^{2} d x+c \tau \Pi_{0}\left(\vartheta_{n-1}, \vartheta_{n-1}\right) .
$$

Next, inequality (A.24) and estimate (A.21) imply that

$$
\int_{\Omega}\left|Q_{m-2, m-1}\right|^{2} d x \leq c \tau^{2}\left(1+\mathbf{I}_{n-1}^{3}\right) .
$$

Substituting this estimate into (A.32) we obtain desired estimate (A.26). 
Next, formula (A.16) for the quantity $\mathbf{B}_{n}$ along with the Cauchy inequality yields the estimate

$$
\begin{gathered}
\left|\mathbf{B}_{n}\right| \leq \delta^{-1} \int_{\Omega}\left|Q_{n-2, n-1}\right|^{2} d x+\delta \int_{\Omega}\left(\vartheta_{n-1}-\bar{\vartheta}_{n-1}\right)^{2} d x \leq \\
c \tau^{2} \delta^{-1}\left(1+\mathbf{I}_{n-1}^{3}\right)+c \delta \tau \Pi_{0}\left(\vartheta_{n-1}, \vartheta_{n-1}\right)
\end{gathered}
$$

where $\delta$ is an arbitrary positive number. Summing both sides with respect to $n$ we arrive at the desired inequality

$$
\sum_{n=1}^{m}\left|\mathbf{B}_{n}\right| \leq c \tau^{2} \delta^{-1} \sum_{n=1}^{m}\left(1+\mathbf{I}_{n-1}\right)^{3}+c \delta \int_{0}^{\tau m} \Pi_{0}\left(\vartheta_{N}, \vartheta_{N}\right) d t
$$

Our next task is to estimate $\mathbf{C}_{n}$. To this end notice that

$$
\begin{aligned}
\mathbf{C}_{n} \equiv \boldsymbol{\Psi}_{g}\left(\mathbf{u}_{n}, \vartheta_{n}, w_{n}\right)- & \boldsymbol{\Psi}_{g}\left(\mathbf{u}_{n}, \vartheta_{n}, w_{n-1}\right)= \\
& \int_{0}^{1} \int_{\Omega} \frac{\partial \Psi_{g}}{\partial w}\left(D^{2} \mathbf{u}_{n}, D \mathbf{u}_{n}, \vartheta_{n}, \eta(x, s)\right)\left(w_{n}-w_{n-1}\right) d x d s,
\end{aligned}
$$

where

$$
\eta(x, s)=s w_{n}+(1-s) w_{n-1}, \quad s \in[0,1] .
$$

Relations (1.13a) and (1.20e) imply the identity

$$
\frac{\partial \Psi_{g}}{\partial w}\left(\vartheta_{n}, D^{2} \mathbf{u}_{n}, D \mathbf{u}_{n}, \eta(x, s)\right)=\frac{1}{\eta(x, s)} \varphi\left(D^{2} \mathbf{u}_{n}, D \mathbf{u}_{n}, \vartheta_{n}, \eta(s)\right) .
$$

In view of $(2.7)$ we have

$$
w_{n}-w_{n-1}=-\int_{\tau n-1}^{\tau n} H\left(\varphi\left(D^{2} \mathbf{u}_{n}, D \mathbf{u}_{n}, \vartheta_{n}, w_{N}(t)\right)\right) w_{N}(t) d t .
$$

Combining the obtaining result we arrive at the identity

$$
\begin{aligned}
& \quad \boldsymbol{\Psi}_{g}\left(\vartheta_{n}, \mathbf{u}_{n}, w_{n}\right)-\boldsymbol{\Psi}_{g}\left(\vartheta_{n}, \mathbf{u}_{n}, w_{n-1}\right)= \\
& -\int_{\tau(n-1)}^{\tau n} \int_{0}^{1} \int_{\Omega} \frac{1}{\eta(s)} \varphi\left(D^{2} \mathbf{u}_{n}, D \mathbf{u}_{n}, \vartheta_{n}, \eta(s)\right) H\left(\varphi\left(D^{2} \mathbf{u}_{n}, D \mathbf{u}_{n}, \vartheta_{n}, w_{N}(t)\right)\right) w_{N}(t) d x d s d t .
\end{aligned}
$$

Recalling the identity

$$
\varphi\left(D^{2} \mathbf{u}_{n}, D \mathbf{u}_{n}, \vartheta_{n}, w_{N}(t)\right)=\varphi_{N}(t) \text { for } t \in(\tau(n-1), \tau n]
$$

we can rewrite this relation in the form

$$
\boldsymbol{\Psi}_{g}\left(\vartheta_{n}, \mathbf{u}_{n}, w_{n}\right)-\boldsymbol{\Psi}_{g}\left(\vartheta_{n}, \mathbf{u}_{n}, w_{n_{1}}\right)=-\int_{\tau(n-1)}^{\tau n} \int_{\Omega} H\left(\varphi_{N}(\lambda)\right) \varphi_{N}(\lambda) d x d \lambda+\mathbf{R}_{n},
$$

where

$$
\begin{array}{r}
\mathbf{R}_{n}=\int_{\tau(n-1)}^{\tau n} \int_{0}^{1} \int_{\Omega} \mathbf{P}(x, s, t) H\left(\varphi_{N}(t)\right) w_{N}(t) d x d s d t, \\
\mathbf{P}=\frac{1}{w_{N}(t)} \varphi\left(D^{2} \mathbf{u}_{n}, D \mathbf{u}_{n}, \vartheta_{n}, w_{N}(t)\right)-\frac{1}{\eta(s)} \varphi\left(D^{2} \mathbf{u}_{n}, D \mathbf{u}_{n}, \vartheta_{n}, \eta(s)\right) .
\end{array}
$$


Let us estimate $\mathbf{R}_{n}$. We begin with the observation that $H$ is bounded and $w_{N}$ is uniformly bounded from below and above. Thus we get

$$
\mathbf{R}_{n} \leq \int_{\tau(n-1)}^{\tau n} \int_{0}^{1} \int_{\Omega}|\mathbf{P}(x, s, t)| d x d s d t .
$$

Next, we have for $s \in[0,1]$ and $t \in[\tau(n-1), \tau n]$,

$$
\mathbf{P}(x, s, t)=\left(w_{N}(t)-\eta(s)\right) \int_{0}^{1} \frac{\partial}{\partial \varsigma}\left(\frac{1}{\varsigma(\lambda)} \varphi\left(D^{2} \mathbf{u}_{n}, D \mathbf{u}_{n}, \vartheta_{n}, \varsigma(\lambda)\right)\right) d \lambda,
$$

where

$$
\varsigma=\lambda w_{N}(t)+(1-\lambda) \eta(s) \in\left[w_{N}(t), \eta(s)\right] .
$$

The rest of the proof is based on the following lemma.

Lemma A.3 Let $\varsigma: \Omega \rightarrow \mathbb{R}$ satisfies the inequalities $\left\|\varsigma^{ \pm 1}\right\|_{L^{\infty}(\Omega)} \leq c_{1}$. Then there is a constant $c\left(c_{1}\right)$ independent of $n$ such that

$$
\begin{gathered}
\int_{\Omega}\left(\left|\varphi\left(D^{2} \mathbf{u}_{n}, D \mathbf{u}_{n}, \vartheta_{n}, \varsigma\right)\right|+\left|\partial_{\varsigma} \varphi\left(D^{2} \mathbf{u}_{n}, D \mathbf{u}_{n}, \vartheta_{n}, \varsigma\right)\right|\right) d x \leq c\left(1+\mathbf{I}_{n}\right)^{3} \\
\int_{\Omega}\left|\partial_{\varsigma} E\left(D^{2} \mathbf{u}_{n}, D \mathbf{u}_{n}, \vartheta_{n}, \varsigma\right)\right| d x \leq c\left(1+\mathbf{I}_{n}\right)^{3}
\end{gathered}
$$

Proof Recall that

$$
\begin{array}{r}
\varphi\left(D^{2} \mathbf{u}_{n}, D \mathbf{u}_{n}, \vartheta_{n}, \varsigma\right)=-\frac{\varepsilon}{2 \varsigma}\left|\Delta \mathbf{u}_{n}\right|^{2}-\varsigma^{3} \frac{3 \vartheta_{n}^{2}}{2}+ \\
\varsigma^{3}\left(1+\vartheta_{n}\right)\left\{3 W\left(\varsigma^{-1} D \mathbf{u}_{n}\right)-\varsigma^{-1} W^{\prime}\left(\varsigma^{-1} D \mathbf{u}_{n}\right): D \mathbf{u}_{n}\right\} .
\end{array}
$$

The growth condition (1.21) implies

$$
\begin{array}{r}
\left|\varphi\left(D^{2} \mathbf{u}_{n}, D \mathbf{u}_{n}, \vartheta_{n}, \varsigma\right)\right|+\left|\partial_{\varsigma} \varphi\left(D^{2} \mathbf{u}_{n}, D \mathbf{u}_{n}, \vartheta_{n}, \varsigma\right)\right| \leq \\
\left(\left|\Delta \mathbf{u}_{n}\right|^{2}+\left|\vartheta_{n}\right|^{2}+\left(1+\left|\vartheta_{n}\right|\right)\left(1+\left|D \mathbf{u}_{n}\right|\right)^{3}\right) \leq c\left(1+\left|\Delta \mathbf{u}_{n}\right|^{2}+\left|\vartheta_{n}\right|^{2}\right)+c\left|D \mathbf{u}_{n}\right|^{6} .
\end{array}
$$

Integrating these inequalities over $\Omega$ and using estimate (A.21) we obtain desired estimate (A.39). Next, it follows from the expression (1.28) for the density of the internal energy $E$ that

$$
\left|\partial_{\varsigma} E\left(D^{2} \mathbf{u}_{n}, D \mathbf{u}_{n}, \vartheta_{n}, \varsigma\right)\right| \leq c\left(\left|\Delta \mathbf{u}_{n}\right|^{2}+\left|\vartheta_{n}\right|^{2}+\left|D \mathbf{u}_{n}\right|^{3}+1\right) .
$$

Arguing as before we arrive at (A.40) that

We are now in a position to derive the estimate for $\mathbf{C}_{n}$. It follows from (A.35)

$$
\left|\eta-w_{N}(t)\right| \leq\left|\eta-w_{n-1}\right|+\left|w_{N}(t)-w_{n-1}\right| \leq c \tau \text { for } t \in[(n-1) \tau n \tau],
$$

and

$$
0<w_{N}^{ \pm 1} \leq c, \quad 0<\eta^{ \pm 1} \leq c, \quad 0<\varsigma^{ \pm 1} \leq c .
$$

Applying inequality (A.39) in Lemma A.3 we obtain

$$
\begin{gathered}
\int_{\Omega}|\mathbf{P}(x, s, t)| d x \leq c \tau \int_{0}^{1} \int_{\Omega}\left|\frac{\partial}{\partial \varsigma}\left(\frac{1}{\varsigma(\lambda)} \varphi\left(D^{2} \mathbf{u}_{n}, D \mathbf{u}_{n}, \vartheta_{n}, \varsigma(\lambda)\right)\right)\right| d x d \lambda \leq \\
\leq c \tau \int_{0}^{1} \int_{\Omega}\left(\left|\varphi\left(D^{2} \mathbf{u}_{n}, D \mathbf{u}_{n}, \vartheta_{n}, \varsigma\right)\right|+\left|\partial_{\varsigma} \varphi\left(D^{2} \mathbf{u}_{n}, D \mathbf{u}_{n}, \vartheta_{n}, \varsigma\right)\right|\right) d x d \lambda \leq \\
c \tau \int_{0}^{1}\left(1+\mathbf{I}_{n}\right)^{3} d \lambda=c \tau\left(1+\mathbf{I}_{n}\right)^{3} .
\end{gathered}
$$


Combining this result with (A.38) we arrive at the inequality $\left|\mathbf{R}_{n}\right| \leq c \tau^{2}\left(1+\mathbf{I}_{n}\right)^{3}$. Substituting this inequality into (A.36) and recalling the expression (A.16) for $\mathbf{C}_{n}$ we finally obtain the desired estimate for $\mathbf{C}_{n}$

$$
\mathbf{C}_{n} \leq-\int_{\tau(n-1)}^{\tau n} \int_{\Omega} H\left(\varphi_{N}(t)\right) \varphi_{N}(t) d x d t+c \tau^{2}\left(1+\mathbf{I}_{n}\right)^{3} .
$$

Summing both the sides of this inequality with respect to $n$ and recalling expression (1.33) for the form $\Pi_{1}$ we arrive at the estimate

$$
\sum_{n=1}^{m} \mathbf{C}_{N} \leq-\int_{0}^{\tau m} \Pi_{1}\left(H\left(\varphi_{n}\right), \varphi_{n}\right)+c \tau^{2} \sum_{n=1}^{m}\left(1+\mathbf{I}_{n}\right)^{3} .
$$

Substituting (A.25), (A.34), and (A.42) into (A.18) we get the inequality

$$
\begin{array}{r}
\mathbf{E}\left(\mathbf{u}_{m}, \vartheta_{m}, w_{m}\right)-\int_{\Omega} \mathbf{f} \cdot \mathbf{u}_{m} d x+(1 / 2-c \delta) \int_{0}^{\tau m} \Pi_{0}\left(\vartheta_{N}, \vartheta_{N}\right)+ \\
\left.\int_{0}^{\tau m} \Pi_{1}\left(H\left(\varphi_{N}\right), \varphi_{N}\right) d t \leq c(\delta) \tau^{2} \sum_{n=1}^{m}\left(1+\mathbf{I}_{n}\right)^{3}\right)+c(\delta) .
\end{array}
$$

Noting that

$$
\int_{\Omega} \mathbf{f} \cdot \mathbf{u}_{m} d x \leq \delta \int_{\Omega}\left|\Delta \mathbf{u}_{m}\right|^{2} d x+c \delta^{-1} \leq \delta I_{m}+c \delta^{-1}
$$

we obtain

$$
\begin{gathered}
\mathbf{E}\left(\mathbf{u}_{m}, \vartheta_{m}, w_{m}\right)-\delta \mathbf{I}_{m}+(1 / 2-c \delta) \int_{0}^{\tau m} \Pi_{0}\left(\vartheta_{N}, \vartheta_{N}\right)+ \\
\left.\int_{0}^{\tau m} \Pi_{1}\left(H\left(\varphi_{N}\right), \varphi_{N}\right) d t \leq c(\delta) \tau^{2} \sum_{n=1}^{m}\left(1+\mathbf{I}_{n}\right)^{3}\right)+c(\delta) .
\end{gathered}
$$

Recalling estimate (A.20) and choosing $\delta$ sufficiently small we finally arrive at the inequality

$$
\left.\mathbf{I}_{m}+\int_{0}^{\tau m} \Pi\left(\vartheta_{N}, \varphi_{N}\right) d t \leq c \tau^{2} \sum_{n=1}^{m}\left(1+\mathbf{I}_{n}\right)^{3}\right)+c
$$

where the energy dissipation rate $\Pi$ is defined by (1.32). Let us estimate the right hand side of this equality. Set

$$
\mathbf{I}_{N}(t)=\mathbf{I}_{n} \quad \text { for }(n-1) \tau<t \leq n \tau, \quad \mathbf{J}_{N}(t)=\int_{0}^{t} \mathbf{I}_{N}^{3} d s .
$$

Since $\Pi$ is nonnegative, estimate (A.43) implies

$$
\mathbf{I}_{N}(t) \leq c+c \tau \int_{0}^{t} \mathbf{I}_{N}(s)^{3} d s, \text { for } 0<t \leq T .
$$

It follows that on the interval $(0, T)$ the function $\mathbf{J}_{N}$ satisfies the inequality

$$
\frac{d}{d t} \mathbf{J}_{N} \leq c\left(1+\tau \mathbf{J}_{N}\right)^{3} .
$$


Obviously $\mathbf{J}_{N}(t) \leq \sigma(t)$, where $\sigma$ is a solution to the Cauchy problem

$$
\frac{d}{d t} \sigma=c(1+\tau \sigma)^{3}, \quad \sigma(0)=0, \text { given by } \sigma(t)=\frac{1}{\tau}\left(\frac{1}{\sqrt{1-2 c \tau t}}-1\right) .
$$

For $\tau \leq 1 /(4 c T)$, the function $\sigma$ is defined and uniformly bounded on the interval $[0, T]$. This yields the estimate $\mathbf{J}_{N} \leq \sigma \leq c$. Combining this result with (A.44) we obtain the estimate

$$
\mathbf{I}_{n} \leq c \text { for } 0 \leq n \leq N,
$$

which along with (A.43) yields energy estimate (2.11). It remains to prove inequality (2.14). We begin with the observation that inequalities (A.45) and (A.25) implies the estimate

$$
\left|\mathbf{A}_{n}\right| \leq c(\delta+\tau)+c(\delta) \tau^{2} .
$$

Next, inequalities (A.45), (A.34), and (2.11) imply the estimate

$$
\sum_{n=1}^{N}\left|\mathbf{B}_{n}\right| \leq c \tau^{2} \delta^{-1} \sum_{l+1}^{N} 1+c \delta \int_{0}^{\tau N} \Pi_{0}\left(\vartheta_{N}, \vartheta_{N}\right) d t \leq c \tau \delta^{-1}+c \delta .
$$

In its turn, inequalities (A.42) and (A.34) imply

$$
\sum_{l+1}^{m} \mathbf{C}_{n} \leq-\int_{\tau l}^{\tau m} \int_{\Omega} H\left(\varphi_{N}(t)\right) \varphi_{N}(t) d x d t+c \tau .
$$

Substituting (A.46) -(A.48) into (A.17) and recalling the definition (1.32) of the energy dissipation rate $\Pi$ we obtain

$$
\begin{array}{r}
\left\{\mathbf{E}\left(\mathbf{u}_{m}, \vartheta_{m}, w_{m}\right)-\int_{\Omega} \mathbf{f} \cdot \mathbf{u}_{m} d x\right\}-\left\{\mathbf{E}\left(\mathbf{u}_{l}, \vartheta_{l}, w_{l}\right)-\int_{\Omega} \mathbf{f} \cdot \mathbf{u}_{l} d x\right\}+ \\
\frac{1}{2} \int_{\tau l}^{\tau m} \Pi_{0}\left(\vartheta_{N}, \vartheta_{N}\right) d t+\frac{1}{2} \int_{\tau l}^{\tau(m-1)} \Pi_{0}\left(\bar{\vartheta}_{N}, \bar{\vartheta}_{N}\right) d t+ \\
\int_{l \tau}^{m \tau} \Pi_{1}\left(H\left(\varphi_{N}\right), \varphi_{N}\right) d t \leq c \delta+c(\delta) \tau .
\end{array}
$$

Let us show that we can replace $w_{m}$ and $w_{l}$ in the left hand side of this inequality by $w_{m-1}$ and $w_{l-1}$. To this end notice that for every integer $n \in[1, N]$, we have

$$
\begin{gathered}
\mathbf{E}\left(\mathbf{u}_{m}, \vartheta_{m}, w_{m}\right)-\mathbf{E}\left(\mathbf{u}_{m}, \vartheta_{m}, w_{m-1}\right) \\
=\int_{0}^{1} \int_{\Omega} \partial_{\varsigma} E\left(D^{2} \mathbf{u}_{m}, D \mathbf{u}_{m}, \vartheta_{m}, \varsigma\right)\left(w_{m}-w_{m-1}\right) d x d s,
\end{gathered}
$$

where $\varsigma(s)=s w_{n}+(1-s) w_{n-1}$ satisfies the inequalities $\varsigma^{ \pm 1} \leq c$. From this, the inequality $\left|w_{n}-w_{n-1}\right| \leq c \tau$, and estimate (A.40) in Lemma A.3 we obtain

$$
\begin{gathered}
\left|\mathbf{E}\left(\mathbf{u}_{n}, \vartheta_{n}, w_{n}\right)-\mathbf{E}\left(\mathbf{u}_{n}, \vartheta_{n}, w_{n-1}\right)\right| \leq c \tau \int_{0}^{1} \int_{\Omega}\left|\partial_{\varsigma} E\left(D^{2} \mathbf{u}_{n}, D \mathbf{u}_{n}, \vartheta_{n}, \varsigma\right)\right| d x d s \leq \\
c \tau\left(1+\mathbf{I}_{n}\right)^{3} \leq c \tau .
\end{gathered}
$$

Combining this result with (A.49) and noting that $\mathbf{E}\left(\mathbf{u}_{m}, \vartheta_{m}, w_{m-1}\right)=\mathcal{E}\left(\mathbf{u}_{m}, v_{m}, w_{m-1}\right)$ we obtain

$$
\begin{array}{r}
\left\{\mathcal{E}\left(\mathbf{u}_{m}, v_{m}, w_{m-1}\right)-\int_{\Omega} \mathbf{f} \cdot \mathbf{u}_{m} d x\right\}-\left\{\mathcal{E}\left(\mathbf{u}_{l}, v_{l}, w_{l-1}\right)-\int_{\Omega} \mathbf{f} \cdot \mathbf{u}_{l} d x\right\}+ \\
\frac{1}{2} \int_{\tau l}^{\tau m} \Pi_{0}\left(\vartheta_{N}, \vartheta_{N}\right) d t+\frac{1}{2} \int_{\tau l}^{\tau(m-1)} \Pi_{0}\left(\bar{\vartheta}_{N}, \bar{\vartheta}_{N}\right) d t+ \\
\int_{l \tau}^{m \tau} \Pi_{1}\left(H\left(\varphi_{N}\right), \varphi_{N}\right) d t \leq c \delta+c(\delta) \tau
\end{array}
$$


It follows from the definition (2.15) of the functional $\mathcal{H}_{N}$ that

$$
\mathcal{H}_{N}(t)=\mathcal{E}\left(\mathbf{u}_{n}, v_{n}, w_{n-1}\right)-\int_{\Omega} \mathbf{f} \cdot \mathbf{u}_{n} d x \text { for } t \in((n-1) \tau, n \tau] .
$$

Thus we get

$$
\begin{array}{r}
\mathcal{H}_{N}(m \tau)-\mathcal{H}_{N}(l \tau)+\frac{1}{2} \int_{\tau l}^{\tau m} \Pi_{0}\left(\vartheta_{N}, \vartheta_{N}\right) d t \\
+\frac{1}{2} \int_{\tau l}^{\tau(m-1)} \Pi_{0}\left(\bar{\vartheta}_{N}, \bar{\vartheta}_{N}\right) d t+\int_{l \tau}^{m \tau} \Pi_{1}\left(H\left(\varphi_{N}\right), \varphi_{N}\right) d t \leq c \delta+c(\delta) \tau .
\end{array}
$$

Now fix $t_{1}<t_{0}$ from the interval $(0, T)$. For every $N$, choose $l$ and $m$ such that $t_{1} \in((l-1) \tau, l \tau]$ and $t_{0} \in((m-1) \tau, m \tau)$. We have

$$
\begin{gathered}
\frac{1}{2} \int_{\tau l}^{\tau m} \Pi_{0}\left(\vartheta_{N}, \vartheta_{N}\right) d t+\frac{1}{2} \int_{\tau l}^{\tau(m-1)} \Pi_{0}\left(\bar{\vartheta}_{N}, \bar{\vartheta}_{N}\right) d t+\int_{l \tau}^{m \tau} \Pi_{1}\left(H\left(\varphi_{N}\right), \varphi_{N}\right) d t \geq \\
\frac{1}{2} \int_{t_{1}+\tau}^{t_{0}} \Pi_{0}\left(\vartheta_{N}, \vartheta_{N}\right) d t+\frac{1}{2} \int_{t_{1}+\tau}^{t_{0}-\tau} \Pi_{0}\left(\bar{\vartheta}_{N}, \bar{\vartheta}_{N}\right) d t+\int_{t_{1}+\tau}^{t_{0}} \Pi_{1}\left(H\left(\varphi_{N}\right), \varphi_{N}\right) d t .
\end{gathered}
$$

Notice that $\boldsymbol{H}_{N}(t)$ is constant on every interval $((n-1) \tau, n \tau], 1 \leq n \leq N$. From this and (A.50) we conclude that

$$
\begin{array}{r}
\mathcal{H}_{N}\left(t_{0}\right)-\mathcal{H}_{N}\left(t_{1}\right)+\frac{1}{2} \int_{t_{1}+\tau}^{t_{0}} \Pi_{0}\left(\vartheta_{N}, \vartheta_{N}\right) d t+ \\
\frac{1}{2} \int_{t_{1}+\tau}^{t_{0}-\tau} \Pi_{0}\left(\bar{\vartheta}_{N}, \bar{\vartheta}_{N}\right) d t+\int_{t_{1}+\tau}^{t_{0}} \Pi_{1}\left(H\left(\varphi_{N}\right), \varphi_{N}\right) d t \leq c \delta+c(\delta) \tau .
\end{array}
$$

Letting $N \rightarrow \infty$ and then $\delta \rightarrow 0$ we obtain desired relation (2.14).

Acknowledgements P.I. Plotnikov was supported by Russian Science Foundation (project 15-11-20019) and by ANR-INTERLOCK3D for the stay at LEMTA and IECL (Nancy, France) in September-December, 2014, Jan Sokolowski was partially supported by ANR-12-BS01-0007 Optiform.

\section{References}

1. Alford P, Humphrey J., Taber L.: Growth and remodeling in a thick-walled artery model: effects of spatial variations in wall constituents. Biomech. and model. mech. 7, 245-262 (2008)

2. Ambrosio, L., Gigli, N., Savare G.: Gradient Flows in Metric Spaces and in Spaces of Probability Measures. Birkhäuser, Basel-Boston-Berlin (2005)

3. Ball J.: Convexity conditions ans existence theorems in nonlinear elasticity. Arch. Ration. Mech. Anal. 63, 337-403 (1976)

4. Ciarlet P.: Mathematical elasticity, 1: Three-dimensional Elasticity. Elsevier Science Publishers, Basel (1988)

5. Ciarletta, P., Ambrosi, D., Maugin, G. A.: Mass transport in morphogenetic processes: a second gradient theory for volumetric growth and material remodeling. J. Mech. Phys. Solids 60, 432-450 (2012)

6. Demirkoparan, H., Pence, T., Tsai, H.: Hyperelastic internal balance by multiplicative decomposition of the deformation gradient. Arch. Ration. Mech. Anal. 214, 923-970 (2014)

7. Epstein M., Maugin, G.: Thermomechanics of volumetric growth in uniform bodies. Int. J. Plasticity 16, 951-978 (2000). 
8. Eshelby, J. D.: The force on an elastic singularity. Phil. Trans. R. Soc. A 244, 87-112 (1951)

9. Evans, L. C.: Partial differential equations. Garaduate studies in mathematics, 19. Americam Math. Soc., Providence, Rhode Island (2010)

10. Falk, F.: Elastic phase transitions and nonconvex energy function. In: Free Boundary Problems: Theory and Applications, Pitman Research Notes in Mathematics Series 158, 49-50. Harlow, Longman (1990)

11. Ganghoffer, J. F., Plotnikov, P. I., Sokolowski, J.: Mathematical modeling of volumetric material growth in thermoelasticity. J. Elasticity 117, 111-138 (2014)

12. Ganghoffer, J. F., Plotnikov, P. I., Sokolowski, J.: Mathematical modeling of volumetric material growth. Archive of Applied Mechanics 84, 1357-1371 (2014)

13. Ganghoffer J.F., On Eshelby tensors in the context of open systems: application to volumetric growth. Int. J. Engng Sci. doi: 10.1016/j.ijengsci.2010.04.003.

14. Ganghoffer J.F., 2011. Mechanical modeling of growth considering domain variation - Part II: volumetric and surface growth involving Eshelby tensors. J. Mech. Phys. Solids, doi: 10.1016/j/jmps/2010.05.003.

15. Gilbarg, D., Trudinger, N.: Elliptic partial differential equations of second order. Second edition. Grundlehren der Mathematischen Wissenschaften 224 Springer-Verlag, Berlin ( 1983)

16. Jones G.W., Chapman S.J.: Modeling Growth in Biological materials. SIAM Review 54, 52-118 (2012)

17. Kuhl, E., Maas, R., Himpel, G., Menzel, A.: Computational modeling of arterial wall growth. Biomech. and Model. in Mechanobiol. 6, 321-331 (2007)

18. Menzel, A., Kuhl, E.: Frontiers in growth and remodeling. Mech. Res. Comm. 42, 1-14 (2012)

19. Menzel, A.: A fibre reorientation model for orthotropic multiplicative growth. Biomech. and Modeling in Mechanobiol. 6, 303-320 (2007)

20. Olsson, T., Klarbring, A.: Residual stresses in soft tissue as a consequence of growth and remodeling : application to an arterial geometry. Eur. J. Mech.A Solids 27, 959-974 (2008)

21. Pawłow, I., Żochowski, A.: Existence and uniqueness of solutions for a three -dimensional thermoelastic system. Dissert. Math. 406, 1-46 (2002)

22. Rodriguez, E., Hoger, A., McCulloch, A.: Stress-dependent finite growth law in soft elastic tissue. J. Biomech. 27, 455-467 (1994)

23. Rodriguez, J., Goicolea, J., Gabaldón, F.: 2007. A volumetric model for growth of arterial walls with arbitrary geometry and loads. J. Biomech. 40, 961-971 (2007)

24. Simon, J.: Compact sets in $L^{p}(0, T ; B)$, Annali di Matematica pura et applicata 146, 65-96 (1987)

25. Sprekels, J., Zheng, S.: Global Solutions to the Equations of a Ginsburg-Landau Theory for structural phase transitions in shape memory allows. Phys. D 39, 59-76 (1989)

26. Taber, L.: A model for aortic growth based on fluid shear and fiber stresses. J. Biomech. Eng. 120, 348-354 (1998)

27. Vignes, C., Papadopoulos, P.: Material growth in thermoelastic continua : Theory, algorithmics, and simulation. Computer Methods in Appl. Mech. and Eng. 199, 979-996 (2010) 